

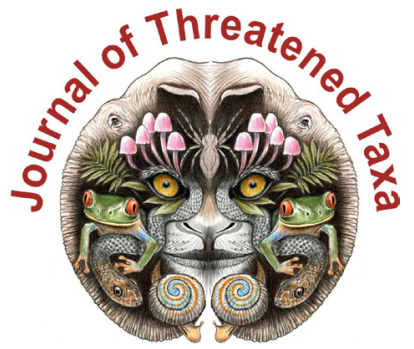

ISSN 0974-7907 (Online); ISSN $0974-7893$ (Print)

Publisher

Host

Wildlife Information Liaison Development Society

www.wild.zooreach.org

Zoo Outreach Organization www.zooreach.org

No. 12, Thiruvannamalai Nagar, Saravanampatti - Kalapatti Road, Saravanampatti, Coimbatore, Tamil Nadu 641035, India

Ph: +91 9385339863 | www.threatenedtaxa.org

Email: sanjay@threatenedtaxa.org

EDITORS

\section{Founder \& Chief Editor}

Dr. Sanjay Molur

Wildlife Information Liaison Development (WILD) Society \& Zoo Outreach Organization (ZOO),

12 Thiruvannamalai Nagar, Saravanampatti, Coimbatore, Tamil Nadu 641035, India

\section{Deputy Chief Editor}

Dr. Neelesh Dahanukar

Noida, Uttar Pradesh, India

\section{Managing Editor}

Mr. B. Ravichandran, WILD/ZOO, Coimbatore, India

\section{Associate Editors}

Dr. Mandar Paingankar, Government Science College Gadchiroli, Maharashtra 442605, India

Dr. Ulrike Streicher, Wildlife Veterinarian, Eugene, Oregon, USA

Ms. Priyanka Iyer, ZOO/WILD, Coimbatore, Tamil Nadu 641035, India

Dr. B.A. Daniel, ZOO/WILD, Coimbatore, Tamil Nadu 641035, India

\section{Editorial Board}

Dr. Russel Mittermeier

Executive Vice Chair, Conservation International, Arlington, Virginia 22202, USA

\section{Prof. Mewa Singh Ph.D., FASc, FNA, FNASc, FNAPsy}

Ramanna Fellow and Life-Long Distinguished Professor, Biopsychology Laboratory, and Institute of Excellence, University of Mysore, Mysuru, Karnataka 570006, India; Honorary Professor, Jawaharlal Nehru Centre for Advanced Scientific Research, Bangalore; and Adjunct Professor, National Institute of Advanced Studies, Bangalore

\section{Stephen D. Nash}

Scientific Illustrator, Conservation International, Dept. of Anatomical Sciences, Health Sciences Center, T-8, Room 045, Stony Brook University, Stony Brook, NY 11794-8081, USA

\section{Dr. Fred Pluthero}

Toronto, Canada

\section{Dr. Priya Davidar}

Sigur Nature Trust, Chadapatti, Mavinhalla PO, Nilgiris, Tamil Nadu 643223, India

\section{Dr. Martin Fisher}

Senior Associate Professor, Battcock Centre for Experimental Astrophysics, Cavendish

Laboratory, JJ Thomson Avenue, Cambridge CB3 OHE, UK

\section{Dr. John Fellowes}

Honorary Assistant Professor, The Kadoorie Institute, 8/F, T.T. Tsui Building, The University of Hong Kong, Pokfulam Road, Hong Kong

\section{Prof. Dr. Mirco Solé}

Universidade Estadual de Santa Cruz, Departamento de Ciências Biológicas, Vice-coordenado do Programa de Pós-Graduação em Zoologia, Rodovia Ilhéus/Itabuna, Km 16 (45662-000)

Salobrinho, Ilhéus - Bahia - Brasil

\section{Dr. Rajeev Raghavan}

Professor of Taxonomy, Kerala University of Fisheries \& Ocean Studies, Kochi, Kerala, India

\section{English Editors}

Mrs. Mira Bhojwani, Pune, India

Dr. Fred Pluthero, Toronto, Canad

Mr. P. Ilangovan, Chennai, India

Web Development

Mrs. Latha G. Ravikumar, ZOO/WILD, Coimbatore, India

\section{Typesetting}

Mr. Arul Jagadish, ZOO, Coimbatore, India

Mrs. Radhika, ZOO, Coimbatore, India

Mrs. Geetha, ZOO, Coimbatore India
Fundraising/Communications

Mrs. Payal B. Molur, Coimbatore, India

Subject Editors 2018-2020

Fungi

Dr. B. Shivaraju, Bengaluru, Karnataka, India

Dr. R.K. Verma, Tropical Forest Research Institute, Jabalpur, India

Dr. Vatsavaya S. Raju, Kakatiay University, Warangal, Andhra Pradesh, India

Dr. M. Krishnappa, Jnana Sahyadri, Kuvempu University, Shimoga, Karnataka, India

Dr. K.R. Sridhar, Mangalore University, Mangalagangotri, Mangalore, Karnataka, India

Dr. Gunjan Biswas, Vidyasagar University, Midnapore, West Bengal, India

\section{Plants}

Dr. G.P. Sinha, Botanical Survey of India, Allahabad, India

Dr. N.P. Balakrishnan, Ret. Joint Director, BSI, Coimbatore, India

Dr. Shonil Bhagwat, Open University and University of Oxford, UK

Prof. D.J. Bhat, Retd. Professor, Goa University, Goa, India

Dr. Ferdinando Boero, Università del Salento, Lecce, Italy

Dr. Dale R. Calder, Royal Ontaro Museum, Toronto, Ontario, Canada

Dr. Cleofas Cervancia, Univ. of Philippines Los Baños College Laguna, Philippines

Dr. F.B. Vincent Florens, University of Mauritius, Mauritius

Dr. Merlin Franco, Curtin University, Malaysia

Dr. V. Irudayaraj, St. Xavier's College, Palayamkottai, Tamil Nadu, India

Dr. B.S. Kholia, Botanical Survey of India, Gangtok, Sikkim, India

Dr. Pankaj Kumar, Kadoorie Farm and Botanic Garden Corporation, Hong Kong S.A.R., China

Dr. V. Sampath Kumar, Botanical Survey of India, Howrah, West Bengal, India

Dr. A.J. Solomon Raju, Andhra University, Visakhapatnam, India

Dr. Vijayasankar Raman, University of Mississippi, USA

Dr. B. Ravi Prasad Rao, Sri Krishnadevaraya University, Anantpur, India

Dr. K. Ravikumar, FRLHT, Bengaluru, Karnataka, India

Dr. Aparna Watve, Pune, Maharashtra, India

Dr. Qiang Liu, Xishuangbanna Tropical Botanical Garden, Yunnan, China

Dr. Noor Azhar Mohamed Shazili, Universiti Malaysia Terengganu, Kuala Terengganu, Malaysia

Dr. M.K. Vasudeva Rao, Shiv Ranjani Housing Society, Pune, Maharashtra, India

Prof. A.J. Solomon Raju, Andhra University, Visakhapatnam, India

Dr. Mandar Datar, Agharkar Research Institute, Pune, Maharashtra, India

Dr. M.K. Janarthanam, Goa University, Goa, India

Dr. K. Karthigeyan, Botanical Survey of India, India

Dr. Errol Vela, University of Montpellier, Montpellier, France

Dr. P. Lakshminarasimhan, Botanical Survey of India, Howrah, India

Dr. Larry R. Noblick, Montgomery Botanical Center, Miami, USA

Dr. K. Haridasan, Pallavur, Palakkad District, Kerala, India

Dr. Analinda Manila-Fajard, University of the Philippines Los Banos, Laguna, Philippines

Dr. P.A. Sinu, Central University of Kerala, Kasaragod, Kerala, India

Dr. Afroz Alam, Banasthali Vidyapith (accredited A grade by NAAC), Rajasthan, India

Dr. K.P. Rajesh, Zamorin's Guruvayurappan College, GA College PO, Kozhikode, Kerala, India

Dr. David E. Boufford, Harvard University Herbaria, Cambridge, MA 02138-2020, USA

Dr. Ritesh Kumar Choudhary, Agharkar Research Institute, Pune, Maharashtra, India

Dr. Navendu Page, Wildlife Institute of India, Chandrabani, Dehradun, Uttarakhand, India

\section{Invertebrates}

Dr. R.K. Avasthi, Rohtak University, Haryana, India

Dr. D.B. Bastawade, Maharashtra, India

Dr. Partha Pratim Bhattacharjee, Tripura University, Suryamaninagar, India

Dr. Kailash Chandra, Zoological Survey of India, Jabalpur, Madhya Pradesh, India

Dr. Ansie Dippenaar-Schoeman, University of Pretoria, Queenswood, South Africa

Dr. Rory Dow, National Museum of natural History Naturalis, The Netherlands

Dr. Brian Fisher, California Academy of Sciences, USA

Dr. Richard Gallon, llandudno, North Wales, LL30 1UP

Dr. Hemant V. Ghate, Modern College, Pune, India

Dr. M. Monwar Hossain, Jahangirnagar University, Dhaka, Bangladesh

Mr. Jatishwor Singh Irungbam, Biology Centre CAS, Branišovská, Czech Republic.

Dr. Ian J. Kitching, Natural History Museum, Cromwell Road, UK

Dr. George Mathew, Kerala Forest Research Institute, Peechi, India

For Focus, Scope, Aims, and Policies, visit https://threatenedtaxa.org/index.php/JoTT/aims_scope
For Article Submission Guidelines, visit https://threatenedtaxa.org/index.php/JoTT/about/submissions
For Policies against Scientific Misconduct, visit https://threatenedtaxa.org/index.php/JoTT/policies_various

continued on the back inside cover 


\title{
An inventory of geometrid moths (Lepidoptera: Geometroidea: Geometridae) of Kalakad-Mundanthurai Tiger Reserve, India
}

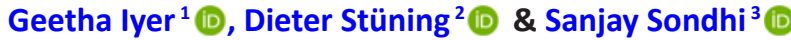

\author{
${ }^{1}$ 11/49, Teppakulam street, Suchindrum-629704, Kanyakumari District, Tamil Nadu, India. \\ ${ }^{2}$ Zoological Research Museum Alexander Koenig, Adenauerallee 160, 53113 Bonn, Germany. \\ ${ }^{3}$ Titli Trust, 49, Rajpur Road Enclave, Dhoran Khas, near IT Park, PO Gujrada, Dehradun, Uttarakhand 248013, India. \\ ${ }^{3}$ Indian Foundation for Butterflies. C-703, Alpine Pyramid, Rajiv Gandhi Nagar, Bengaluru, Karnataka 560097, India. \\ ${ }^{1}$ scopsowl@gmail.com (corresponding author), ${ }^{2}$ d.stuening@leibniz-zfmk.de, ${ }^{3}$ sanjay.sondhi1@gmail.com
}

\begin{abstract}
The geometrid moths of Kalakad-Mundanthurai Tiger reserve were studied during the years 2012 to 2016 . Since collection of specimens was not permitted, only field notes, accompanied by photo documentation was undertaken. Two-hundred-and-sixty geometrid moths identified to various hierarchical levels of taxa and one new genus for southern India, are reported.
\end{abstract}

Keywords: Agasthyamalai, biodiversity, Heterocera, KMTR, moth diversity, southern Western Ghats, Tamil Nadu.

Abbreviations: FW-Forewing | HW-Hindwing | KMTR-Kalakad-Mundanthurai Tiger Reserve, Tamil Nadu | KKWS-Kanyakumari Wildlife Sanctuary, Tamil Nadu | MoB-Moths of Borneo | UN-Underside | UP-Upperside | ZFMK-Zoological Research Museum Alexander Koenig, Bonn, Germany.

Citation: Iyer, G., D. Stüning \& S. Sondhi (2021). An inventory of geometrid moths (Lepidoptera: Geometroidea: Geometridae) of Kalakad-Mundanthurai Tiger Reserve, India. Journal of Threatened Taxa 13(13): 19887-19920. https://doi.org/10.11609/jott.7105.13.13.19887-19920

Copyright: (c) Iyer et al. 2021. Creative Commons Attribution 4.0 International License. JoTT allows unrestricted use, reproduction, and distribution of this article in any medium by providing adequate credit to the author(s) and the source of publication.

Funding: Self funded.

Competing interests: The authors declare no competing interests.

Author details: GEETHA IYER is an independent educational consultant and author who writes for and trains teachers to help bring India's biodiversity into biology and environmental science classes. She is particularly interested in the moths of the Southern Western Ghats which she has studied for many years at KMTR and KKWS. She has authored two books, authored and edited school text books, published scientific papers, and writes popular articles on moths and other insects. DIETER STÜNING is a German Geometridae specialist, who was Head of the Lepidoptera \& Trichoptera Section of the Zoological Research Museum Alexander Koenig at Bonn for more than 30 years. Since retiring in 2014, he is working as a scientific associate, continuing to study Palearctic and Asian Geometridae, especially the taxonomy, systematics and phylogeny of subfamily Ennominae. He has described numerous new taxa of species and genera and also co-authored the description of a new moth-family. SANJAY SONDHI is a Dehradun-based naturalist. An engineering graduate from IIT-Kanpur, in 2009 he set up TITLI TRUST (wWW. titlitrust.org), a nature conservation not-for-profit organisation, which is devoted to studying and protecting India's lesser known flora and fauna. He has authored numerous books and technical papers on amphibians and reptiles, birds, butterflies and moths and other Indian wildlife.

Author contributions: GI-conceived and designed the study, surveyed and photographed the moths, prepared the species list, and wrote the manuscript. DS - supported species identifications with the help of literature and comparison with specimens of the ZFMK collection, and editing of the manuscript. SSsupported species identifications and editing of the manuscript during its various drafts.All three authors approve the final version.

Acknowledgements: This work would not have been possible without the permissions and assistance extended by forest personnel of different cadres. GI thanks the many Field directors, Deputy directors, foresters, and rangers of KMTR - past and present- for permissions to survey moths and the guards, watchers and anti-poaching watchers who accompanied her on trails, and provided her with support and help. GI also thanks the former DFO Mr Ritto Cyriac, rangers, forester and anti-poaching watchers of Kanyakumari Wildlife Sanctuary, and Mr Thomas Devasahayam of Maramalai for the assistance and help given. Thanks are due to ecologist Ms B. Smitha for her wide-ranging assistance during the surveys. We thank Rikio Sato from Japan who helped in identifying some of the geometrids of the tribe Boarmiini. GI thanks Axel Hausmann and Pasi Sihvonen for sharing the relevant and most recent publications, as well as answering and clarifying the innumerable number of queries put to them. She thanks lan Kitching, Jeremy D. Holloway Roger Clive Kendrick, Navneet Singh, Pritha Dey, Rahul Joshi, H. Sankararaman and Balakrishna Valappil for their help in clarifying doubts or sourcing papers and/or in helping to identify geometrids. GI thanks S. Thalavaipandi, researcher from ATREE, Bangalore, for sharing the images of geometrids from Upper Kothayar.
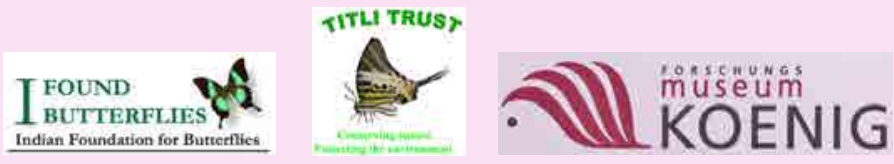


\section{INTRODUCTION}

Kalakad-Mundanthurai Tiger Reserve (KMTR) is located at the southern Western Ghats, in the Agasthyamalai range, approximately between $8.416 \mathrm{~N}$ and $8.883 \mathrm{~N}$ latitude and 77.166E and 77.583E longitude. It falls within Kanyakumari and Tirunelveli districts of the State of Tamil Nadu, India and is part of the Agasthyamalai Biosphere Reserve. With a core area of $895.39 \mathrm{~km}^{2}$, KMTR was established as a tiger reserve in 1988 through the merger of Kalakad Wildlife Sanctuary, Mundanthurai Tiger Reserve, and parts of Veerapuli and Kilamalai reserve forests, from Kanyakumari district. The Nellai Wildlife Sanctuary, encompassing an area of 35.9 $\mathrm{km}^{2}$, in the north and $201.36 \mathrm{~km}^{2}$ of the Kanyakumari Wildlife Sanctuary towards the south form the buffer area of the reserve. The elevation ranges 100-1,880 m. Thus, a gradient of vegetation ranging from dry scrub to dense evergreen forest is found here. The reserve receives close to $3,000 \mathrm{~mm}$ of rainfall from both the south-west and north-east monsoons. It serves as a catchment area for no less than 14 rivers and streams. For this reason, it is sometimes referred to as a river sanctuary. The Agasthyamalai hills, which includes the core area of KMTR, are an important biogeographical 'hot spot' within the Western Ghats and a region of endemism in India (UNESCO 2016).

Being a tiger reserve, the biodiversity studies in this region have largely been focussed on mammals, herpetofauna, and plants for many years. Recent studies on invertebrates have been mostly on spiders or butterflies, and moths have not received any attention. This study is an attempt to enumerate the geometrid moths of this reserve. It is the first-of-its-kind study of moth diversity for this region, covering all habitats of the reserve, through seasons over multiple years.

Geometridae (Stephens, 1829) constitute the second largest family of moths in India, with at least 2,043 species listed so far (Kirti et al. 2019; Sondhi et al. 2020; Dey et al. 2021) from India. A large number of these slender moths, are mostly nocturnal, but day flying and crepuscular species are not uncommon. Some geometrids are strongly haired but most are examples of least-haired moths. In the forests of KMTR, we have observed them arrive in large numbers when the mist begins to set in. Protective colouration and camouflage were observed. Polymorphism was noticeable in many species. This paper outlines through photographic records, the diversity of geometrid moths arising out of a five-year survey in KMTR.

Geometrids have been reported from other parts of
Western Ghats too: 77 species from Silent Valley National Park by Mathew \& Rahamathulla (1995); six species by Bharmal (2015) from Amboli, Maharashtra and four species by Mishra et al. (2016) from Kodagu. From Kerala there are records of 47 species from ShendurneyPonmudi by Sondhi et al. (2018). Elenchezhian et al. (2014) reported 28 species from Maruthamalai hills and Goyal (2010) described 19 species for his PhD. The last two are the only recently published records from Tamil Nadu and even these studies were restricted to Madurai and Nilgiri districts.

A literature survey of the older Indian records in Moore $(1884-7,1889)$ or Hampson $(1891,1893)$ finds no mention of moths from Kalakad or Mundanthurai. A few scattered records of geometrids from 'Travancore State' are available. The State of Travancore was dissolved soon after India attained independence and the places under its jurisdiction were distributed between the present States of Kerala and Tamil Nadu. Kanyakumari, from the erstwhile southern part of Travancore State, thus became a part of Tamil Nadu. Published records of geometrid moths from the southern part of the erstwhile Travancore State are not available. Hence it is safe to assume that there are no historical records of moths from this region.

Some moth species have been recorded from KMTR in recent years by other researchers. Ron Brechlin described a sphingid, Ambulyx sinjaevi (Brechlin 1998), and a saturniid, Loepa schint/meisteri (Brechlin 2000); Stauropus thiaucourti, a notodontid moth, was reported by Schintlmeister (2003); a cossid Phragmacossia brahmana, by Yakovlev (2009) and three geometrid species, Racotis keralaria, Ophthalmitis kalakadaria, and Hypomecis tamilensis, were described by Sato (2004, 2014, 2016). All these species were recorded from Manjolai (8.250N and 77.433E), a very small area of a few sq. km. All of them were new to science. There are no other published records of geometrids from KMTR. Thus, most of the moths presented are previously unrecorded, several unidentified and still to be described or species that are range extensions. One of them is a new record for southern India. Hence the moth inventory in this paper is the first attempt to generate a comprehensive list of geometrid moths found in this Tiger Reserve.

\section{MATERIALS AND METHODS}

\section{Study sites}

The moths listed in this paper were extensively surveyed from within the core area of KMTR during the 
years 2012 to 2015, covering most of the months except July and August, primarily due to lack of access during the monsoons. Moths were surveyed very briefly in the year 2016 and again, extensively, in the year 2019. The sites of study were Talayanai, Sengeltheri, Kuthiraivetti, Upper Kothayar, Mundanthurai, and Kannikatti. The list also includes geometrids from one of the buffer zones of KMTR, namely Kanyakumari Wildlife Sanctuary (KKWS), from sites located at Maramalai and Kalikesam (Table 1 \& Figure 1-3. Locations and sites of study). Permission for collection was not available from the core or the buffer area of KMTR as the sites are within a legally protected area.

Some moths presented are not part of the planned survey, but were opportunistic observations during the years 2016, 2018, and early 2019. All sites being within the core regions of the Tiger Reserve, surveys were dependent on permissions from forest department, weather conditions, availability of accommodation, and finance. The entire study was self-funded by the first author.

\section{Survey methods}

Moths were surveyed using a light trap consisting of a $160 \mathrm{~W}$ mercury vapour bulb hung above a white cotton sheet measuring $3 \times 5$ feet $(0.9144 \times 1.524$ meters), stretched between either two posts, trees, window bars, or sometimes, nails on a wall. Wherever electricity was not available (Sengeltheri and Kannikatti), a kerosene or petrol-powered Honda generator was used. The white cloth screen was illuminated starting at $1800 \mathrm{~h}$ or 1830 $\mathrm{h}$, depending upon sunset, until $0130 \mathrm{~h}$. Where mains electricity was available, the MV bulb was kept on till 0400 h. Diurnal activity of moths was noted at all locations.

Digital photographs were taken using a Panasonic FZ 200 and a Panasonic FZ 35 with a Lumix lens. Field notes were recorded for morphological details and of features that could not be captured through photography.

\section{Methods for identification}

The Tamil Nadu Forest Department discourages collection of specimens, even for research. This is one of the reasons for the poor records of moths and other insects from this region. Despite providing photographic evidence for new range and species records, permission to collect voucher specimens was not granted. Consequently, field notes and digital photography were the only methods available for assessing the diversity. The notes, photographs, comparisons (of photos) with museum specimens from ZFMK, Bonn, published papers,
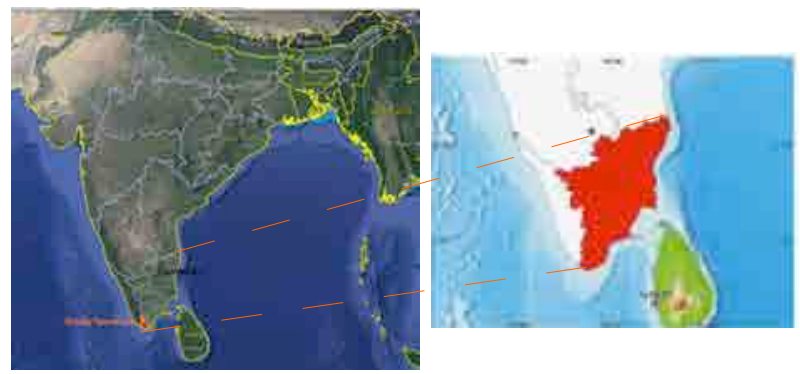

Figure 1. Map of India showing the state of Tamil Nadu.

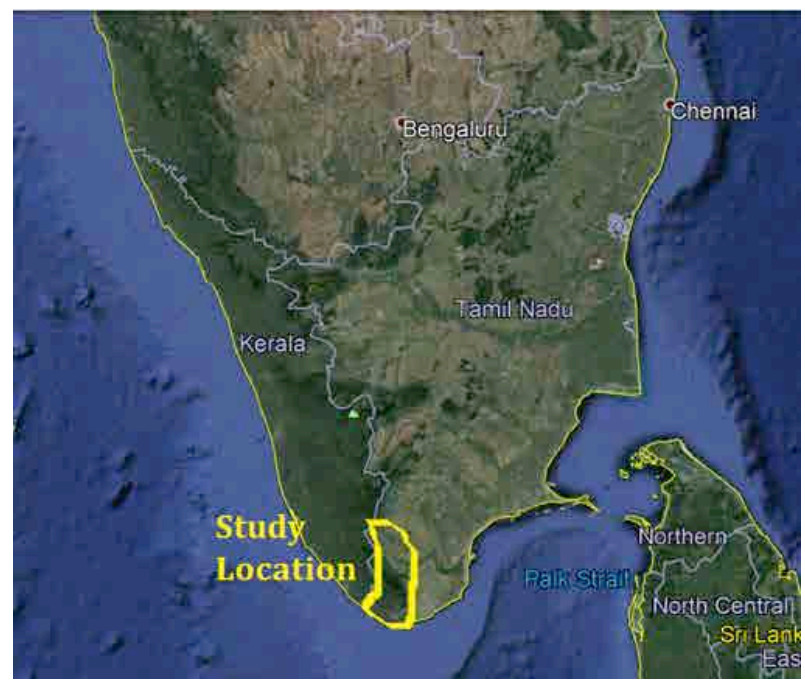

Figure 2. Map of India showing the general study location in the state of Tamil Nadu

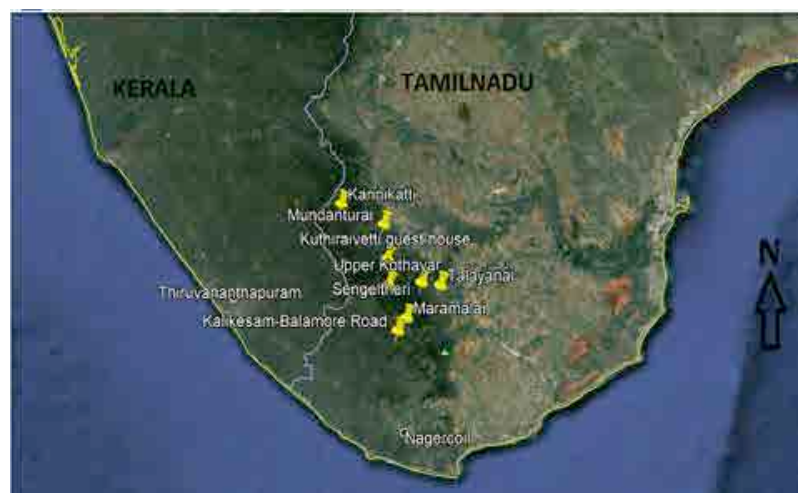

Figure 3. Sites of study in KMTR.

discussions with experts and researchers, and historical records have been the methods used for identification.

Walker (1860-62), Moore (1879, 1884-87), Swinhoe (1890), Hampson (1891, 1893, 1895), Rothschild (1894), Warren (1894, 1897), Prout (1912, 1917, 1920-41), Inoue (1953, 1972), Barlow (1982), Holloway (1983-2011), Butler (1886, 1889), and Scoble et al. (1999) were the 
Table 1. Location of study sites along with elevation, habitat, and timeline.

\begin{tabular}{|c|l|c|c|c|l|l|}
\hline & Place & Latitude & Longitude & $\begin{array}{c}\text { Elevation } \\
\text { in metres }\end{array}$ & Habitat type \\
\hline 1 & Talayanai & $8.526^{\circ} \mathrm{N}$ & $77.502^{\circ} \mathrm{E}$ & 224 & Riparian \\
\hline 2 & Sengeltheri & $8.534^{\circ} \mathrm{N}$ & $77.45^{\circ} \mathrm{E}$ & 984 & Semi-evergreen & 2012,2015 \\
\hline 3 & Kuthiraivetti & $8.5931^{\circ} \mathrm{N}$ & $77.352^{\circ} \mathrm{E}$ & 1,146 & Moist deciduous & 2015 \\
\hline 4 & Upper Kothayar & $8.527^{\circ} \mathrm{N}$ & $77.359^{\circ} \mathrm{E}-14,2019$ & $\begin{array}{l}\text { Evergreen and } \\
\text { montane }\end{array}$ \\
\hline 5 & Mundanthurai & $8.6563^{\circ} \mathrm{N}$ & $77.3332^{\circ} \mathrm{E}$ & $2012-14,2018,2019$ \\
\hline 6 & Kannikatti & $8.732^{\circ} \mathrm{N}$ & $77.226^{\circ} \mathrm{E}$ & 250 & $\begin{array}{l}\text { Riverine and dry } \\
\text { deciduous }\end{array}$ \\
\hline 7 & Maramalai & $8.45^{\circ} \mathrm{N}$ & $\begin{array}{l}\text { Wet evergreen and } \\
\text { riparian }\end{array}$ & 2012,2015 \\
\hline 8 & Kalikesam & $8.41^{\circ} \mathrm{N}$ & $77.4^{\circ} \mathrm{E}$ & $\begin{array}{l}\text { Mixed forest and } \\
\text { estates }\end{array}$ & 2012,2015 \\
\hline
\end{tabular}

prime sources of reference. Other than these sources, several research papers and books were also consulted. The second author referred extensively with the museum specimens at ZFMK, to arrive at identifications, and the third author used Moths of India website for the same. The classification in the paper, unless specified otherwise, follows that of Murillo-Ramos et al. (2019).

It is an established fact that without a specimen in hand, identification to the level of species is an extremely difficult task for many cryptic moth species. Hence, without specimens and therefore absence of genitalia information, it was not possible to identify all geometrids to the species level. Identifications, in such cases, have been limited to the level of subfamily, tribe or genus. For some individuals, where records or field data were insufficient, we have suggested provisional identifications. From our investigations, we believe that some of these could well be either new species to science or range extensions.

As this was the first comprehensive survey undertaken in this Tiger Reserve, most of the moths were first records for KMTR. They were also first records for the districts of Kanyakumari and Tirunelveli in the state of Tamil Nadu.

\section{Findings from the study}

Three-hundred-and-ninety geometrids belonging to five subfamilies were investigated. Only 260 are presented in this paper with identities at different levels. Six taxa were identified to the level of subfamily and six to the level of tribe. While 98 taxa were identified to genus level, 160 taxa, which included males, females as well as morphs, were identified to the level of species. Thirtyfour taxa are reported with provisional identifications using Open Nomenclature qualifiers (Box 1). They have been identified to the nearest recognisable species for
Box 1. Open Nomenclature qualifiers (ON)

Abbreviations used to indicate a taxon's provisional status of identification are termed as Open Nomenclature qualifiers. These qualifiers are used by researchers from different disciplines with some variations in interpretations. The qualifiers we have used for provisional identifications in this paper are 'cf.' and ' $n r$ '. These terms have been used with the following interpretations:

cf. This is short for the Latin word confero/conferatur, meaning, 'to compare with'. We have used it to indicate that the species identification is provisional but is likely to be conclusive when we have more data, when it is further compared or crosschecked with reference materials, or discussed with a specialist of the taxon. The degree of uncertainty is less when compared with the term 'nr'.

$\mathbf{n r}$ This is short for 'near'. This qualifier has been used when the moth bears some similarities or resemblances to a known species. It appears closely related but is not identical to it.

comparison, or to a possible species close to them for further study.

Overall, 98 genera and 108 species have been identified. Polymorphism was quite marked in species from subfamilies Ennominae, Larentiinae, and Sterrhinae. Other than Abraxas leucostola argyrosticta Hampson, 1893, Hypomecis tamilensis Sato, 2016, Luxiaria hypaphanes Hampson, 1891, Ophthalmitis kalakadaria Sato, 2014, and Racotis keralaria Sato, 2004, all the moths presented in this paper (Table 2) are new records for KMTR and, Kanyakumari and Tirunelveli districts of Tamil Nadu, with Acanthovalva Krüger, 2001, a newly recorded genus for southern India.

Investigations have pointed to the possibility of some new species among the many tentatively identified or unidentified moth records. We hope this baseline survey report will assist in obtaining permission to collect and thus initiate a more detailed study of moths in this region. 
Table 2. Summary of subfamilies, genera and species presented.

\begin{tabular}{|c|l|c|c|c|c|}
\hline & Subfamily & Genera & $\begin{array}{c}\text { Species with } \\
\text { confirmed } \\
\text { identity }\end{array}$ & $\begin{array}{c}\text { Species with } \\
\text { conditional } \\
\text { identity }\end{array}$ & $\begin{array}{c}\text { Species not } \\
\text { identified }\end{array}$ \\
\hline 1 & $\begin{array}{l}\text { Sterrhinae } \\
\text { Meyrick, 1892 }\end{array}$ & 12 & 13 & 5 & 3 \\
\hline 2 & $\begin{array}{l}\text { Larentiinae } \\
\text { Duponchel, 1845 }\end{array}$ & 13 & 9 & 2 & 3 \\
\hline 3 & $\begin{array}{l}\text { Geometrinae } \\
\text { Stephens, 1829 }\end{array}$ & 23 & 24 & 7 & 3 \\
\hline 4 & $\begin{array}{l}\text { Desmobathrinae } \\
\text { Meyrick, 1886 }\end{array}$ & 3 & 3 & 0 & 0 \\
\hline 5 & $\begin{array}{l}\text { Ennominae } \\
\text { Duponchel, 1845 }\end{array}$ & 47 & 59 & 20 & 11 \\
\hline
\end{tabular}

\section{Taxon notes}

Details of select genera/species are shared below. The taxon order followed is as given in The Forum Herbulot World List of Family Group Names in GeometridaeForum Herbulot (2003, updated 2007).

\section{Subfamily Sterrhinae Meyrick, 1892}

Plates 1-2 (5-36)

The classification of moths of this subfamily (Table 3 ) follows the most recent revision by Sihvonen et al. (2020). Of the 34 moths observed, 31 individuals from seven tribes were identified either to the genera or species level. Three individuals could not be identified further. Sihvonen et al. (2020) mention in their paper that a large number of species of this cosmopolitan family, comprising of nearly 3,000 moth-species worldwide, fall under the genera Idaea Treitschke, 1825 or Scopula Schrank, 1802. This abundance was reflected in our survey too.

\section{Idaea Treitschke, 1825}

Plate 2 (23-30)

Idaea Treitschke, 1825 is a genus with hundreds of small and very similar species which are difficult to identify even with voucher specimens in hand. In their absence, we have relied on published records and museum specimens. We report only three indivduals of Idaea (out of the 10 individuals), whose wing patterns were unique enough to be identified to species level. While researching to confirm Idaea gemmaria Hampson, 1896, (Plate 2(23)), we came across Holloway's statement in Moths of Borneo (Holloway 1997) about the taxon "I. gemmataria", Hampson (Sri Lanka). On contacting Dr Holloway (pers. comm., 21.ix.2020), he clarified that, "it was indeed an error arising at some stage between his reading the handwritten name in the museum collection and the final appearance of his text in print! The correct name of the species described by Hampson was indeed I. gemmaria". This has helped confirm the identity of the species.

The next most common species were from the genus Scopula Schrank, 1802 (Plates 1-2 (15-19)). Out of five individuals observed, two have been identified to the species level.

The key given by Xue et al. (2018) for the pattern of ocellus in the wings, descriptions and comparison with specimens in the ZFMK ollection, were used to identify Problepsis apollinaria Guenée, [1858] and P. deliaria Guenée, [1858] (Plate 1 (13-14)). All of the Sterrhinae listed are new records for KMTR.

\section{Subfamily Larentiinae Duponchel, 1845}

Plates 3-4 (37-60)

This is the second largest subfamily amongst Geometridae. Worldwide, 6,200 species (Õunap et al. 2016) have been described so far. We have recorded 24 moths from six tribes. Only nine of them could be identified to the species level. Fourteen larentiines reported belong to the tribe Eupitheciini Tutt, 1896. Of these, the genera Collix Guenée, [1858] and Eois Hübner, 1818 were the most represented. Three individuals from tribe Eupitheciini could not be identified even to the genus level. All the larentiine moths listed are new records for this region. We also believe that there are possibilities of new eupitheciine species from this region.

\section{Subfamily Geometrinae Stephens, 1829}

Plates 4-6 (61-101)

A large number of moths of this subfamily being green in colour, are often referred to as emerald moths. They were seen in large numbers at KMTR. However, the species diversity did not match individual abundance. According to Plotkin \& Kawahara (2020), the current checklist of Geometrinae worldwide stands at 2,642 species. We recorded 41 different individuals from which 24 species from 23 genera were identified. Three moths remained unidentified while the remaining were identified to the level of genera or provisionally to the nearest species.

\section{Tribe Comibaenini Inoue, 1961}

Plate 4 (66-74)

\section{Comibaena Hübner, [1823] 1816}

Plate 4 (66-72)

We report four species and one individual referred for comparison to the nearest species. Comibaena integranota Hampson, 1893 and Comibaena attenuata Warren, 1896, are differentiated based on the patches 
seen in the tornal region of forewing and apex of the hind wing. In attenuata, the forewing patch has an irregular projection anteriorly that is separated from the margin. Warren (1896), while describing attenuata under the older synonym Probolosceles attenuata Warren, 1896, has pointed out that some features of attenuata have been mistakenly attributed to the female of integranota by Hampson (1893).

\section{Protuliocnemis cf. biplagiata (Moore, 1887)}

Plate 4 (73)

Protuliocnemis biplagiata (Moore, [1887]) and $P$. castalaria (Oberthür, 1916), are similar in fasciae and genitalia. The number of spurs found in the hind tibia (Holloway 1996) is the only feature to distinguish the two species. Where as $P$. biplagata has four spurs in the hind tibia, P. castalaria has only two ((Prout 1933, GrossSchmett. Erde 12: 88) in Holloway, 1996). Protuliocnemis biplagiata has been reported from Sri Lanka and, northern \& northeastern of India and P. castalaria only from Khasi hills. This species of Protuliocnemis was frequently observed in both the core and the buffer zone of the reserve from the month of October to March. As the data of hind tibia spurs is not available and based on its presence in Sri Lanka, we report this individual provisionally as $P$. cf. biplagiata.

\section{Tribe Hemitheini Bruand, 1846}

Plate 5 (77-91)

We report 15 individuals belonging to 11 genera, of which six have been identified to the level of species; four are reported with provisional species identity and four are identified to the genus level. A few are detailed below.

\section{Pelagodes Holloway, 1996}

Plate 5 (83)

Moths of this genus were quite abundant in higher altitudes and found at all study sites except Talayani. The genus Pelagodes Holloway, 1996, was identified and differentiated from the closely resembling Thalassodes Guenée, [1858] based on the hindwing architecture as given by Han \& Xue (2011). They report that the outer margin of the hindwing is strongly angled in Thalassodes but not so in Pelagodes. Species level identification of Pelagodes was not attempted in the absence of genitalia details. The presence of Thalassodes cannot be ruled out as several individuals remained unidentified. Lack of specimens in hand was a constraint for further investigation.

\section{Jodis Hübner, 1823}

Plate 5 (87-89)

Three species of Jodis were observed. One has been identified to species level. One is provisionally identified to species level while the third could not be identified beyond the level of genus.

\section{Jodis pallescens (Hampson, 1891)}

Plate 5 (88)

Hampson (1893) has described this taxon from Nilgiris as Thalera pallescens and the description matches the species reported here. Prout (1934) reported it from Sri Lanka and described J. pallescens as a distinct species due to the sharp contrast seen between the green and the white areas. This was quite evident in the moths we observed and the details match the field notes.

\section{Jodis nr. undularia (Hampson, 1891)}

Plate 5 (87)

Hampson (1893) described it from Nilgiris, and misidentified it as Thalera caudularia. It is, according to Prout (1934), widely distributed in India. A yellowishgreen moth, it shows the features matching the description given by Hampson. The vertex of the head is white. The whitish antemedial and postmedial lines are dentate, excurved between veins 2 and 4, giving it, at first glance, a greyish-green appearance. The underside is white with shades of greyish-white. As Prout (1934) pointed, the tail of the hindwing is sharp. We, therefore, suggest the species identity tentatively, to facilitate further exploration.

\section{Tribe Nemoriini Gumppenberg, 1887 \\ Eucylodes albisparsa (Walker, 1861)}

Plate 5 (92)

Ban et al. (2018) placed Eucylodes in the tribe Nemoriini. However, its tribal position is reported as uncertain in the research paper by Murillo-Ramos et al. (2019). The paper also does not assign it to any tribe. We have retained the species in Tribe Nemoriini, after consulting $\mathrm{Dr}$ Hausmann (Hausmann, pers. comm. 01.vii.2020) who is one of the co-authors of the 2019 paper.

Eucyclodes albisparsa and E. divapala (Walker, 1861), are similar species, which fly in southern India. They are best separated by genitalic features. However, Barlow (1982) reports that the latter species found in southern India is more uniformly green and less contrasting than divapala. This matches the features in the individual observed by us. Hence, we report this moth as belonging to Eucyclodes albisparsa complex. 


\section{Subfamily Desmobathrinae Meyrick, 1886}

Plate 6 (102-105)

Four individuals from three genera are presented here. Derambila fragilis (Butler, 1881), Noreia ajaia Walker, 1859, Ozola microniaria Walker, 1862 and the fourth moth belonging to the genus Ozola Walker, [1861] could not be identified to species level.

\section{Derambila Walker, [1863] 1862}

Plate 6 (102)

Members of Derambila are small, slender, translucent white moths. Derambila saponaria (Guenée, [1858]) has been recorded from Travancore and Sri Lanka. Hampson (1895) records it as Rambara saponaria Guenée from Travancore. Rambara Moore, [1887] is currently treated as a junior synonym of Derambila (Scoble et al. 1999). Derambila fragilis (Butler, 1881), (Taiwan), described erroneously in the genus Zanclopteryx Herrich-Schäffer, [1855], is another similar species. Zanclopteryx is a genus with exclusively New World species. Prout (1921) notes that in fragilis, the antemedian lines are very few and mostly dissolved into spots. Holloway (1996), states that $D$. fragilis is also found in India and records that the wings are relatively slightly marked and lack the brown suffusion, seen in other species. In D. saponaria, the brown transverse markings are well expressed whereas in $D$. fragilis, they are present as dots. Based on the markings on the wings and the distribution given by Hampson \& Moore, we report this species as Derambila fragilis.

\section{Subfamily Ennominae Duponchel, 1845}

Plates 6-13 (106-274)

With no anatomical details being available, members of the largest geometrid subfamily were the most challenging to identify from external morphological only. Along with historical records and published papers, the collections from ZFMK were most useful for identification. Polymorphism was quite vivid in several genera which added to the complexity. Explanations of our conclusions for select taxa are given below.

\section{Tribe Abraxini Warren, 1893}

\section{Abraxas Leach, [1815]}

Plates 6-7 (106-123)

Specimens of the genus Abraxas were found in abundant numbers in Upper Kothayar, Kuthiraivetti, Sengeltheri, and Maramalai. Materials from Kalakad, Nilgiris, and Sri Lanka available in the collections at ZFMK were compared along with descriptions and figures published by Hampson (1891, 1893, 1907),
Swinhoe (1890, 1891), Walker (1862), Warren (1894, 1898), and Prout (1925) for identification. Of the 15 individuals presented here, three are identified to the level of species (Plates 6-13 (106-109)). The rest of the unidentified individuals are in varying shades of grey and bluish-grey, with or without irregular yellow spots that ranged from many to none on the upperside. The underside of these specimens varied from being grey to grey with more or less irregular yellow spots and to one with a completely yellow underside with dark fasciae (Plate 7 (115)), the latter certainly a distinct species, the former probably variations of one species. All these species had yellow heads. The antennae were completely black or grey. The thorax showed varying degrees of greyness. Their legs were grey with yellow coxa. Large black spots on the upper side of the abdominal segment, and smaller ones on the sides and undersides were present. Based on these differences in patterns and colours on the upper and underside, the grey Abraxas moths and that with grey upper side and yellow underside have been categorised tentatively as different species. We cannot rule out the fact that some of them are probably just forms of two or more species. Hence collection is needed to investigate further and get greater clarity of this group of moths that were present during all seasons.

Another unidentified Abraxas was completely yellow on upper and underside, with grey postmedial fasciae and spots (Plate 7 (121-123)) which bore no resemblance to the two yellow Abraxas hitherto described from southern India, namely, A. Iuteoaria Swinhoe, 1890 and A. germana Swinhoe, 1891. Both have been described from Nilgiri Hills, but were not spotted at KMTR during our surveys. The second author who is familiar with the southern Indian Abraxas species found that the grey and the yellow series of Abraxas we have presented does not resemble any existing species. We therefore report them as unidentified species of Abraxas that need further investigation.

About 20 species of the genus Abraxas have been described from southern India and Sri Lanka at the end of the $19^{\text {th }}$ century and the beginning of the $20^{\text {th }}$ century. Most of them are endemic to the Western Ghats and do not look (Plates 6-13 (110-120)) like typical Abraxas, as known from examples seen in the Himalaya or Chinese mountainous regions. Only A. leucostola Hampson, 1893, described from Sri Lanka and later described from southern India by Hampson (1907) as Abraxas argyrosticta (which is at present treated as subspecies of leucostola), resembles the typical Abraxas. A. fasciaria (Guérin-Méneville, 1843), A. poliostrota Hampson, 1907, 
Table 3. Checklist of geometrids of Kalakad-Mundanthurai Tiger Reserve.

\begin{tabular}{|c|c|c|c|c|c|}
\hline & S. Family/ Genus & Tribe/ Species & $\begin{array}{l}\text { Author \& Year: S. Family/ } \\
\text { Tribe/ Genus/ Species }\end{array}$ & Location & Month and Year of survey \\
\hline & $\begin{array}{l}\text { Sterrhinae } \\
\text { Meyrick,1892 }\end{array}$ & Cosymbiini & Prout, 1911 & & \\
\hline 1 & Chrysocraspeda & sp.1 & Swinhoe, 1893 & Upper Kothayar & Mar 2014 \\
\hline 2 & Chrysocraspeda & sp. 2 & Swinhoe, 1893 & Kannikatti, Kuthiraivetti & Feb 2013, Mar 2014 \\
\hline \multirow[t]{2}{*}{3} & Perixera & insitiva & (Prout, 1920) & Kuthiraivetti & Dec 2012 \\
\hline & & Cyllopodini & Kirby, 1892 & & \\
\hline \multirow[t]{2}{*}{4} & Organopoda & sp. & Hampson, 1893 & Upper Kothayar & Oct 2018 \\
\hline & & Lissoblemmini & Sihvonen \& Staude, 2020 & & \\
\hline 5 & Lissoblemma & lunuliferata & (Walker, [1863]) & Upper Kothayar & Oct 2018 \\
\hline \multirow[t]{2}{*}{6} & Craspediopsis & sp. & Warren, 1895 & Talayanai & Feb 2015 \\
\hline & & Rhodometrini & Agenjo, 1952 & & \\
\hline 7 & Traminda & aventiaria & (Guenée, [1858]) & Kuthiraivetti & Dec 2012 \\
\hline \multirow[t]{2}{*}{8} & Traminda & $\begin{array}{l}\text { mundissima - } \\
3 \text { forms }\end{array}$ & (Walker,1861) & $\begin{array}{l}\text { Mundanthurai, Talayanai, } \\
\text { Kuthiraivetti }\end{array}$ & $\begin{array}{l}\text { Mar 2012, Oct 2012, } \\
\text { Feb 2015, Dec } 2012\end{array}$ \\
\hline & & Scopulini & Duponchel, 1845 & & \\
\hline 9 & Problepsis & deliaria & (Guenée, [1858]) & $\begin{array}{l}\text { Kuthiraivetti, Sengeltheri, } \\
\text { Upper Kothayar, } \\
\text { Maramalai }\end{array}$ & $\begin{array}{l}\text { Dec 2011, 2012, 2019, } \\
\text { Oct 2012, June 2013, } \\
\text { Feb } 2012\end{array}$ \\
\hline 10 & Problepsis & appollinaria & (Guenée, [1858]) & Maramalai & Feb 2012 \\
\hline 11 & Scopula & divisaria & Walker, 1861 & Upper Kothayar & Jun 2013 \\
\hline 13 & Scopula & fibulata & (Guenée, [1858]) & Kannikatti & Feb 2013 \\
\hline 14 & Scopula & $\mathrm{nr}$ relictata & (Walker, 1866) & Kuthiraivetti & Mar 2014 \\
\hline 15 & Scopula & $\mathrm{nr}$ actuaria & (Walker, 1861) & Sengeltheri & Feb 2015 \\
\hline 16 & Scopula & sp.6 & Schrank, 1802 & Upper Kothayar & Mar 2014 \\
\hline 17 & Somatina & nr plynusaria & (Walker, [1863]) & Sengeltheri & Oct 2012 \\
\hline 18 & Somatina & rosacea & Swinhoe, 1894 & Kuthiraivetti & Mar 2014 \\
\hline \multirow[t]{2}{*}{19} & Somatina or $\mathrm{nr}$ & & Guenée, [1858] & Kannikatti, Upper Kothayar & Feb 2013, Mar 2014 \\
\hline & & Sterrhini & Meyrick, 1892 & & \\
\hline 20 & Lophophleps & phoenicoptera & (Hampson, 1896) & Kalikesam & Jul 2014 \\
\hline 21 & Lophophleps & purpurea & Hampson, 1891 & Sengeltheri & Feb 2015 \\
\hline 22 & Idaea & gemmaria & Hampson, 1896 & Maramalai & Feb 2012 \\
\hline 23 & Idaea & $\mathrm{nr}$ gemmaria & Hampson, 1896 & Upper Kothayar & Mar 2014 \\
\hline 24 & Idaea & violaceae & Hampson, 1891 & Maramalai & Feb 2012 \\
\hline 25 & Idaea & sp.4 & Treitschke, 1825 & $\begin{array}{l}\text { Sengeltheri, Upper } \\
\text { Kothayar }\end{array}$ & $\begin{array}{l}\text { Oct 2012, Feb 2015, } \\
\text { Mar } 2014\end{array}$ \\
\hline 26 & Idaea & sp.5 & Treitschke, 1825 & Maramalai & Feb 2012 \\
\hline 27 & Idaea & sp.6 & Treitschke, 1825 & Maramalai & Feb 2012 \\
\hline 28 & Idaea & sp.7 & Treitschke, 1825 & Sengeltheri & Feb 2015 \\
\hline \multirow[t]{2}{*}{28} & Idaea & sp. 8 & Treitschke, 1825 & Kuthiraivetti & Mar 2014 \\
\hline & & Timandrini & Stephens, 1850 & & \\
\hline 29 & Timandra & sp. & Duponchel, 1829 & Upper Kothayar & Jun 2013 \\
\hline \multirow[t]{2}{*}{30} & Unidentified Sterrhinae -3 & & Meyrick, 1892 & Sengeltheri, Maramalai & Feb 2015, Feb 2012 \\
\hline & $\begin{array}{l}\text { Larentiinae } \\
\text { Duponchel, } 1845\end{array}$ & Asthenini & & & \\
\hline 31 & Acolutha & pictaria & (Moore, 1888) & Upper Kothayar & Mar 2014 \\
\hline 32 & Polynesia & sunandava & (Walker, 1861) & Kuthiraivetti & Dec 2012, Jan 2019 \\
\hline
\end{tabular}




\begin{tabular}{|c|c|c|c|c|c|}
\hline & S. Family/ Genus & Tribe/ Species & $\begin{array}{l}\text { Author \& Year: S. Family/ } \\
\text { Tribe/ Genus/ Species }\end{array}$ & Location & Month and Year of survey \\
\hline & & Cidariini & Duponchel, 1845 & & \\
\hline 33 & Ecliptopera & dissecta & (Moore, [1887]) & $\begin{array}{l}\text { Upper Kothayar, } \\
\text { Kuthiraivetti, }\end{array}$ & Jun 2013, Jan 2019 \\
\hline 34 & Ecliptopera & muscicolor & (Moore, 1888) & Upper Kothayar & Jun 2018 \\
\hline \multirow[t]{2}{*}{35} & Chloroclystis & sp. & Hübner, [1825] & Sengeltheri & Feb 2015 \\
\hline & & Incertae sedis & & & \\
\hline \multirow[t]{2}{*}{36} & Physetobasis & annulata & (Hampson, 1891) & Kuthiraivetti & Dec 2012 \\
\hline & & Eupitheciini & Tutt, 1896 & & \\
\hline 37 & Bosara & albitornalis & (Prout, 1958) & Kuthiraivetti & Dec 2012 \\
\hline 38 & Eupithecia & sp. & Curtis, 1825 & Sengeltheri & Feb 2015 \\
\hline 39 & Collix & sp.1 & Guenée, [1858] & Sengeltheri & Feb 2015 \\
\hline 40 & Collix & sp.2 & Guenée, [1858] & Marmalai & Feb 2012 \\
\hline 41 & Collix & sp.3 & Guenée, [1858] & Marmalai & Feb 2012 \\
\hline 42 & Eois & sp.4 & Hübner,1818 & Marmalai & Feb 2012, 2015 \\
\hline 43 & Eois & sp. 5 & Hübner,1818 & Upper Kothayar & Mar 2014 \\
\hline 44 & Eois & cf. dissimilis & (Moore,1887) & Kuthiraivetti & Dec 2012 \\
\hline 45 & Eois & $\begin{array}{l}\text { lunulosa } \\
\text { form ochraceae }\end{array}$ & (Moore, [1887]) & Kuthiraivetti & Dec 2012 \\
\hline 46 & Gymnoscelis & cf. admixtaria & (Walker, 1862) & Kuthiraivetti & Dec 2012 \\
\hline \multirow[t]{2}{*}{47} & Ziridava & rubridisca & (Hampson, 1891) & Upper Kothayar & Oct 2018 \\
\hline & & Trichopterygini & Warren, 1894 & & \\
\hline 48 & Sauris & sp.1 & Guenée, [1858] & Kuthiraivetti & Dec 2012 \\
\hline \multirow[t]{2}{*}{49} & Sauris & sp. 2 & Guenée, [1858] & Sengeltheri & Oct 2012 \\
\hline & & Xanthorhoini & Pierce, 1914 & & \\
\hline 50 & Xanthorhoe & saturata & Guenée, [1858] & Kuthiraivetti & Dec 2012 \\
\hline \multirow[t]{2}{*}{51} & Unidentified Eupethiciini-3 & & Duponchel, 1845 & Kuthiraivetti & Dec 2012, Mar 2014 \\
\hline & $\begin{array}{l}\text { Geometrinae } \\
\text { Stephens,1829 }\end{array}$ & Agathiini & Ban \& Han, 2018 & & \\
\hline 52 & Agathia & hemithearia & Guenée, [1858] & $\begin{array}{l}\text { Kuthiraivetti, Upper } \\
\text { kothayar }\end{array}$ & Mar 2014, Jan 2019 \\
\hline 53 & Agathia & lycaenaria & (Kollar, 1844) & Upper Kothayar & Jul 2018 \\
\hline \multirow[t]{2}{*}{54} & Agathia & laetata & (Fabricius, 1794) & Kuthiraivetti & Dec 2012 \\
\hline & & Archaeobalbini & Viidalepp, 1981 & & \\
\hline 55 & Herochroma & cf. cristata & Warren, 1894 & $\begin{array}{l}\text { Maramalai, Upper } \\
\text { Kothayar }\end{array}$ & Feb 2012, Mar 2014 \\
\hline \multirow[t]{2}{*}{56} & Lophophelma & ruficosta & Hampson, 1891 & Kuthiraivetti, Sengeltheri & $\begin{array}{l}\text { Dec 2012, Mar 2014, } \\
\text { Jan 2019, Feb } 2015\end{array}$ \\
\hline & & Comibaenini & Inoue, 1961 & & \\
\hline 57 & Argyrocosma & inductaria & (Guenée, [1858]) & $\begin{array}{l}\text { Kuthiraivetti, Upper } \\
\text { Kothayar, Sengeltheri, } \\
\text { Talayanai, Maramalai }\end{array}$ & $\begin{array}{l}\text { Dec 2012, Feb 2015, } \\
\text { Feb } 2012\end{array}$ \\
\hline 58 & Chlorochromodes & sp. & Warren, 1896 & Talayanai & Feb 2015 \\
\hline 59 & Comibaena & attenuata & (Warren, 1896) & Upper Kothayar & Mar 2014 \\
\hline 60 & Comibaena & cassidara & Guenée, [1858]) & $\begin{array}{l}\text { Mundanthurai, Upper } \\
\text { Kothayar, Maramalai }\end{array}$ & $\begin{array}{l}\text { Mar 2012, Oct 2016, } \\
\text { Feb } 2012\end{array}$ \\
\hline 61 & Comibaena & cf. striataria & Leech, 1897 & Mundanthurai & Mar 2012 \\
\hline 62 & Comibaena & integranota & Hampson, 1893 & Sengeltheri, Maramalai & Feb 2015, Feb 2012 \\
\hline 63 & Comibaena & fuscidorsata & Prout, 1912 & $\begin{array}{l}\text { Upper Kothayar, } \\
\text { Kuthiraivetti }\end{array}$ & Mar 2016, Jan 2019 \\
\hline 64 & Protuliocnemis & cf. biplagiata & (Moore, [1887]) & $\begin{array}{l}\text { Kuthiraivetti, Upper } \\
\text { Kothayar, Sengeltheri, } \\
\text { Maramalai }\end{array}$ & $\begin{array}{l}\text { Dec 2012, Mar 2014, } \\
\text { Oct 2012, Feb } 2012\end{array}$ \\
\hline
\end{tabular}




\begin{tabular}{|c|c|c|c|c|c|}
\hline & S. Family/ Genus & Tribe/ Species & $\begin{array}{l}\text { Author \& Year: S. Family/ } \\
\text { Tribe/ Genus/ Species }\end{array}$ & Location & Month and Year of survey \\
\hline \multirow[t]{2}{*}{65} & Protuliocnemis & partita & (Walker, 1861) & Upper Kothayar & Jan 2019 \\
\hline & & Dysphaniini & Warren, 1895 & & \\
\hline \multirow[t]{2}{*}{66} & Dysphania & percota & (Swinhoe, 1891) & Kalikesam & Jul 2014 \\
\hline & & Geometrini & Stephens, 1829 & & \\
\hline \multirow[t]{2}{*}{67} & Cyclothea & disjuncta & (Walker, 1861) & Sengeltheri & Feb 2015 \\
\hline & & Hemitheini & Bruand, 1846 & & \\
\hline 68 & Comostola sp & sp. & Meyrick, 1888 & $\begin{array}{l}\text { Sengeltheri, Kuthirai-vetti, } \\
\text { Maramalai }\end{array}$ & $\begin{array}{l}\text { Oct 2012, Jan 2019, } \\
\text { Feb } 2012\end{array}$ \\
\hline 69 & Episothalma & robustaria & (Guenée, [1858]) & Upper Kothayar & Dec 2011 \\
\hline 70 & Hemithea & tritonaria & (Walker, [1863]) & Upper Kothayar & Jul 2018 \\
\hline 71 & Hemithea & wuka & (Pagenstecher, 1886) & Kalikesam & Jul 2014 \\
\hline 72 & Idiochlora & nr caudularia & (Guenée, [1858]) & Kuthiraivetti, Sengeltheri & Mar 2014, Feb 2015 \\
\hline 73 & Orothalassodes & hypocrites & (Prout, 1912) & $\begin{array}{l}\text { Kuthiraivetti, Upper } \\
\text { Kothayar }\end{array}$ & Jan 2019 \\
\hline 74 & Pelagodes & sp. & Holloway, 1996 & All sites except Talayanai & $\begin{array}{l}\text { Mar 2011, Dec 2012, } \\
\text { Mar 2013, June 2014, } \\
\text { Feb 2012,'15, Jan } 2019\end{array}$ \\
\hline 75 & Pentheochlora & cf. uniformis & Hampson, 1891 & Kuthiraivetti & Dec 2012 \\
\hline 76 & Spaniocentra & sp. & Prout, 1912 & Maramalai & Mar 2012 \\
\hline 77 & Berta & cf. chrysolineata & Walker, 1863 & Upper Kothayar & Oct 2018 \\
\hline 78 & Jodis & nr undularia & (Hampson, 1891) & Upper Kothayar & Mar 2014 \\
\hline 79 & Jodis & pallescens & (Hampson, 1891) & Upper Kothayar & Mar 2014 \\
\hline 80 & Jodis & sp. 3 & Hübner, [1823] & Kuthiraivetti & Jun 2013 \\
\hline 81 & Microloxia & indecretata & (Walker, [1863]) & Talayanai & Feb 2015 \\
\hline \multirow[t]{2}{*}{82} & Microloxia & sp.2 & Warren, 1893 & Mundanthurai & Mar 2012 \\
\hline & & Nemoriini & Gumppenberg, 1887 & & \\
\hline 83 & Eucyclodes & gavissima & (Walker, 1861) & Sengeltheri & Oct 2012 \\
\hline \multirow[t]{2}{*}{84} & Eucyclodes & albisparsa complex & (Walker, 1861) & Sengeltheri & Oct 2012 \\
\hline & & Ornithospilini & Ban \& Han, 2018 & & \\
\hline 85 & Ornithospila & lineata & (Moore, 1872) & Kuthiraivetti & Jan 2019 \\
\hline \multirow[t]{2}{*}{86} & Ornithospila & submonstrans & (Walker, 1861) & Kuthiraivetti & Dec 2012, Jan 2019 \\
\hline & & Pseudoterpnini & Warren, 1893 & & \\
\hline 88 & Pingasa & dispensata $\mathrm{M}, \mathrm{F}$ & (Walker, 1866) & Kuthiraivetti & Dec 2012 \\
\hline 89 & Pingasa & ruginaria & (Guenée, [1858]) & $\begin{array}{l}\text { Upper Kothayar, } \\
\text { Sengeltheri, Kuthiraivetti, } \\
\text { Maramalai }\end{array}$ & $\begin{array}{l}\text { Mar 2014, Feb 2015, } \\
\text { Dec 2012, Jan 2019, } \\
\text { Feb 2012, Feb } 2015\end{array}$ \\
\hline \multirow[t]{2}{*}{90} & $\begin{array}{l}\text { Unidentified } \\
\text { Geometrinae-3 }\end{array}$ & & Leach, 1815 & Maramalai, Mundanthurai & Feb 2012, Mar 2012 \\
\hline & $\begin{array}{l}\text { Desmobathrinae } \\
\text { Meyrick,1886 }\end{array}$ & Desmobathrini & Meyrick, 1886 & & \\
\hline 91 & Noreia & ajaia $\mathrm{M}$ & (Walker, 1859) & Upper Kothayar & Jun 2013 \\
\hline 92 & Ozola & microniaria & Walker, 1862 & Kuthiraivetti, Sengeltheri & Mar 2014, Feb 2015 \\
\hline 93 & Ozola & sp. & Walker, 1861 & Sengeltheri & Feb 2015 \\
\hline \multirow[t]{2}{*}{94} & Derambila & fragilis & (Butler, 1881) & Sengeltheri & Feb 2015 \\
\hline & $\begin{array}{l}\text { Ennominae } \\
\text { Duponchel,1845 }\end{array}$ & Abraxini & Warren, 1894 & & \\
\hline 95 & Abraxas & leucostola argyrosticta & Hampson, 1893 & $\begin{array}{l}\text { Upper Kothayar, } \\
\text { Kuthiraivetti, Maramalai }\end{array}$ & $\begin{array}{l}\text { Jan 2019, Dec 2012, } \\
\text { Feb } 2012\end{array}$ \\
\hline 96 & Abraxas & fasciaria & Guerin-Meneville, 1843 & $\begin{array}{l}\text { Sengeltheri, Upper } \\
\text { Kothayar, Kuthiraivetti, }\end{array}$ & Oct. 2012, Jan 2019 \\
\hline 97 & Abraxas & Poliostrota $\mathrm{M}, \mathrm{F}$ & Hampson, 1907 & Kuthiraivetti & Dec. 2012 \\
\hline
\end{tabular}




\begin{tabular}{|c|c|c|c|c|c|}
\hline & S. Family/ Genus & Tribe/ Species & $\begin{array}{l}\text { Author \& Year: S. Family/ } \\
\text { Tribe/ Genus/ Species }\end{array}$ & Location & Month and Year of survey \\
\hline 98 & Abraxas & (grey)sp.4 & Leach, [1815] 1830 & $\begin{array}{l}\text { Upper Kothayar, } \\
\text { Kuthiraivetti, Sengeltheri }\end{array}$ & $\begin{array}{l}\text { Dec 2012, Oct 2013, } \\
\text { Feb 2015, Jan } 2019\end{array}$ \\
\hline 99 & Abraxas & (grey)sp.5 & Leach, [1815]1830 & Upper Kothayar & Mar 2014 \\
\hline 100 & Abraxas & (grey)sp.6 & Leach, [1815]1830 & Upper Kothayar, & Jan 2019, Feb 2012. \\
\hline 101 & Abraxas & (grey)sp.7 & Leach, [1815]1830 & Upper Kothayar & Jan 2019 \\
\hline 102 & Abraxas & (grey)sp. 3 forms & Leach, [1815]1830 & $\begin{array}{l}\text { Upper Kothayar, } \\
\text { Kuthiraivetti, Sengeltheri, } \\
\text { Maramalai. }\end{array}$ & $\begin{array}{l}\text { Jan 2019, June 2013, Mar } \\
\text { 2014, Feb 2015, Feb } 2012\end{array}$ \\
\hline \multirow[t]{2}{*}{103} & Abraxas & (yellow)sp.8 & Leach, [1815]1830 & $\begin{array}{l}\text { Upper Kothayar, } \\
\text { Sengeltheri }\end{array}$ & $\begin{array}{l}\text { Mar 2014, Mar 2019, } \\
\text { Feb } 2015\end{array}$ \\
\hline & & Baptini & Forbes, 1948 & & \\
\hline 106 & Borbacha & cf. pardaria & Guenée, [1858] & Kuthiraivetti, Maramalai & Dec 2012, Feb 2012 \\
\hline 107 & Synegia & imitaria & (Walker, 1861) & Upper Kothayar & Mar 2014 \\
\hline 108 & Yashmakia & erythra $\mathrm{M}$ & (Hampson, 1891) & Upper Kothayar & June 2018 \\
\hline 109 & Yashmakia & conflagrata $\mathrm{F}$ & (Hampson, 1912) & Upper Kothayar & June 2018 \\
\hline 110 & Lomographa & inamata & (Walker, [1861]1860) & Kuthiraivetti, Maramalai & $\begin{array}{l}\text { Dec 2012, Jan 2019, } \\
\text { Feb 2012 }\end{array}$ \\
\hline \multirow[t]{2}{*}{111} & Platycerota & vitticostata & (Walker, [1863]) & Upper Kothayar & June 2013 \\
\hline & & Boarmiini & Duponchel, 1845 & & \\
\hline 112 & Alcis & nilgirica & Hampson, 1891 & $\begin{array}{l}\text { Kuthiraivetti, Upper } \\
\text { Kothayar, Sengeltheri }\end{array}$ & $\begin{array}{l}\text { Dec 2012, June } 2013 \\
\text { Mar 2014, Oct 2012, } \\
\text { Feb } 2015\end{array}$ \\
\hline 113 & Amblychia & cf. angeronaria & Guenée, 1858 & $\begin{array}{l}\text { Maramalai, Upper } \\
\text { Kothayar }\end{array}$ & Feb 2012, June 2018 \\
\hline 114 & Amraica & recursaria & Walker, 1860 & Sengeltheri, & Feb 2015 \\
\hline 115 & Catoria & cf. sublavaria $\mathrm{F}$ & Guenée, [1858] & $\begin{array}{l}\text { Kuthiraivetti, Upper } \\
\text { Kothayar, Sengeltheri, }\end{array}$ & $\begin{array}{l}\text { Dec 2012, Feb 2015, } \\
\text { Jan } 2019\end{array}$ \\
\hline 116 & Chorodna & strixaria & (Guenée, [1858]) & Upper Kothayar & Mar 2014 \\
\hline 117 & Cleora & $\begin{array}{l}\text { alienaria } \mathrm{M} \\
5 \text { forms }\end{array}$ & Walker, 1860 & $\begin{array}{l}\text { Kuthiraivetti, Upper } \\
\text { Kothayar, Sengeltheri, } \\
\text { Marmalai }\end{array}$ & $\begin{array}{l}\text { Dec 2012, June 2013, } \\
\text { Jan 2019, Feb 2012, } 2015\end{array}$ \\
\hline 118 & Cleora & alienaria $\mathrm{F}$ & (Walker, 1860) & Kuthiraivetti & Jan 2019 \\
\hline 119 & Cleora & sp.3 & Curtis, 1825 & Kuthiraivetti & Jan 2019 \\
\hline 120 & Cleora & injectaria or $\mathrm{nr}$. & (Walker,1860) & Kuthiraivetti & Dec 2012 \\
\hline 121 & Ascotis & cf. imparata $\mathrm{F}$ & (Walker, 1860) & Kuthiraivetti & Dec 2012, Jan 2019 \\
\hline 122 & Cusiala & boarmoides & Moore, [1887] & Talayanai, Sengeltheri & Oct 2012, Feb 2015 \\
\hline 123 & Cusiala & raptaria & Walker, 1860 & Sengeltheri & Feb 2015 \\
\hline 125 & Cusiala & raptaria form disterminata & Walker, 1860 & Mundanthurai & Mar 2012 \\
\hline 126 & Cusiala & $\begin{array}{l}\text { raptaria distermi-nata- } \\
\text { variant }\end{array}$ & Walker, 1860 & Kuthiraivetti, Talayanai & Dec 2012, Feb 2015 \\
\hline 127 & Cusiala & $\begin{array}{l}\text { raptaria distermi-nata- } \\
\text { variant }\end{array}$ & Walker, 1860 & Kuthiraivetti, & Dec 2012 \\
\hline 128 & Cusiala & raptaria form rufifasciata & Walker, 1860 & Sengeltheri & Feb 2015 \\
\hline 129 & Cusiala & raptaria form suiasasa $\mathrm{M}$ & Walker, 1860 & $\begin{array}{l}\text { Mundanthurai, } \\
\text { Kuthiraivetti }\end{array}$ & Feb 2015, Dec 2012 \\
\hline 130 & Cusiala & raptaria-6 forms & Walker, 1860 & $\begin{array}{l}\text { Mundanthurai, } \\
\text { Kuthiraivetti, Talayani, } \\
\text { Sengeltheri }\end{array}$ & $\begin{array}{l}\text { Mar 2012, Oct 2012, Dec } \\
\text { 2012, Feb 2015, Jan } 2019\end{array}$ \\
\hline 131 & Ectropis & cf. dentilineata & (Moore, 1868) & Kuthiraivetti & Jan 2019 \\
\hline 132 & Ectropis & bhurmitra & (Walker, 1860) & Maramalai & Feb 2012 \\
\hline 133 & Dasyboarmia & cf. inouei & (Sato, 1987) & Sengeltheri, Kuthiraivetti & $\begin{array}{l}\text { Feb } 2015 \\
\text { March } 2014\end{array}$ \\
\hline 134 & Gasterocome & polyspathes & Prout,1934 & $\begin{array}{l}\text { Upper Kothayar, } \\
\text { Sengeltheri, Maramalai }\end{array}$ & $\begin{array}{l}\text { Mar 2014, Oct 2012, } \\
\text { Feb 2015, Feb } 2012\end{array}$ \\
\hline 135 & Hypomecis & pallida $1 \mathrm{M}, 4 \mathrm{~F}$ & (Hampson, 1891) & Upper Kothayar & Jun 2013 \\
\hline
\end{tabular}




\begin{tabular}{|c|c|c|c|c|c|}
\hline & S. Family/ Genus & Tribe/ Species & $\begin{array}{l}\text { Author \& Year: S. Family/ } \\
\text { Tribe/ Genus/ Species }\end{array}$ & Location & Month and Year of survey \\
\hline 136 & Hypomecis & trancissa & (Walker, 1860) & Maramalai & Feb 2012 \\
\hline 137 & Hypomecis & tamilensis & Sato, 2016 & Mundanthurai, Talayanai & Mar 2012, Feb 2015 \\
\hline 138 & Hypomecis & separata & (Walker, 1860) & Maramalai & Feb 2012 \\
\hline 139 & Hyposidra & talaca & (Walker, 1860) & $\begin{array}{l}\text { Upper Kothayar, } \\
\text { Kuthiraivetti, Maramalai }\end{array}$ & $\begin{array}{l}\text { Feb 2012, Dec } 2012 . \\
\text { Mar } 2014\end{array}$ \\
\hline 140 & Hyposidra & violescens $\mathrm{M}, \mathrm{F}$ & Hampson, 1895 & Kuthiraivetti, Maramalai & Dec 2012, Feb 2012 \\
\hline 141 & Hyposidra & sp. 3 & Guenée, [1858] & Upper Kothayar & Jan 2019 \\
\hline 142 & Ophthalmitis & cf. herbidaria & (Guenée, [1858]) & Sengeltheri & Oct 2012 \\
\hline 143 & Ophthalmitis & kalakadaria & Sato, 2014 & Sengeltheri & Feb 2015 \\
\hline 144 & Psilalcis & cf. subtochracea M, F & (Hampson, 1902) & $\begin{array}{l}\text { Kuthiraivetti, Sengeltheri, } \\
\text { Talayanai, Maramalai }\end{array}$ & $\begin{array}{l}\text { Dec 2012, Jan 2019, } \\
\text { Oct 2012. Feb 2015, } \\
\text { Feb } 2012\end{array}$ \\
\hline 145 & Psilalcis & $\mathrm{sp} .2$ & Warren, 1893 & Sengeltheri & Feb 2015 \\
\hline 146 & Psilalcis & sp. 3 & Warren, 1893 & Sengeltheri & Feb 2015 \\
\hline 147 & Psilalcis & sp.4 & Warren, 1893 & Maramalai & Feb 2012 \\
\hline 148 & Racotis & keralaria & Sato, 2004 & $\begin{array}{l}\text { Kuthiraivetti, Upper } \\
\text { Kothayar, Sengeltheri, } \\
\text { Maramalai }\end{array}$ & $\begin{array}{l}\text { Dec 2012, Jan 2019, } \\
\text { Feb 2015, Feb } 2012\end{array}$ \\
\hline 149 & Ruttellerona & cf. cessaria & (Walker, 1860) & Kuthiraivetti & Dec 2012 \\
\hline 150 & Ruttellerona & cf. pseudocessaria & Holloway, [1994] & Upper Kothayar & Jan 2019 \\
\hline \multirow[t]{3}{*}{151} & Biston & strigaria & (Moore, 1879) & $\begin{array}{l}\text { Kuthiraivetti, Sengeltheri, } \\
\text { Maramalai }\end{array}$ & $\begin{array}{l}\text { Dec 2012, Feb 2015, } \\
\text { Feb } 2012\end{array}$ \\
\hline & & Unidentified Boarmiini & Duponchel," 1845 & Kuthiraivetti & June 2013 \\
\hline & & Caberini & Duponchel, 1845 & & \\
\hline 152 & Astygisa & sp. & Walker, 1864 & Upper Kothayar & Mar 2014 \\
\hline 153 & Petelia & medardaria $\mathrm{M}$ & Herrich-Schäffer, [1856] & $\begin{array}{l}\text { Maramalai, Upper } \\
\text { Kothayar, Kuthiraivetti }\end{array}$ & $\begin{array}{l}\text { Feb 2012, Jun 2013, } \\
\text { Jan 2019, Dec 2012, } \\
\text { Mar 2014, Jan } 2019\end{array}$ \\
\hline 154 & Petelia & distracta $\mathrm{F}$ & (Walker, 1860) & $\begin{array}{l}\text { Kuthiraivetti, Sengeltheri, } \\
\text { Upper Kothayar }\end{array}$ & $\begin{array}{l}\text { Dec 2012, Oct 2012, } \\
\text { Feb 2015, Jan } 2019\end{array}$ \\
\hline 155 & Petelia & $\begin{array}{l}\text { immaculata } 2 \mathrm{M}, \\
2 \mathrm{~F}\end{array}$ & Hampson, 1893 & Kuthiraivetti & $\begin{array}{l}\text { Dec 2012, Mar 2014, } \\
\text { Jan } 2019\end{array}$ \\
\hline 156 & Petelia & fasciata & Moore, 1868 & & \\
\hline 157 & Petelia & sp. 3 & Herrich-Schäffer, 1855 & Kuthiraivetti & Dec 2012 \\
\hline \multirow[t]{2}{*}{158} & Hyperythra & lutea & (Stoll, [1781]) & Upper Kothayar & Mar 2014 \\
\hline & & Cassymini & Holloway, 1994 & & \\
\hline 159 & Heterostegane & subtessellata $\mathrm{M}, \mathrm{F}$ & (Walker, [1863]) & All sites & $\begin{array}{l}\text { Feb 2012, 2015, } \\
\text { Mar 2012, 2014, } \\
\text { June 2014, Jan } 2019\end{array}$ \\
\hline 160 & Heterostegane & cf. tritocampsis M, F & (Prout, 1934) & Sengeltheri, Talayanai & Feb 2015 \\
\hline 161 & Heterostegane & sp. 3 & Hampson, 1893 & Maramalai & Feb 2015 \\
\hline \multirow[t]{2}{*}{162} & Zamarada & cf. excisa & Hampson, 1891 & $\begin{array}{l}\text { Kuthiraivetti, Sengeltheri, } \\
\text { Maramalai }\end{array}$ & $\begin{array}{l}\text { Dec 2012, Oct 2012, } \\
\text { Feb } 2012\end{array}$ \\
\hline & & Ennomini & Duponchel, 1845 & & \\
\hline 163 & Ourapteryx & marginata & (Hampson, 1891) & $\begin{array}{l}\text { Kuthiraivetti, Kannikatti, } \\
\text { Upper Kothayar, } \\
\text { Sengeltheri, Maramalai }\end{array}$ & $\begin{array}{l}\text { Dec 2012, Feb 2013, } \\
\text { Mar 2014, Oct 2015, } \\
\text { Feb 2012, } 2015\end{array}$ \\
\hline \multirow[t]{2}{*}{164} & Ourapteryx & $\begin{array}{l}\text { peermaadiata-yellow and } \\
\text { white }\end{array}$ & Thierry-Mieg, 1903 & $\begin{array}{l}\text { Kuthiraivetti-yellow } \\
\text { Upper Kothayar-both }\end{array}$ & Mar 2014 \\
\hline & & Eutoeini & Holloway, 1994 & & \\
\hline 165 & Calletaera & postvittata & (Walker, 1861) & Upper Kothayar & Jun 2018 \\
\hline 166 & Luxiaria & emphatica & Prout, 1925 & Kuthiraivetti & Dec 2012 \\
\hline 167 & Luxiaria & hypaphanes M & Hampson, 1891 & $\begin{array}{l}\text { Sengeltheri, Kuthiraivetti, } \\
\text { Upper Kothayar }\end{array}$ & $\begin{array}{l}\text { Oct 2012, Dec 2012, } \\
\text { Jan 2019, Mar } 2014\end{array}$ \\
\hline
\end{tabular}




\begin{tabular}{|c|c|c|c|c|c|}
\hline & S. Family/ Genus & Tribe/ Species & $\begin{array}{l}\text { Author \& Year: S. Family/ } \\
\text { Tribe/ Genus/ Species }\end{array}$ & Location & Month and Year of survey \\
\hline 168 & Luxiaria & phyllosaria & (Walker, 1860) & Upper kothayar & Jan 2019 \\
\hline 169 & Luxiaria & sp.4 & Walker, 1860 & Kuthiraivetti & Jan 2019 \\
\hline 170 & Zeheba & $\mathrm{nr}$ aureata $2 \mathrm{M}, 1 \mathrm{~F}$ & Moore, [1887] & Sengeltheri, & Oct 2012, Feb 2015 \\
\hline \multirow[t]{2}{*}{171} & Zeheba & cf. aureatoides & Holloway, [1994] & Sengeltheri, Kuthiraivetti & Feb 2015, Dec 2012 \\
\hline & & Gonodontini & Forbes, 1948 & & \\
\hline \multirow[t]{2}{*}{172} & Gonodontis & pallida & (Butler, 1880) & Maramalai & Feb 2012 \\
\hline & & Hypochrosini & Guenée, 1858 & & \\
\hline 173 & Fasceliina & plagiata & (Walker, 1866) & $\begin{array}{l}\text { Upper Kothayar, } \\
\text { Maramalai, }\end{array}$ & Feb 2012 \\
\hline 174 & Fasceliina & $\begin{array}{l}\text { Chromataria-M5 forms, } \\
1 \mathrm{~F}\end{array}$ & Walker, 1860 & Upper Kothayar & Oct 2018 \\
\hline 175 & Achrosis & incitata complex-2 forms & (Walker, 1862) & $\begin{array}{l}\text { Kuthiraivetti, Upper } \\
\text { Kothayar }\end{array}$ & Mar 2014, Jan 2018 \\
\hline 176 & Achrosis & intexta & (Swinhoe, 1891) & Kuthiraivetti & Mar 2014 \\
\hline 177 & Achrosis & sp.4 4M & Guenée, [1858] & All sites & All survey years. \\
\hline 178 & Achrosis & $\mathrm{sp} .41 \mathrm{~F}$ & Guenée, [1858] & Kuthiraivetti, Sengeltheri & Dec 2012, Feb 2015 \\
\hline 179 & Achrosis & sp. $4 \mathrm{~F}$ & Guenée, [1858] & Kuthiraivetti & Dec 2012 \\
\hline 180 & Celenna & festivaria & (Fabricius, 1794) & Kannikatti, Upper Kothayar & Feb 2013, Mar 2014 \\
\hline 181 & Celenna & $\begin{array}{l}\text { festivaria and } \\
\text { form formosensis }\end{array}$ & (Fabricius, 1794) & $\begin{array}{l}\text { Kuthiraivetti } \\
\text { Upper Kothayar }\end{array}$ & $\begin{array}{l}\text { Mar 2014, } \\
\text { Feb 2013, Mar } 2014\end{array}$ \\
\hline 182 & Corymica & deducta & (Walker, 1866) & Upper Kothayar & Jun 2018 \\
\hline 183 & Corymica & sp. & Walker, 1860 & Kannikatti & Feb 2013 \\
\hline 184 & Hypochrosis & $\begin{array}{l}\text { hyadaria- chlorozonaria } \\
\text { complex-3 forms }\end{array}$ & $\begin{array}{l}\text { (Guenée, [1858]) - } \\
\text { (Walker, 1861) }\end{array}$ & $\begin{array}{l}\text { Kuthiraivetti, Upper } \\
\text { Kothayar, Sengeltheri, } \\
\text { Maramalai }\end{array}$ & $\begin{array}{l}\text { Dec 2012, Mar 2014, } \\
\text { Feb 2015, } \\
\text { Feb 2012, } 2015\end{array}$ \\
\hline 185 & Omiza & miliaria F-3 forms & Swinhoe, 1890 & $\begin{array}{l}\text { Kuthiraivetti, Upper } \\
\text { Kothayar, Sengeltheri, } \\
\text { Maramalai }\end{array}$ & $\begin{array}{l}\text { Dec 2012, Mar 2014, Oct } \\
\text { 2012, Feb 2015, } \\
\text { Feb 2012, Jan } 2019\end{array}$ \\
\hline \multirow[t]{2}{*}{186} & Omiza & miliaria $\mathrm{M}-2$ forms & Swinhoe, 1890 & Kuthiraivetti & Mar 2014 \\
\hline & & Incertae sedis & & & \\
\hline 187 & Eumelea & ludovicata M & Guenée, [1858] & Kuthiraivetti & Dec 2012 \\
\hline \multirow[t]{2}{*}{188} & Eumelea & sp.2 & $\begin{array}{l}\text { Duncan [\&Westwood], } \\
1841\end{array}$ & $\begin{array}{l}\text { Kuthiraivetti, } \\
\text { Sengeltheri }\end{array}$ & $\begin{array}{l}\text { Dec } 2012 \\
\text { Feb } 2015\end{array}$ \\
\hline & & Macariini & Guenée, 1858 & & \\
\hline 189 & Acanthovalva & sp. & Krüger, 2001 & Talayanai & Feb 2015 \\
\hline 190 & Chiasmia & eleonora & (Cramer, [1780]) & $\begin{array}{l}\text { Kuthiraivetti, Upper } \\
\text { Kothayar, Sengeltheri }\end{array}$ & $\begin{array}{l}\text { Dec 2012, Mar 2014, } \\
\text { Feb 2015, }\end{array}$ \\
\hline 191 & Chiasmia & inchoata & Walker, 1861 & Maramalai, Kuthiraivetti & $\begin{array}{l}\text { Feb 2012, Dec2012, } \\
\text { Feb2015 }\end{array}$ \\
\hline 192 & Chiasmia & myandaria & (Walker, [1863]) & Maramalai & Feb 2012 \\
\hline 193 & Chiasmia & nora & (Walker, [1861]) & $\begin{array}{l}\text { Kuthiraivetti, Upper } \\
\text { Kothayar, Sengeltheri }\end{array}$ & $\begin{array}{l}\text { Dec 2012, Mar 2014, } \\
\text { Feb } 2015\end{array}$ \\
\hline 194 & Chiasmia & ornatataria & (Leech, 1897) & Maramalai & Feb 2012 \\
\hline 195 & Chiasmia & ozararia & (Walker, [1860]) & Upper Kothayar & Oct 2018 \\
\hline 196 & Chiasmia & perfusaria & (Walker, 1866) & Kuthiraivetti & Dec 2012 \\
\hline 197 & Chiasmia & triangulata & (Hampson, 1891) & Maramalai & Feb 2012 \\
\hline 198 & Chiasmia & cf. normata. & (Walker, 1861) & Talayanai & Feb 2015 \\
\hline 199 & Chiasmia & sp.11 & Hübner, [1823] & Maramalai & Feb 2012 \\
\hline 200 & Chiasmia & sp.12 & Hübner, [1823] & Kuthiraivetti & Dec 2012 \\
\hline 201 & Chiasmia & sp.13 & Hübner, [1823] & Maramalai & Feb 2012 \\
\hline 202 & Chiasmia & sp.14 & Hübner, [1823] & Kuthiraivetti, Sengeltheri & Dec 2012, Feb 2015 \\
\hline 203 & Isturgia & disputaria group & Krüger, 2001 & Maramalai & Feb 2012 \\
\hline
\end{tabular}




\begin{tabular}{|l|l|l|l|l|l|}
\hline & S. Family/ Genus & Tribe/ Species & $\begin{array}{l}\text { Author \& Year: S. Family/ } \\
\text { Tribe/ Genus/ Species }\end{array}$ & Location & Month and Year of survey \\
\hline 204 & Isturgia & disputaria & Plutodini & Warren, 1894 & Talayanai \\
\hline 205 & Plutodes & nilgirica & Hampson, 1891 & $\begin{array}{l}\text { Kuthiraivetti, Upper } \\
\text { Kothayar }\end{array}$ & $\begin{array}{l}\text { Dec 2012, June 2013, } \\
\text { Jan 2019 }\end{array}$ \\
\hline 206 & Plutodes & pseudocyclaria & Kirty \& Goyal, 2011 & $\begin{array}{l}\text { Kuthiraivetti, Upper } \\
\text { Kothayar }\end{array}$ & Dec 2012, Jan 2019 \\
\hline 207 & Aplochlora & Scardamiini & Warren, 1894 & Karren, 1893 & Kothayar, Kuthiraivetti \\
\hline 208 & Scardamia & sp. 2 forms & metallaria & Guenée, [1858] & Kuthiraivetti \\
\hline
\end{tabular}

and A. latizonata Hampson, 1907, being white with grey or black pattern, are less similar. All the other Abraxas species from southern India show rather untypical coloration, grey or brown or yellow, sometimes with shining surface. Moths of the genus Abraxas are known to be toxic and their conspicuous appearance is a very significant and successful signal to predators which therefore avoid them, hence their abundance in many habitats, and presence of more than a hundred species across Europe to Australia. Why do species of southern Indian Abraxas not exhibit the typical kind of mimicry but prefer a rather mimetic appearance is a point for future research?

\section{Tribe Boarmiini Duponchel, 1845}

Plate 7-10 (130-190)

\section{Cleora Curtis, 1825}

Plate 8 (138-146)

Individuals identified as Cleora alienaria (Walker, 1860), (Plates 8 (138-144)) were quite common in KMTR. They were especially abundant in higher altitudes where they were recorded during most months of the year, except during April and May. The species has conspicuous pattern elements and their identity was determined with the help of literature sources and comparison with specimens in the ZFMK collection. Males were seen to be polymorphic; we have recorded five different variants which made identification even more difficult. A further problem is the existence of a very similar species, $C$. fraterna (Moore, 1888), described from Sikkim. Cleora alienaria was described from Sri Lanka and has a generally more southern distribution, but $C$. fraterna may perhaps occur also in the south. Externally both are not distinguished with certainty without details of their genitalia, which are very different. As there is no existing record of fraterna's presence in the south we report these as Cleora alienaria.
Cleora acaciaria (Boisduval, 1833), reported in Goyal's PhD thesis (Goyal 2010; PI. 36) from the Western Ghats is a misidentification of $C$. alienaria, as is evident from the comparison of genitalia figures depicted in the thesis and in Holloway, [1994] (Moths of Borneo, Part 11). The name $C$. acaciaria is valid today only for the species flying on Réunion Islands, extending perhaps to Mauritius.

Cleora $\mathrm{nr}$ injectaria (Walker 1860), (Plate 8 (145)), recorded druing the surveys, could not be confirmed to be true injectaria from the available information including distribution ranges, though it looks very similar to the typical, dark grey form of injectaria that is known to fly in montane habitats, from where this particular specimen was photographed. It is tentatively identified to the nearest possible species as $C$. $\mathrm{nr}$ injectaria for further investigation. True $C$. injectaria is known to be a lowland species, occurring mostly at seashore-areas with mangrove and along riverines (Holloway 1994; Kendrick 2015).

Externally, Cleora sp. 3. (Plate $8(146)$ ) is a member of the Carecomotis-group (Fletcher, 1953), but it bears resemblance to several species of this group. Of these, C. falculata (Fletcher, 1953), and C. onycha amplissima (Fletcher 1953), were reported from erstwhile Travancore. The holotype and paratype of C. falculata and the paratype of C. onycha amplissima are from this region. The study site from which Cleora sp. 3 was observed and photographed was earlier part of Travancore. A very similar species of Cleora, also of the Carecomotis-group, was recorded as C. propulsaria by Goyal from southern India in his thesis (Goyal 2010, Plate 37). On comparison of the genitalia Plates of $C$. falculata, C. onycha amplissima (Fletcher, 1953), and C. propulsaria (Holloway, [1994]) with that presented in his thesis, it is seen that the species reported by him is falculata and not propulsaria. Cleora sp. 3, therefore, could belong to any one of these three species. Without 
genitalic information, this remains identified as another species of Cleora.

\section{Ascotis Hübner, 1825}

Plate 8 (147)

Ascotis cf. imparata (Walker, 1860) (Plate 8 (147), female) was a species difficult to identify. Pattern and coloration are extremely similar to $C$. alienaria (Walker, 1860), but this was ruled out since this individual had narrower wings and was larger in wingspan (>45 mm). In size, this wingspan compared well with that reported for C. fraterna (Moore, 1888). But without any discerning characters (males of Ascotis with simple antennae would be easy to distinguish from males of Cleora with strongly pectinated antennae) and with scarce evidence of the presence of fraterna in southern India we at first, provisionally identified it as a species near alienaria. After further comparative studies the $2^{\text {nd }}$ author could identify it as a female of $A$. imparata, described from Nepal and "Hindostan", although not yet recorded from southern India. We believe that the presence of imparata cannot be excluded and it is unlikely, that the South Indian individuals may be members of any other closely related species.

Examination of genitalia is needed to identify this moth which was observed frequently in KMTR.

\section{Cusiala Moore, 1887}

Plates 8-9 (148-160)

Thirteen individuals of Cusiala Moore are reported. There are two species of Cusiala that fly in India, $C$. boarmoides Moore, [1887] and C. raptaria Walker, 1860. Both are polymorphic but at KMTR the latter was observed to be more widely polymorphic. Cusiala boarmoides, the type species described from Sri Lanka, flies both in northern and southern India but $C$. raptaria is reported only from southern India (Hampson 1895). The only distinguishable external difference between the two species (Moore 1887; Hampson 1895) is the postmedial band of the hindwing. In boarmoides, it is angled beyond the cell instead of curved as in raptaria. In the absence of any other clearly identifiable characteristics based on external morphology, to differentiate boarmoides from the typical raptaria of southern India, we have presented one individual as C. boarmoides (Plate $8(148)$ ), and all others are designated $C$. raptaria.

Hampson (1895) determined the species disterminata, rufifasciata and suiasasa as forms of c. raptaria. Our elementary investigation suggests that these determinations, too, need investigation. In addition, the sexual polymorphism, that this genus displays, too, needs investigation. These three forms were not the only ones that we found in our study sites. There were variants in the form disterminata, a black variant form, and several other with variable wing markings were observed. Twelve Cusiala rapataria that includes the described and undescribed forms (Plates 8-9 (149-160)) are reported. The existence of a wide variety of previously undescribed forms among C. raptaria require a more thorough investigation of the genitalic features to understand this genus and its species, as well as its sexual variations. Several other variants seen have been omitted from this paper, for want of detailed descriptions. This further underscores the importance of permitting collection to correctly document the diversity and furthering the scientific knowledge.

\section{Hypomecis Hübner, 1821}

Plate 9 (164-171)

Four species of Hypomecis - H. transcissa (Walker, 1860), H. separata (Walker, 1860), H. tamilensis Sato, 2016 and Hypomecis pallida (Hampson, 1891), are reported in this paper. Dark and light forms of $H$. separata are reported from the buffer area of the reserve. Hypomecis tamilensis is a species that was described from KMTR by Sato (2016). H. pallida was quite abundant in our study sites. The females of this species are polymorphic (Plate 9 (164-167)) and were found more abundantly and frequently than males. The postmedial fasciae on their wings varied from shades of light and dark grey with brownish or blackish tinge.

H. pallida had been earlier described as a member of the genus Narapa Moore, 1887, which later was placed as a junior synonym under Hypomecis (Scoble 1999; Hausmann, pers. comm. 29.vi.2019). Preliminary studies of the type-species of Narapa, N. adamata Felder \& Rogenhofer, 1875, from Sri Lanka, by the second author ( $2^{\text {nd }}$ author, unpublished data), which included analysis of male genitalia indicate that Narapa should be treated as a distinct genus in future again.

Several individuals that bore close resemblance to pallida, but differing in size, wing shape and markings were observed in the study sites, especially at Kuthiraivetti and Upper Kothayar. We believe that collection and further investigation is likely to reveal new data.

\section{Tribe: Caberini Duponchel, 1845}

Plate 10 (191-200)

Ten individuals, belonging to three genera, namely Astygisa Walker, 1864, Petelia Herrich-Schäffer, 1855, and Hyperythra Guenée, [1858], are reported. 


\section{Petelia Herrich-Schäffer, 1855}

Plate 10 (191-198)

Members of this genus were common in KMTR and were sighted during all surveys at all sites except Talayanai. Four species of Petelia - Petelia medardaria Herrich-Schäffer, [1856], P. immaculata Hampson, 1893, P. distracta (Walker,1860), and. P. fasciata Moore, 1868 , are reported in this paper. One individual could not be identified to species level. All four species are differentiated by the pattern of lines and spots on the wings.

Three almost straight, and almost parallel transverse lines in the forewing are typical to medardaria (Plate 10 (191)). Black apical patches are sometimes present in the forewings.

A curved medial line in the forewing, curved toward apex near costa and towards base near hind-margin is typical to immaculata. Females of Petelia species show wide variation, which the second author has noticed from the collections at ZFMK. This was evident in the two individuals of immaculata we have presented. The elements of black pattern near forewing apex which is typical of the females in immaculata (Hampson, 1893) was present in one and absent in another female (Plate 10 (196-197)). This pattern is lacking in males.

Identification of $P$. distracta (Plate 10 (192)), was difficult as the facies of distracta bears resemblance with that of medardaria female and $P$. delostigma Prout, 1932. The fasciae, however, have some discernable differences. The medial line in delostigma is curved more towards the tornus unlike in distracta in which it runs straight towards the inner margin. Petelia delostigma is larger than distracta and has not been reported from India. The black spots in distracta lie very close to the medial line almost touching it, whereas in the female of medardaria the spots lie well below the medial line, closer to the outer margin of the HW. Given these differences we conclude that the individual is $P$. distracta.

In P. fasciata (Plate 10 (193)), the antemedial and medial line of the forewing are diffused and rufousbrown with the postmedian band also being rufous brown, but ill-defined and waved. Diffused transverse greyish fascies between the rufous bands on both wings, a grey centred blackish discal spot in the HW and a marginal row of black spots form the markings of this species.

The markings on the Petelia sp. 5 (Plate 10 (198)) bear similarity to immaculata, but the presence of additional patterns on the hindwings require a more thorough investigation, hence it remains unidentified at the species level.

\section{Tribe: Ennomini Duponchel, 1845 \\ Ourapteryx Leach, 1814}

Plate 11 (207-209)

We report two species, the white Ourapteryx marginata (Hampson, 1891) (Plate 11 (207)), and the yellow Ourapteryx peermaadiata Thierry-Mieg, 1903 (Plate 11 (208)). The yellow O. peermaadiata was first described by Thierry-Mieg in 1903. Hampson (1907) described it again as Urapteryx ebuleata palniensis from Palani Hills, Tamil Nadu and Inoue (1993) redescribed peermadiata placing $O$. ebuleata palniensis as a junior synonym of peermaadiata. This is the only yellow coloured Ourpateryx that flies in southern India. The third author who is researching Ourapteryx species of India ( $3^{\text {rd }}$ author, unpublished data) has recorded a white morph of $O$. peermaadiata from southern India which has also been recorded in KMTR (Plate 11 (209)). Wingshape and all pattern elements are exactly the same in both forms, but genitalia have not been compared yet.

Comparisons of genitalia presented by Inoue (1993) with that reported by Goyal in his PhD thesis (Goyal 2010, Plate 57) show that O. peermaadiata has been incorrectly described as $O$ devikulamensis sp. $\mathrm{n}$.

\section{Tribe Eutoeini Holloway, 1994}

Plate 11 (210-219)

\section{Zeheba Moore, [1887]}

Plate 11 (216-219)

Four individuals that we report are only tentatively identified. The second author has compared these individuals (Plate $11(216-219))$ to the material of an undescribed Zeheba from Sri Lanka in the ZFMK collection. Based on external morphology, these individuals from KMTR resemble the material at the museum.

Males and females of Zeheba are easily distinguished. While both have simple, unpectinated antennae, the hindwing margin is dentate in males, but is smooth, broader and angled in the middle (at vein $\mathrm{M} 3$ ) in females (Plate 11 (216)). Zeheba marginata that Moore (1884) describes and figures in Lepidoptera of Ceylon (Moore, 1884-7) is a female of the yet-to-be described Zeheba species from Sri Lanka. True Z. marginata was described from Java by Walker in 1886 and is likely to be extralimital to India. The female we report is very similar to the female described by Moore, erroneously as Z. marginata.

The presence of this undescribed species in southern 
India is further confirmed from another erroneous record of Zeheba marginata in Goyal's PhD thesis (Goyal 2011; pl. 1). The genitalia details of the male presented in the thesis are identical to the male genitalia of the undescribed Sri Lankan material in ZFMK collection, which the second author has dissected and studied $\left(2^{\text {nd }}\right.$ author, unpublished data).

These individuals (Plate 11 (216-218)) also bear strong resemblance to $Z$. aureata Moore, [1887], a Himalayan species, but the genitalia of the aureata are quite distinctly different ( $2^{\text {nd }}$ author, unpublished data). We report them tentatively as Z. nr aureata but they require a detailed investigation to confirm the species identity.

The individual in Plate 11 (219) is a male which is larger than the other three Individuals, half-white (or pale-yellow), including the broader wing borders, and with almost-hyaline basal part of hindwing. Without genitalic details its identity cannot be determined with certainty, but likely belongs to Z. aureatoides Holloway, [1994], described from Borneo, but with a wider distribution to Sulawesi and Peninsular Malaysia (Holloway, [1994]; coll. ZFMK), Thailand, Myanmar (coll. ZFMK, unrecorded) and perhaps, as a new record, to southern India. Examination of specimens would be necessary to investigate further to prove this.

\section{Tribe Hypochrosini Guenée, 1858}

Plates 11-12 (221-246)

\section{Achrosis Guenée, [1858]}

Plates 11 (221-223); Plate 12 (226-230)

Several individuals of the genus Achrosis were observed, mostly in the wet evergreen or moist deciduous habitats. Of the seven individuals of this genus presented in this paper, $A$. intexta (Swinhoe, 1891) (Plate $11(223))$ is identified with certainty, because of its conspicuous pattern and coloration and as it is the only species of the intexta-group known from South India (type-locality: Kanara, southwestern India). Other species of the intexta-group are known from Peninsular Malaysia, Borneo and Sumatra (Holloway [1994], the Philippines (ZFMK, not yet recorded).

We report another two individuals (Plate 11 (221 \& 222)) with pattern and coloration of Achrosis incitata (Walker, 1862) (type-locality: northern India, Darjeeling). Both of them differ considerably from each other and may be members of two different species. From southern India (Nilgiri Hills), Swinhoe (1891) described Zomia miscella as new to science which Hampson (1895) synonymized with "Prionia" incitata, indicating that incitata flies throughout India. Swinhoe later opines that miscella is a southern form of incitata. A further species of this group was described from Sri Lanka as Timandra? serpentinaria (Walker, 1866), which certainly may also occur in the extreme south of India. Identification of two Individuals we recorded of the incitata-group is only possible by dissection of genitalia; hence further investigation is required.

Holloway (1994) described a new species close to $A$. incitata from Borneo and Sumatra and in the process reviewed what he calls the incitata complex. According to him almost all species of this complex are allopatric (except his new Bornean species which overlaps with the Sumatran species) and all are distinguished by differences in the male genitalia.

The two individuals we report as $A$. incitata (Plate 11 (221 \& 222)) may be one among the three species of incitata complex that fly in southern India or Sri Lanka or they could be a new species of the incitata complex. Further investigation is required.

We also report one unattributed species, Achrosis sp. 4 (Plate 12 (226-230)). The ZFMK, too, has specimens of this species that do not match any described so far in their collection. More than one form of the male was observed but only a single female was spotted during the survey. Neither Achrosis sp. 4 nor the incitata complex resemble Achrosis euchroes (Prout, 1917), described from Nilgiris, which also does not find mention in the incitata-complex described by Holloway [1994], as it did not occur in Borneo.

\section{Celenna festivaria (Fabricius, 1794)}

Plates 11-12 (224-225)

Two forms of Celenna festivaria Fabricius were found flying from March to August. They were found only in two sites of the survey (Table 3 ). The typical form of festivaria where the green patches are separated in the forewing, was less frequently seen than the form where the green bands were fused completely (Plate 11 (224)) and formed a large green patch on the FW. Although this species is common in India, there has been no report of racial differences so far. This form with the large fused bands that is dominant in Taiwan was named formosensis by Inoue (1964) and treated as a subspecies of festivaria. Holloway [1994] reports that the genitalia of ssp. formosensis described by Inoue resemble those of the Indian nominal subspecies $C$. festivaria festivaria and he therefore confirms it as a subspecies.

Celenna centraria Snellen, 1880, described from Sumatra, but also occurring in Borneo, is reported by Kirti et al. (2019) as found in the Andaman Islands. 
The fused pattern on the forewing of centraria is quite different from the species we are reporting here. Holloway [1994] states and figures that the genitalia of centraria differ strongly from those of festivaria. Until further investigation is undertaken, we report both forms as $C$. festivaria.

\section{Fascellina chromataria Walker, 1860}

Plate 12 (234-238)

These moths were seen from the month of June onwards till November. Four males and one female recorded is presented here. Sexual dimorphism is well marked; the female was yellowish-brown with cream coloured antemedial and postmedial lines. The postmedial line had cream spots at regular intervals. The wing colour in males ranged from shades of greenishbrown, brown to black (Plate 12 (235-238)). The submarginal fascia was white in three forms, while in the fourth, the brown form, there was merely a white speck near the excavated tornal margin (Plate 12 (237)). Each of the differently coloured forms were seen in different months and did not fly together.

The hindwing of the underside of the males were chrome-yellow, as their name suggests. The basal parts of the forewing were yellow, red-brown around the postmedial line; the postmedial regions being a mix of brownish-red. A greyish angled line and a patch near the apex of FW was also observed. Fascellina chromataria was described from Sri Lanka, and it has been reported from the Shendurney Wildlife Sanctuary in Kerala (Sondhi et al. 2018). Forests of KMTR and Shendurney Wildlife Sanctuary are part of Agasthyamalai Biosphere Reserve. The female seen in KMTR (Plate 12 (234)) is similar to the female collected and identified from Shendurney and therefore is identified as Fascellina chromataria. As we also observed more than one form of chromataria males, we report this group of four males and one female as Fascellina chromataria complex. Examination of specimens are needed to determine whether the individuals of this complex are morphs, subspecies or different species.

\footnotetext{
Hypochrosis hyadaria (Guenée, [1858]) chlorozonaria (Walker, 1861)

Plate 12 (239-241)

Hypochrosis hyadaria is treated at present as a single species with a large number of described subspecies (Holloway [1994]; Scoble et al. 1999), distributed allopatrically from India, over large areas of southeastern Asia and the Malayan archipelago. Hypochrosis chlorozonaria Walker, 1861, described from
}

Sri Lanka, is treated as a related, but distinct species. It was later described again as Numaria galbulata (Felder \& Rogenhofer, 1875) and Marcala sulphurescens (Moore, [1887]), from Sri Lanka. Hypochrosis galbulata and $H$. sulphurescens are just different forms of the same variable species, chlorozonaria. Unlike the description of hyadaria given by Hampson (1895), the facies described by Moore for these two forms of chlorozonaria match well with the facies of the individuals (Plate 12 (239241)) we have recorded. Holloway (1994) states, "India through S.E. Asia", as the geographical range for hydaria. Evidence of hyadaria flying in southern India comes from the surveys at Shendurney Wildlife Sanctuary by Yash Sondhi (Sondhi et al. 2018). It is possbile that both the species-hyadaria and chlorozonaria-could be found flying together in this region. Investigations through examination of the genitalia of collected specimens alone can provide their correct identity. Therefore, we report these three individuals as forms of Hypochrosis hyadaria or chlorozonaria.

\section{Tribe Macariini Guenée, 1858}

Plate 12-13 (250-269)

The identities of various moths in this tribe were investigated by referring to Krüger's (2001) extensive work on African species and a range of global macariine species, and the review of this tribe by Scoble \& Krüger (2002). The details given in the works of Hampson (1891, 1895), Moore (1884-7), and Walker (1862) were also consulted. Some macariine genera can be identified based on wing colour, shape, and pattern (Scoble \& Krüger 2002). However, genitalia structure is the most reliable feature for identification of moths from this tribe.

The macariine taxonomy is still in flux as several species from the Oriental region, including some from India, have not yet been described, as is evident from this paper. Nineteen macariine individuals have been observed and are classified here in three genera, namely, Acanthovalva Krüger, 2001, Chiasmia Hübner, [1823] 1816, and Isturgia Hübner, [1823] 1816.

\section{Acanthovalva Krüger, 2001 \\ Plate 12 (250) \\ The general features of this moth match the description given for Acanthovalva by Krüger (2001). Further investigation about the species requires collection and examination of genitalia. Although he could not examine any specimens, Krüger suggests that Tephrina fumosa (Hampson, 1895) from Nilgiris may be closely related to Acanthovalva bilineata (Warren, 1895)}


from South Africa, extending, if verified, the range of this genus to the Oriental region. This is the first record of the genus Acanthovalva from southern India.

\section{Chiasmia Hübner, [1823] 1816}

Plate 12-13 (251-266)

Fourteen macariine species belonging to the genus Chiasmia Hübner are reported. Four of them could be identified only to genus level. One is presented with a provisional identification and nine of them are identified to species level using the various published records listed throughout in this paper.

We report here that the ZFMK has a group of four specimens named C. ablataria Swinhoe, collected from the Nilgiri Hills. Externally, they bear close resemble to the unidentified Chiasmia sp. 13 (Plate 13 (265)). However, our research did not yield any more information, such as the year Swinhoe described this species, whether it is a synonym for a described taxon or any literature on it. The name is missing even from the list in Scoble et al. (1999.) This, again, underscores the importance of collecting moths for an accurate description and identification.

\section{Isturgia Hübner, [1823] 1816}

Plate 13 (267-269)

\section{Isturgia disputaria (Guenée, [1858])}

The various species of Isturgia have been broadly placed under five groups by Krüger (2001). One of them is the Isturgia disputaria group. We report two taxa that belong to it. Both are tentatively identified, as details of genitalia are not available.

We have identified a female from Talayanai (Plate 13 (269)) as Isturgia disputaria Guenée. It is identified by the strong, black fasciae of the forewings. The description of the fasciae matches that given by Hampson (1895). Hampson (1895) also states that the southern Western Ghats forms appear generally paler and ochreous. Krüger (2001) mentions that the HW median line in disputaria may be faint or absent, while Hampson records the HW "with sinuous median line". These lines are partly visible in our Plate, but minor variations in fascia could also be a geographical phenomenon, in our opinion.

The second taxon (Plate 13 (267-268)) we report as Isturgia-a male and a female observed at Maramalai (a higher elevation site in the buffer zone of the Reserve)also belongs to the I. disputaria group. It bears some resemblance to the former taxon, but has much weaker transverse fasciae. It may be just a form or variation of the latter, but may also be related to or conspecific with I. pulinda (Walker, 1860), from Sri Lanka. A year later, Walker (1861) described another Isturgia, I. deerraria, from South Africa, which was subsequently reported by several authors ((Agenjo 1974: 4; Herbulot 1978: 161; Fletcher 1978a: 77; Wiltshire 1952: 172; 1980: 197; 1990: 135; Hermosa 1985: 28; Hausmann 1991: 138) in Krüger (2001) as a subspecies of $I$. pulinda. Krüger (2001), after examining the genitalia of the typespecimens, concluded that the African species I. deeraria was not conspecific with I. pulinda. Based on the fasciae we believe that this Isturgia from Maramalai may be related to I. pulinda or may even be a new species. The real identity can only be decided after the study of the genitalia of collected Individuals.

Isturgia catalaunaria Guenée, [1858] was also listed as a macariine species from India (Kirti et al. 2019). Krüger (2001) has recorded this to be a misidentification, as this is a species from southern Spain and southern Africa, so extralimital to India.

\section{CONCLUSION}

Investigating 390 moths through photographs and without specimens in hand to refer to, was a daunting task. In the absence of specimens, we have attempted to assign as precise species identities as deemed possible, but many uncertainties in species identities can only be clarified through collection and examination of specimens. We have also attempted to compile the many historical records that lay scattered among different resources, and contemporary published papers on Geometridae, for easy access to future researchers. Many geometrids are flower feeders, hence important pollinators of plants in forests. Their presence is likely to help forests flourish. As most geometrids are polyphagous, their varied larval host plants are also crucial, and require protection of their habitats in the Western Ghats, an area of high endemism. Given the diversity observed by the first author in these forests, we hope that these preliminary findings will stimulate further research on the geometrid moths of KMTR.

\section{REFERENCES}

Anonymous 2021 - http://www.unesco.org/new/en/natural-sciences/ environment/ecological-sciences/biosphere-reserves/asia-and-thepacific/india/agasthyamala/ Last accessed 13 August 2021

Ban, X., N. Jiang, R. Cheng, D. Xue \& H. Han (2018). Tribal classification and phylogeny of Geometrinae (Lepidoptera: Geometridae) inferred from seven gene regions. Zoological Journal of the Linnean Society 184(3): 653-672. https://doi.org/10.1093/zoolinnean/zly013

Barlow, H.S. (1982). An introduction to the moths of S.E. Asia. Malayan Nature Society, Kuala Lumpur, 305 pp., 51 pls. 
Beccaloni, G., M. Scoble, I. Kitching, T. Simonsen, G. Robinson, B. Pitkin, A. Hine \& C. Lyal (Editors) (2003). The Global Lepidoptera Names Index (Leplndex). Last accessed 13 August 2021. https:// www.nhm.ac.uk/our-science/data/lepindex/lepindex/

Beljaev, E.A. (2007). Taxonomic changes in the emerald moths (Lepidoptera: Geometridae, Geometrinae) of East Asia, with notes on the systematics and phylogeny of Hemitheini. Zootaxa 1584: $55-68$.

Brechlin, R. (1998). Sechs neue indoaustralische Schwärmerarten (Lepidoptera, Sphingidae). Nachrichten des Entomologischen Vereins Apollo, Frankfurt am Main, N.F. 19(1): 23-42.

Brechlin, R. (2000). Zwei neue Arten der Gattung Loepa Moore, 1859 (Lepidoptera: Saturniidae). Nachrichten des Entomologischen Vereins Apollo, Frankfurt am Main, N.F. 21(3): 165-170.

Butler, A.G. (1881). Descriptions of new Genera and Species of Heterocerous Lepidoptera from Japan. The Transactions of the Entomological Society of London 1881: 1-25.

Butler, A.G. (1886). Illustrations of typical specimens of Lepidoptera Heterocera in the collection of the British Museum. Part VI, London, 89 pp., 19 pls.

Butler, A.G. (1889). Illustrations of typical specimens of Lepidoptera Heterocera in the collection of the British Museum. Part VII, London, 176 pp., 18 pls.

Choi, S.W. \& S.S. Kim (2013). Six new records of Idaea Treitschke (Lepidoptera: Geometridae, Sterrhinae) from Korea. Entomological Research 43: 27-33.

Choi, S.W. (2012). Taxonomic review of the genus Asthena Hübner (Lepidoptera: Geometridae) in Korea. Entomological Research 42 151-157. https://doi.org/10.1111/j.1748-5967.2012.00450.x

Cotes, E.C. \& C. Swinhoe (1887). A Catalogue of Moths of India. Pt. 1-Sphinges. The Trustees of the Indian Museum, Calcutta, $40 \mathrm{pp}$.

Cui, L., D. Xue \& N. Jiang (2019). A review of Organopoda Hampson, 1893 (Lepidoptera, Geometridae) from China, with description of three new species. Zootaxa 4651(3): 434-444. https://doi. org/10.11646/zootaxa.4651.3.2

Cui, L., D. Xue, N. Jiang (2019). A review of Timandra Duponchel, 1829 from China, with description of seven new species (Lepidoptera, Geometridae). ZooKeys 829: 43-74. https://doi.org/10.3897/ zookeys.829.29708

Dey, P., V.P. Uniyal, A. Hausmann \& D. Stüning (2021). Revision of the genus Prometopidia Hampson, 1902, with description of the new species $P$. joshimathensis sp. nov. from West-Himalaya and its subspecies $P$. $j$. yazakii ssp. nov. from Nepal (Lepidoptera: Geometridae, Ennominae). Zootaxa 4980(1): 28-44.

Elanchezhian, M., C. Gunasekaran \& A.A. Deepa (2014). A study on moth diversity in three different habitats of Maruthamalai Hill, Western Ghats, South India. Global Journal for Research Analysis 3(12): 136-138.

Fletcher, D.S. (1953). A revision of the genus Carecomotis (Lep. Geometridae), Annals and Magazine of Natural History: Series XII(6): 100-142. https://doi.org/10.1080/00222935308654403

Forum Herbulot (2003): The Forum Herbulot world list of family group names in Geometridae, <http://www.herbulot.de>, 11 pp., with updated version of 12.vi.2007 (A. Hausmann ed.). Last accessed on 13 August 2021

Govt. of Tamilnadu, Forest department (2009). Monitoring Primates - A guide for Kalakad Mundanthurai Tiger Reserve, https://www. forests.tn.gov.in/pages/view/kalakad_mundanthurai_tr. Last accessed on 13 August 2021.

Goyal, T. (2010). Taxonomic studies on family Geometridae (Lepidoptera) from Western Ghats of India, PhD thesis. Department of Zoology, Punjab Univeristy, 279 pp., 101 pls. Last accessed on 17 November 2020. http://hdl.handle.net/10603/2894

Goyal, T., J.S. Kirti \& A. Saxena (2018). Taxonomy of Genus Agathia Guenée (Lepidoptera: Geometridae), with description of a new species from Western Ghats, India. Indian Journal of Entomology 80(3): 951-959. https://doi.org/10.5958/0974-8172.2018.00144.X

Hampson, G.F. (1891). Illustrations of typical specimens of Lepidoptera Heterocera in the collection of the British Museum. Part VIII, Taylor
\& Francis, London, 144 pp., 18 pls.

Hampson, G.F. (1893). Illustrations of typical specimens of Lepidoptera Heterocera in the collection of the British Museum. Part IX, Taylor \& Francis, London, 182 pp., 21 pls.

Hampson, G.F. (1895). The Fauna of British India including Ceylon and Burma, Moths- Vol. 3. Taylor and Francis, London, 588 pp.

Hampson, G.F. (1896). The Fauna of British India including Ceylon and Burma, Moths- Vol. 4. Taylor and Francis, London, 632 pp.

Hampson, G.F. (1907). The Moths of India. Supplementary paper to the Volumes in "The Fauna of British India", Series III, Part IX. Journal of the Bombay Natural History Society 18: 27-53.

Hampson, G.F. (1912). The Moths of India. Supplementary paper to the Volumes in "The Fauna of British India". Series IV, Part IV. Journal of the Bombay Natural History Society 21(4): 1222-1272.

Han, H. \& D. Xue (2011). Thalassodes and related taxa of emerald moths in China (Geometridae, Geometrinae). Zootaxa 3019: 26-50.

Han, H., A.C. Galsworthy \& D. Xue (2009). A survey of the genus Geometra Linnaeus (Lepidoptera, Geometridae, Geometrinae). Journal of Natural History 43 (13-14): 885-922. https://doi. org/10.1080/00222930802702472

Han, H., A.C. Galsworthy \& D. Xue (2012). The Comibaenini of China (Geometridae: Geometrinae), with a review of the tribe. Zoological Journal of the Linnean Society 165: 723-772.

Holloway, J.D. (1993[4]). The moths of Borneo (Part 11); Family Geometridae: Subfamily Ennominae. Malayan Nature Journal 47: 1-309.

Holloway, J.D. (1996). The moths of Borneo (Part 9); Family Geometridae: Subfamilies Oenochrominae, Desmobathrinae, Geometrinae. Malayan Nature Journal 49: 147-326.

Holloway, J.D. (1997). The moths of Borneo (Part 10); Subfamilies Sterrhinae, Larentiinae. Malayan Nature Journal 51: 1-242.

Inoue, H. (1953). Notes on some Japanese Larentiinae and Geometrinae (Lepidoptera: Geometridae). Tinea 1: 1-18.

Inoue, H. (1964). Some new subspecies of the Geometridae from the Ryukyu Archipelago and Formosa (Lepidoptera). Kontyû 32(2): 335-340

Inoue, H. (1993). Redescription of Ourapteryx peermaadiata ThierryMieg from South India (Lepidoptera: Geometridae, Ennominae). Lepidoptera Science 44(3): 117-119. https://doi.org/10.18984/ lepid.44.3_117

Kendrick, R.C. (2002). Moths (Insecta: Lepidoptera) of Hong Kong. PhD Thesis. Department of Ecology and Biodiversity, Hong Kong, University of Hong Kong. 623 pp., 9 pls. http://hdl.handle. net/10722/31688. Last accessed on 4 December 2020

Kendrick, R.C. (2015). Polymorphism and populations: Cleora injectaria (Walker, 1860) (Lepidoptera: Geometridae) at Mai Po Nature Reserve, Hong Kong. Porcupine! 35: 8-10.

Kirti, J.S. \& T. Goyal (2011). A new species of Plutodes Guenée (Lepidoptera: Geometridae: Ennominae) from Western Ghats of India. Journal of Applied Biosciences 37(1): 68-70.

Krüger, M. (2001). A revision of the tribe Macariini (Lepidoptera: Geometridae: Ennominae) of Africa, Madagascar and Arabia. Bulletin of the Natural History Museum, London (Entomology Series) 70(1): 1-502.

Mathew, G. \& V.K. Rahamathulla (1995). Biodiversity in the Western Ghats - A study with reference to moths (Lepidoptera: Heterocera) in the Silent Valley National Park, India. Entomon 20(2): 25-33.

Moore, F. (1867). On the Lepidopterous Insects of Bengal, Part III. Proceedings of the Zoological Society of London 1867: 612-686.

Moore, F. (1879). Descriptions of New Indian Lepidopterous Insects from the collection of $W$. Atkinson. Heterocera, The Asiatic Society of Bengal. Calcutta, 350 pp., 8 pls.

Moore, F. (1884-7). The Lepidoptera of Ceylon. Volume 3. L. Reeve \& Co., London, 600 pp., 72 pls.

Murillo-Ramos, L., G. Brehm, P. Sihvonen, A. Hausmann, S. Holm, H.R. Ghanavi, E. Õunap, A. Truuverk, H. Staude, E. Friedrich, T. Tammaru \& N. Wahlberg (2019). A comprehensive molecular phylogeny of Geometridae (Lepidoptera) with a focus on enigmatic small subfamilies. PeerJ 7: e7386. https://doi.org/10.7717/ 
peerj.7386

Õunap, E., J. Viidalepp \& A. Truuverk (2016). Phylogeny of the subfamily Larentiinae (Lepidoptera: Geometridae): integrating molecular data and traditional classifications. Systematic Entomology 21(4): 824843. https://doi.org/10.1111/syen.12195

Plotkin, D. \& A.Y. Kawahara (2020). Review of recent taxonomic changes to the emerald moths (Lepidoptera: Geometridae: Geometrinae). Biodiversity Data Journal 8: e52190. https://doi. org/10.3897/BDJ.8.e52190

Prout, L.B. (1912). Lepidoptera, Heterocera. Family Geometridae, Subfamily Hemitheinae. Genera Insecta 129: 274 pp.

Prout, L.B. (1917). On new and insufficiently known Indo-Australian Geometridae. Novitates zoologicae 24: 293-317.

Prout, L.B. (1920-1941). The Indo Australian Geometridae. In: Seitz, A. (Ed.). The Macrolepidoptera of the World. Vol 12, Stuttgart Verlag des Seitz'schen Werkes (Alfred Kernen), 356 pp. 50 pls.

Prout, L.B. (1925). Geometrid descriptions and notes. Novitates Zoologicae 32: 31-69.

Prout, L.B. (1929). New species and sub-species of Geometridae. Novitates Zoologicae 35: 63-77.

Prout, L.B. (1929). On the Geometrid Genus Catoria Moore. Novitates Zoologicae 35: 132-141.

Prout, L. B. 1933. The Indoaustralian Geometridae, pp. 77-116. In: Seitz, A. (ed.) 1920-1934. The Macrolepidoptera of the World. Volume 12: 1-292.

Prout, L.B. (1934). Geometridae: Subfamilia Sterrhinae. Lepidopterorum Catalogus, part LXI: 1-180, W. Junk, Berlin.

Sato, R. (1987). Taxonomic notes on Menophra delineata (Walker) (Geometridae: Ennominae) and its allies from Indo-Malayan region. Tinea 12 Supplement: $249-258$.

Sato, R. (2004). Taxonomic notes on Racotis boarmiaria (Guenée) (Geometridae, Ennominae) and its allies from the Indo-Malayan region, with description of four new species. Tinea 18(2): 130-139.

Sato, R. (2014). Six new species of the genus Ophthalmitis (Geometridae, Ennominae) from Southeast Asia and South India, with taxonomic notes on the related species. Tinea 22(5): 318-330.

Sato, R. (2016). Five new species of the genera Hypomecis Hübner, Ophthalmitis Fletcher and Phthonosema Warren (Geometridae; Ennominae) from the Indo-Malayan region. Tinea 23(5): 241-248.

Schintlmeister, A. (2003). Die Gattung Stauropus Germar, 1812 (Lepidoptera: Notodontidae). Nachrichten des Entomologischen Vereins Apollo, Frankfurt/Main, N.F. 24(3): 97-118.

Scoble, J.M. \& M. Krüger (2004). A review of the genera of Macariini with a revised classification of the tribe (Geometridae: Ennominae). Zoological Journal of the Linnean Society 134: 257-315.

Scoble, M.J. (1999). Geometrid Moths of the World - A Catalogue (Lepidoptera: Geometridae), Vol. 1 \& 2, pp. 5-482 \& 485-1016 CSIRO Publishing, Collingwood.

Scoble, M.J. \& A. Hausmann [updated 2007]. Online list of valid and available names of the Geometridae of the World. Last accessed on 10 Jan 2021. http://www.herbulot.de/globalspecieslist.htm

Sihvonen, P. (2005). Phylogeny and classification of the Scopulini moths (Lepidoptera: Geometridae, Sterrhinae). Zoological Journal of the Linnean Society 143: 473-530.

Sihvonen, P., L.S. Murillo-Ramos, G. Brehm, H. Staude \& N. Wahlberg (2020). Molecular phylogeny of Sterrhinae moths (Lepidoptera: Geometridae): towards a global classification. Systematic Entomology 45: 606-634.

Sondhi, S., D. Nath, Y. Sondhi \& K. Kunte (2020). A new species of Metallolophia Warren, 1895 (Lepidoptera: Geometridae: Geometrinae) and notes on M. opalina (Warren, 1893) from eastern Himalaya, India. Zootaxa 4838(2): 289-297.

Sondhi, Y., S. Sondhi, S.R. Pathour \& K. Kunte (2018). Moth diversity (Lepidoptera: Heterocera) of Shendurney and Ponmudi in Agastyamalai Biosphere Reserve, Kerala, India, with notes on new records. Tropical Lepidoptera Research 28(2): 66-89. https://doi. org/10.5281/zenodo.2027709

Sondhi, S., Y. Sondhi, P. Roy \& K. Kunte (Chief Editors) (2021). Moths of India, v. 2.63. Indian Foundation for Butterflies.

Stüning, D. \& V.K. Walia (2009). The genus Astygisa Walker, 1864 in India, with description of a new species from western Himalaya (Lepidoptera: Geometridae, Ennominae). Tinea 21(1): 9-22.

Swinhoe, C. (1890). New species of moths from Southern India. Transactions of the Entomological Society of London 1891(1): 133154, pl.8. https://doi.org/10.1111/j.1365-2311.1891.tb01644.x

Thierry-Mieg, P. (1903). Descriptions de Lepidopteres Nocturnes. Annales de la Société entomologique de Belgique 47: 382-385.

Viidalepp, J. (2011). A morphological review of tribes in Larentiinae (Lepidoptera: Geometridae). Zootaxa 3136(1): 1-44. https://doi. org/10.11646/zootaxa.3136.1.1

Walker, F. (1860). List of the specimens of Lepidopterous insects in the collection of the British Museum, London. Part XX: 1-276.

Walker, F. (1860). List of the specimens of Lepidopterous insects in the collection of the British Museum, London, Part XXI: 1-373.

Walker, F. (1861). List of the specimens of Lepidopterous insects in the collection of the British Museum, London. Part XXII-XXV: 1-826.

Walker, F. (1862). List of the specimens of Lepidopterous insects in the collection of the British Museum, London. Part XXV-XXVI: 1-538.

Warren, W. (1894). New Genera and Species of Geometridae. Novitates Zoologicae, 1: 366-534.

Warren, W. (1896). New Species of Drepanidae, Thyrididae, Uraniidae, Epiplemidae, and Geometridae in the Tring Museum. Novitates Zoologicae 3: 335-420.

Warren, W. (1897). New Genera and Species of Geometridae. Novitates Zoologicae 4: 12-179 \& 195-306.

Xue, D., L. Cui \& N. Jiang (2018). A review of Problepsis Lederer, 1853 (Lepidoptera: Geometridae) from China, with description of two new species. Zootaxa 4392(1): 101-127. https://doi.org/10.11646/ zootaxa.4392.1.5

Xue, D., X. Wang \& H. Han (2009). A revision of Episothalma Swinhoe, 1893 , with descriptions of two new species and one new genus (Lepidoptera, Geometridae, Geometrinae). Zootaxa 2033: 12-25.

Yakovlev, R.V. (2009). New taxa of African and Asian Cossidae (Lepidoptera). Euroasian Entomological Journal 8(3): 353-361. 
Subfamily Sterrhinae: 5-36

Tribe Cosymbiini: 5-7

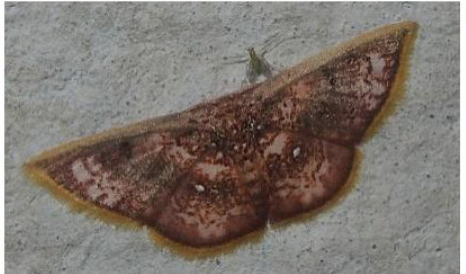

*5. Chrysocraspeda sp.1

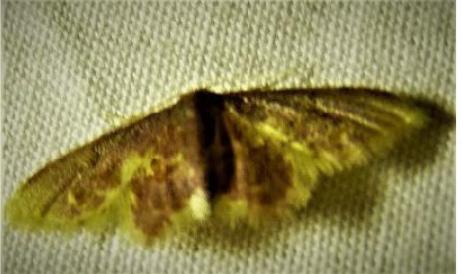

6. Chrysocraspeda sp.2

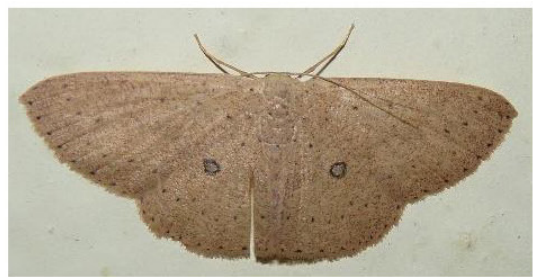

7. Perixera insitiva
Tribe Cyllopodini: 8

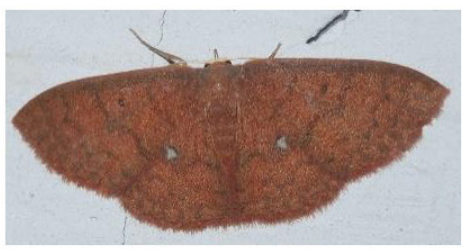

8. Organopoda sp.

Tribe Lissoblemmini:9-10

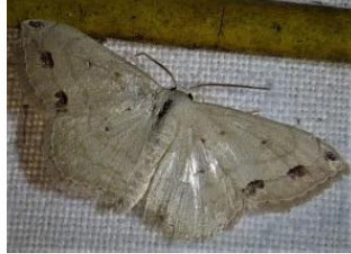

9. Craspediopsis sp.

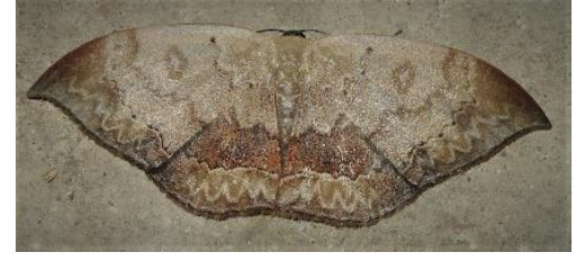

*10. Lissoblemma lunuliferata

Tribe Rhodometrini: 11-12
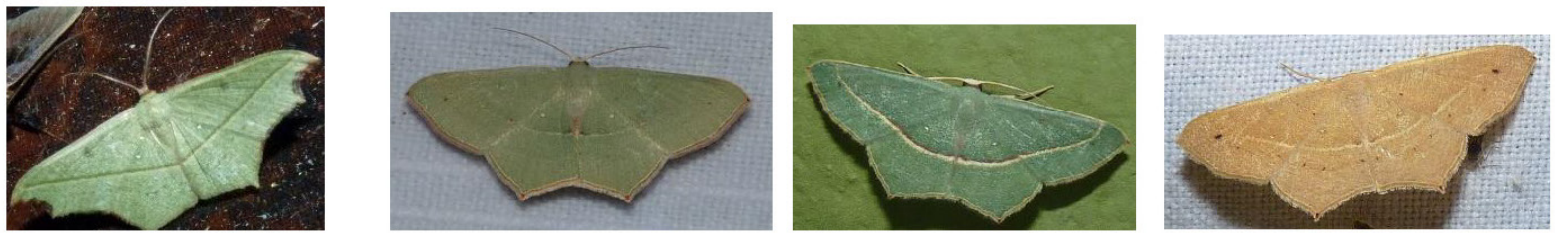

11. Traminda aventiaria 12

Traminda mundissima

Tribe Scopulini: 13-22

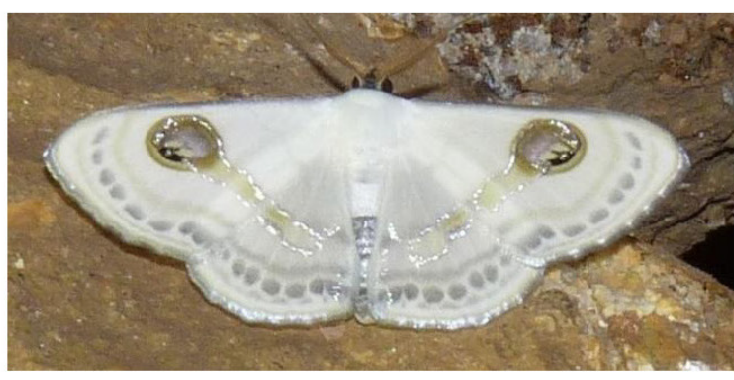

13. Problepsis apollinaria

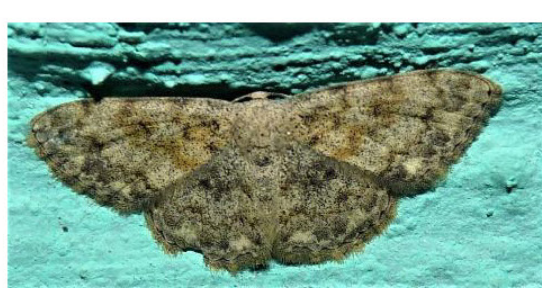

15. Scopula fibulata

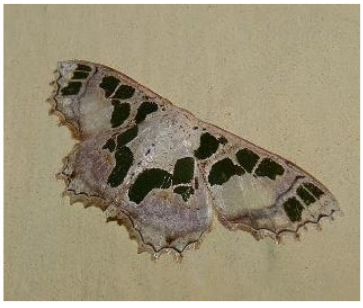

16. Scopula divisaria

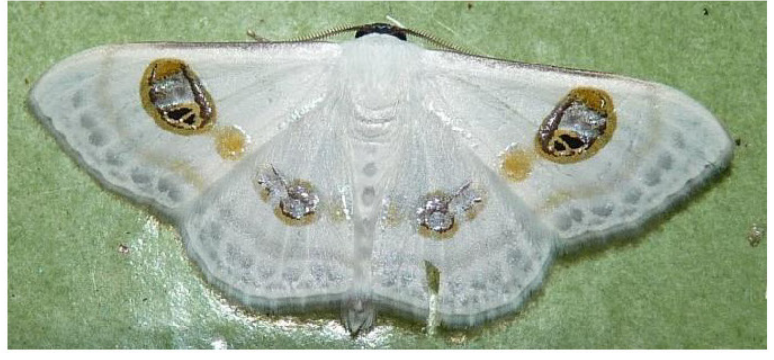

14. Problepsis deliaria

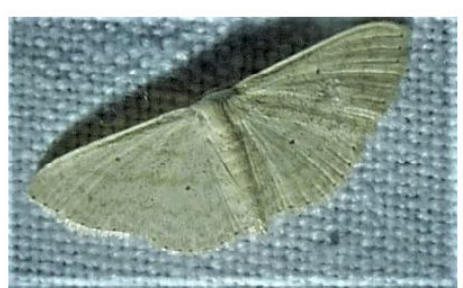

17. Scopula nr actuaria

Plate 1. Geometrids of Kalakad-Mundanthurai Tiger Reserve 


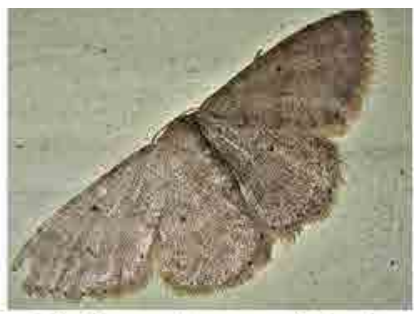

18. Scopula nr relictata

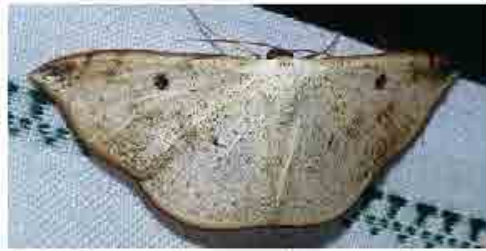

21. Somatina nr plynusaria

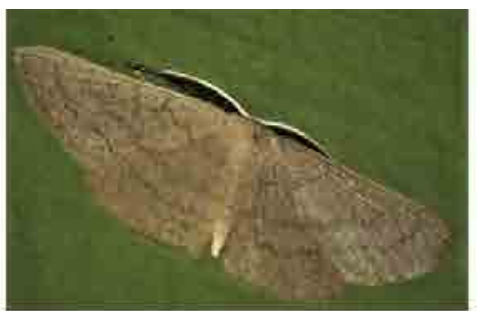

19. Scopula sp.

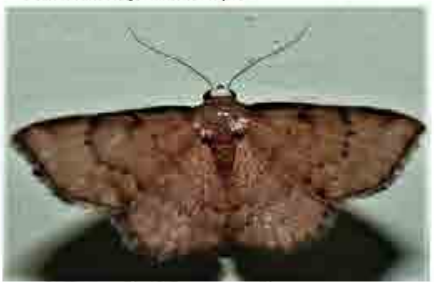

22. cf. Somatina.

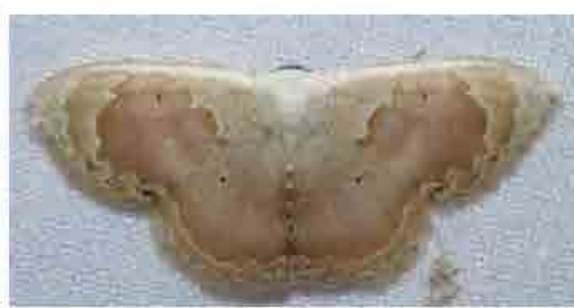

20. Somatina rosacea

Tribe Sterrhini: 23-32

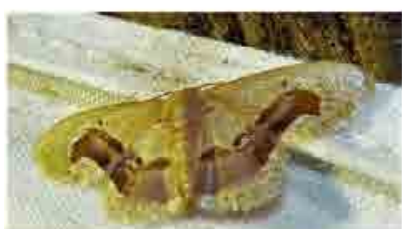

23. Idaea gemmaria

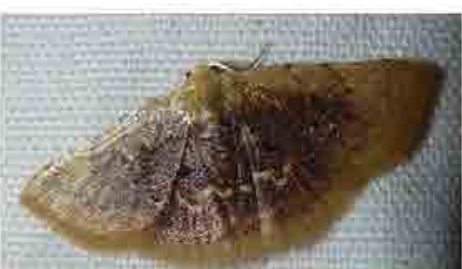

27. Idaea sp.5

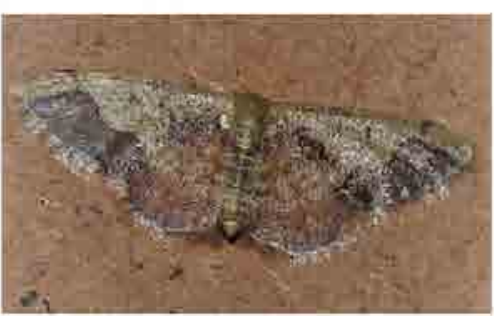

30. Idaea sp. 8

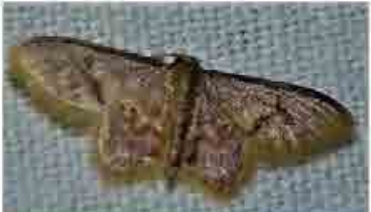

24. Idaea $\mathrm{nr}$ gemmaria

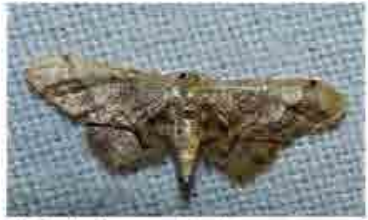

25. Idaea violaceae

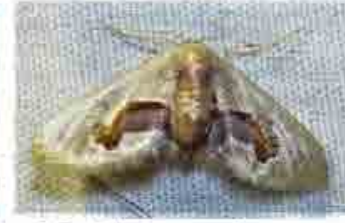

26. Idaea sp.4

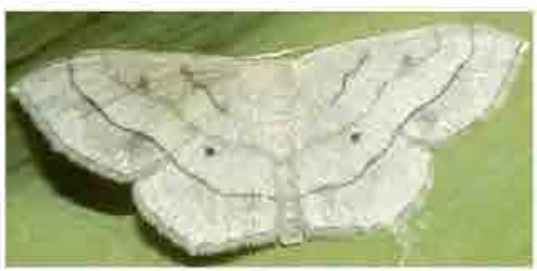

28. Idaea sp.6

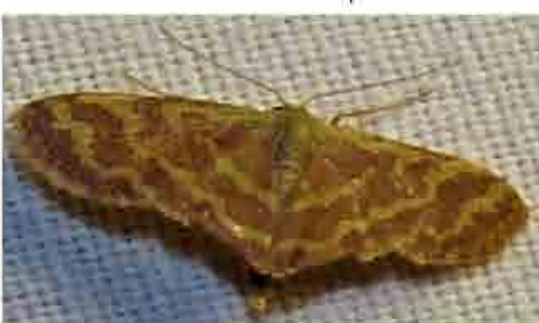

31. Lophophleps phoenicoptera

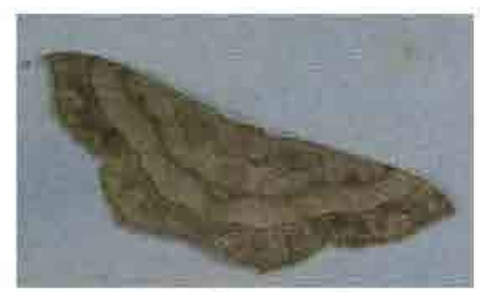

29. Idaea sp.7

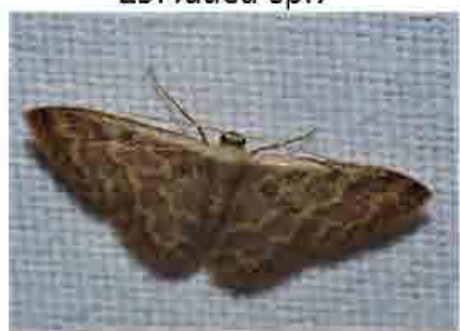

32. Lophophleps purpurea

Tribe: Timandrini: 33

Unidentified Sterrhinae: $34-36$

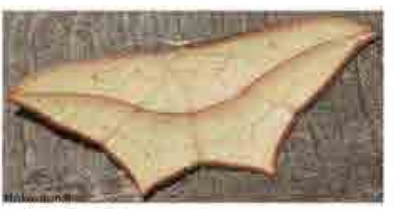

"33. Timandrasp.

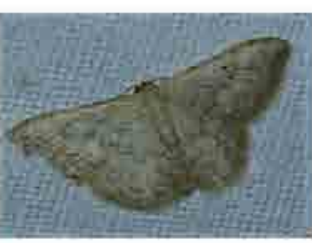

34.

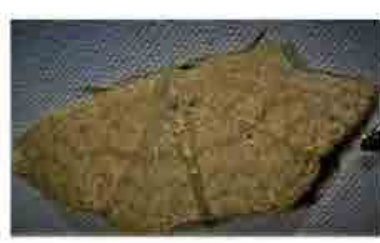

35.

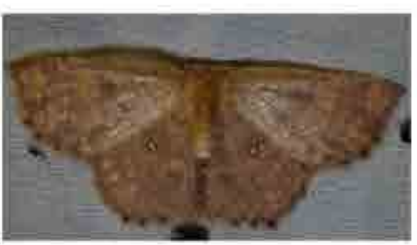

36.

Plate 2. Geometrids of Kalakad-Mundanthurai Tiger Reserve 
Subfamily Larentiinae: $37-60$

Tribe Asthenini: $37-38$

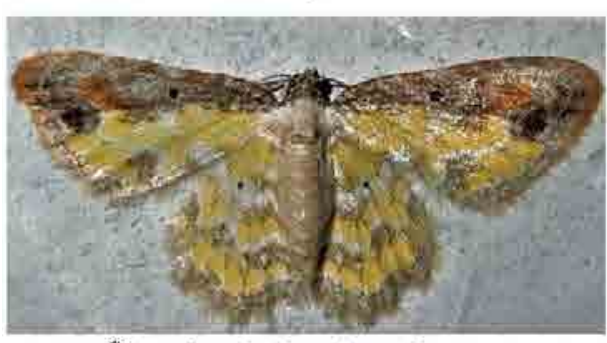

*37. Acolutha pictaria

Tribe Cidariini: $39-41$

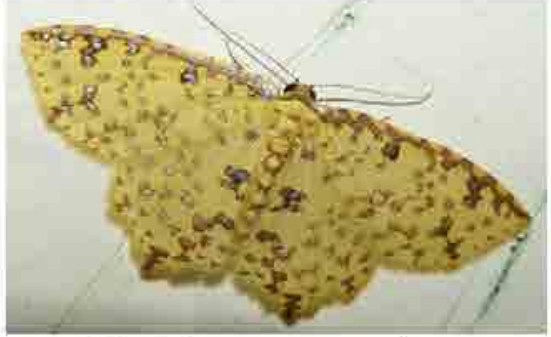

38. Polynesia sunandava

Tribe Eupitheciini: 42-53
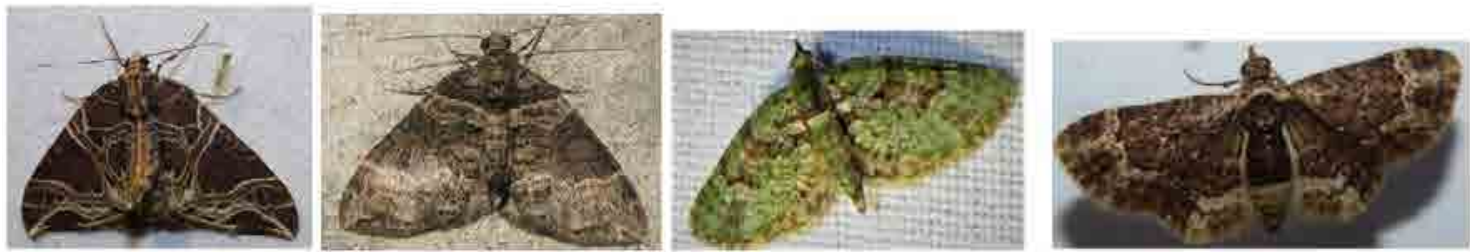

39. Ecliptopera dissecta ${ }^{*} 40$. Ecliptopera muscicolor 41. Chloroclystis sp. 42. Bosara albitornis

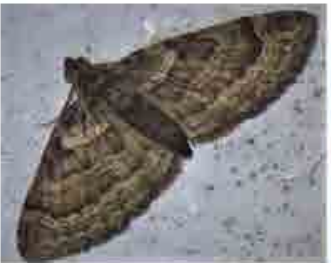

43. Eupitheciasp.

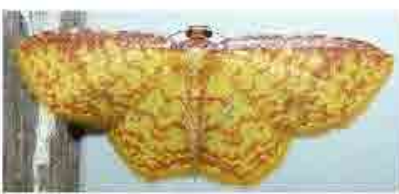

47. Eois cf. dissimilis

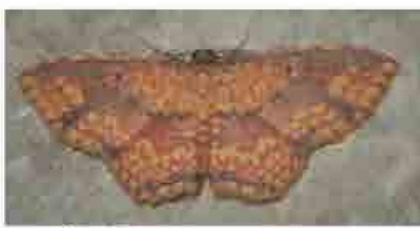

${ }^{*}$ 51. Eois sp. 4

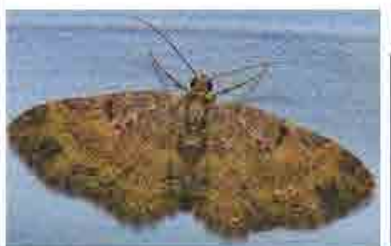

44. Collix sp.1

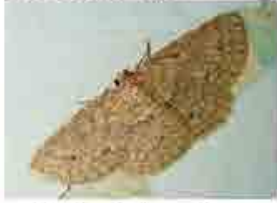

48. Eois lunulosa

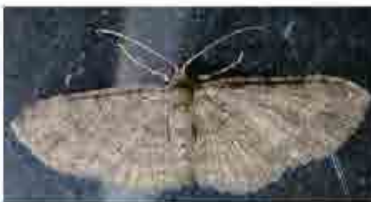

45. Collix sp. 2

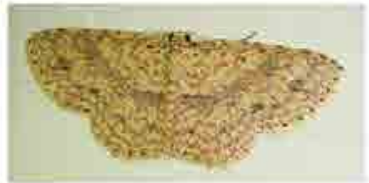

49. Eois lunulosa form ochraceae

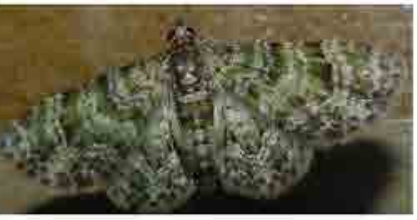

52. Gymnoscelis cf. admixtaria

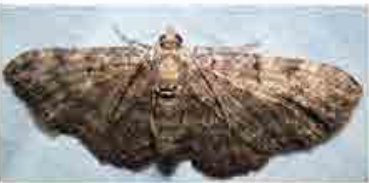

46. Collix sp. 3

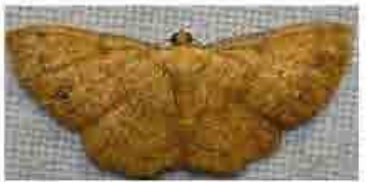

50. Eois sp. 3

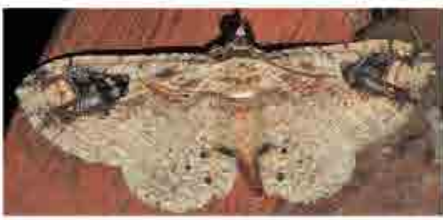

'53. Ziridava rubridisca
Tribe: Incertae sedis: 54

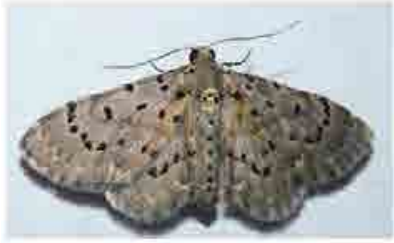

54. Physetobasis annulata
Tribe: Trichopterygini: 55-56

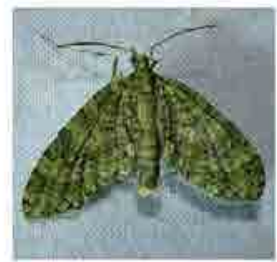

55. Sauris $\mathrm{sp} .1$

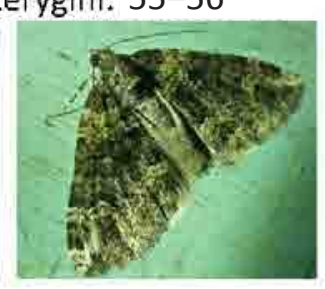

56. Sauris sp. 2
Tribe:Xanthorhoini: 57

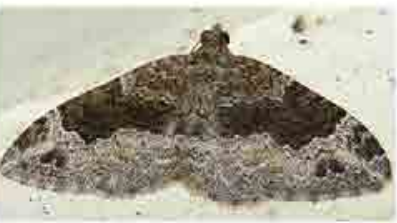

57. Xanthorhoe saturata

Plate 3. Geometrids of Kalakad-Mundanthurai Tiger Reserve 
Unidentified Eupitheciini: 58-60

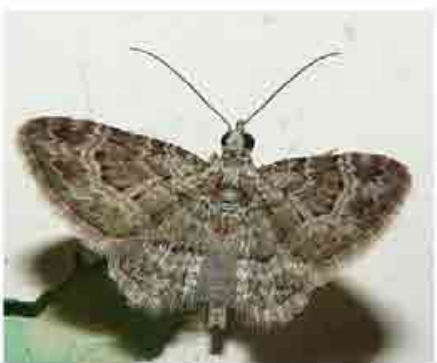

58.

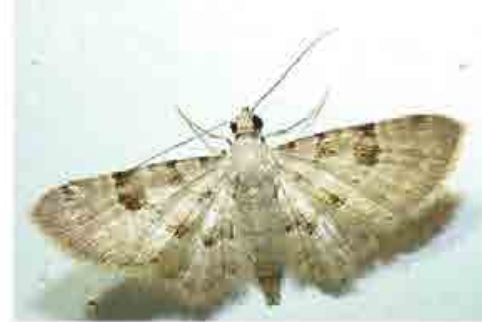

59.

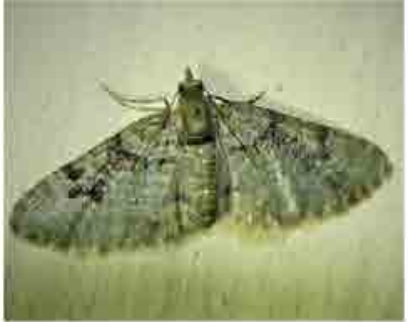

60.

Subfamily Geometrinae: 61-101

Tribe Agathiini: 61-63

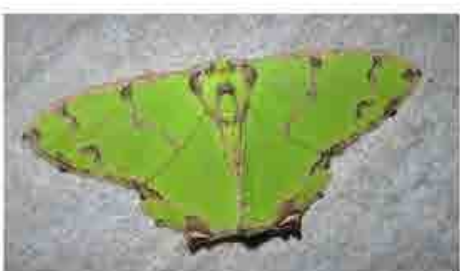

61. Agathia lycaenaria

Tribe Archaeobalbini: 64-65

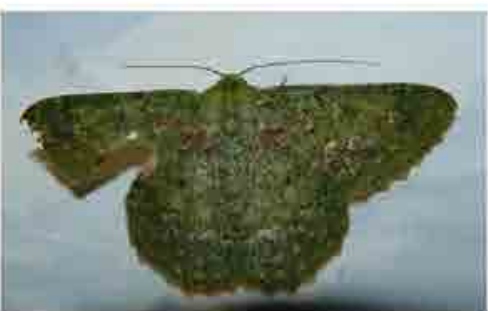

64. Herochroma cf. cristata

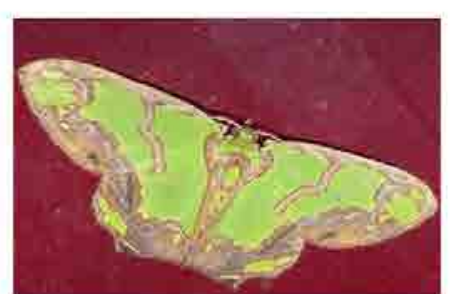

62. Agathia hemithearia

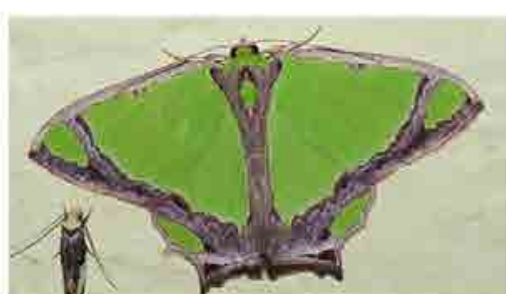

63. Agathia laetata

Tribe: Comibaenini: 66-74

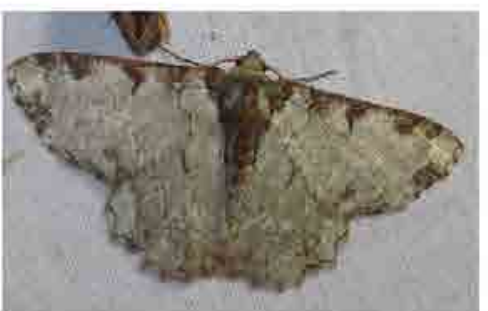

65. Lophophelma ruficosta

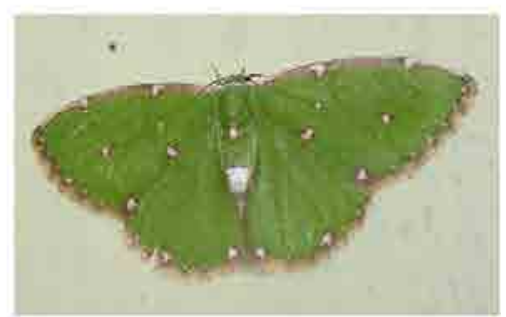

66. Argyrocosma inductaria

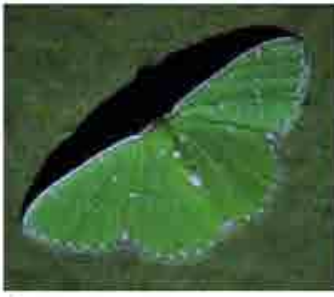

67.Chlorochromodes sp.

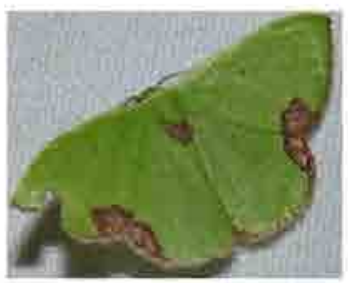

71. Comibaena integranota

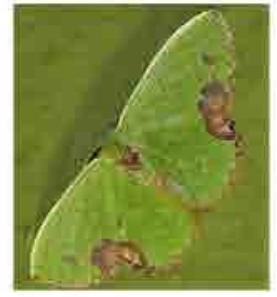

68. Comibaena attenuata

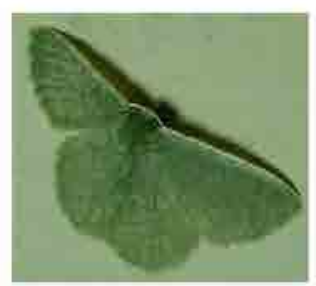

72. Comibaena cf. striataria

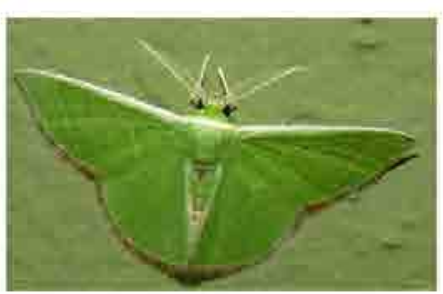

69. Comibaena cassidara

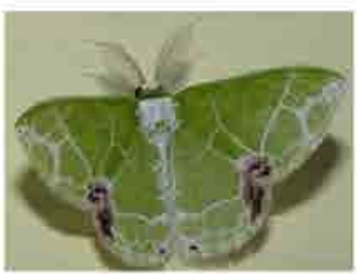

73. Protuliocnemis cf. biplagiato

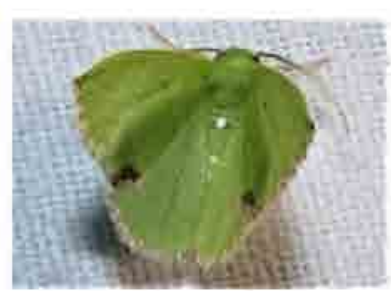

70. Comibaena fuscidorsata

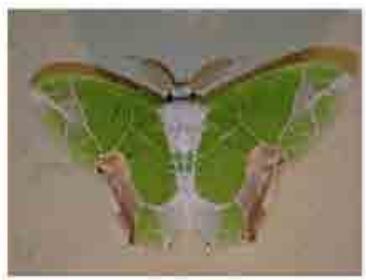

74. Protuliocnemis partita

Plate 4. Geometrids of Kalakad-Mundanthurai Tiger Reserve 
Tribe Dysphaniini: 75

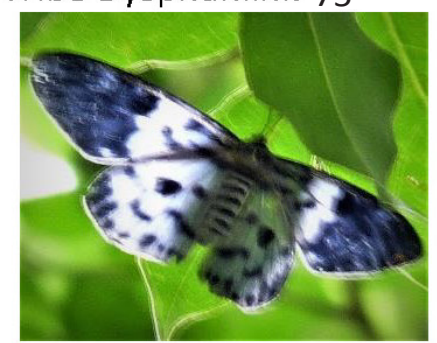

75. Dsyphania percota
Tribe Geometrini: 76

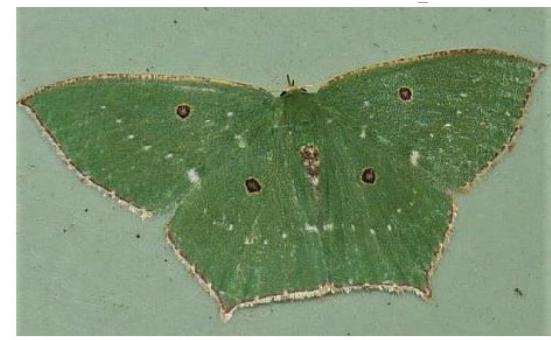

76. Cyclothea disjuncta
Tribe Hemitheini: 77-91

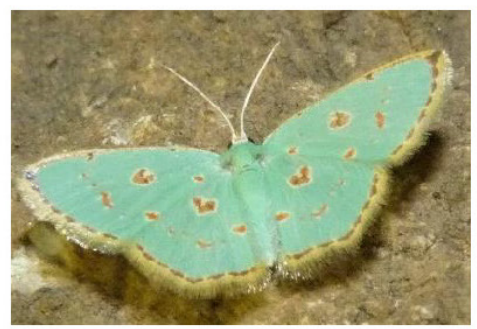

77. Comostola sp.

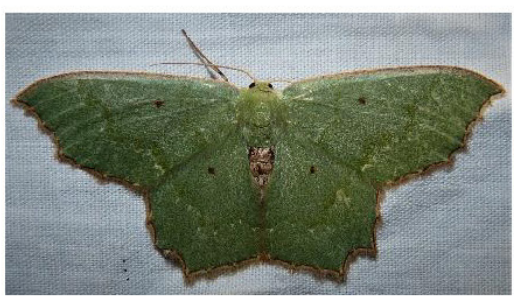

78. Episothalma robustaria

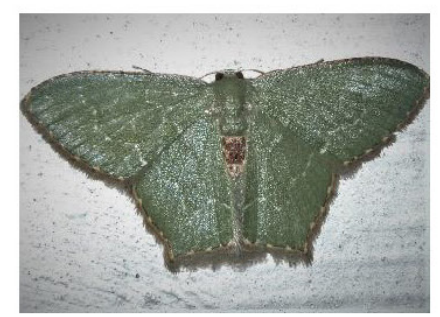

79. Hemithea tritonaria

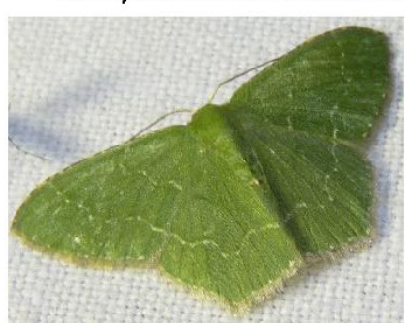

81. Idiochlora nr caudularia

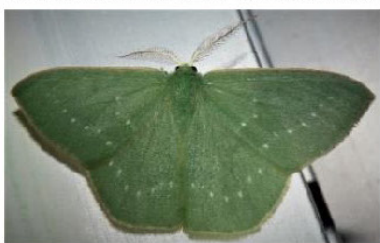

84. Pentheochlora cf. uniformis

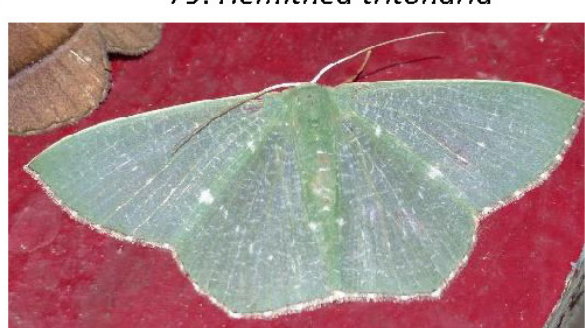

82. Orothalassodes hypocrites

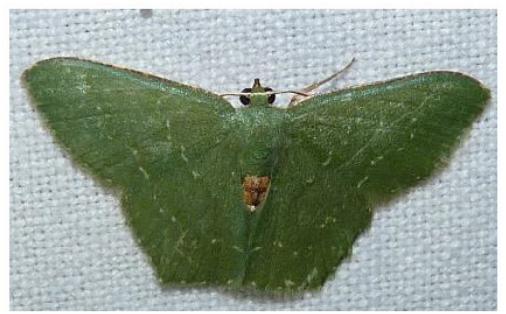

80. Hemithea wuka

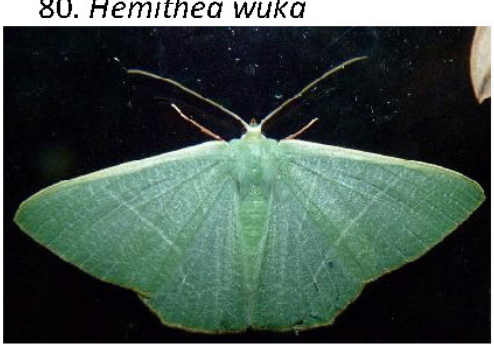

83. Pelagodes sp.

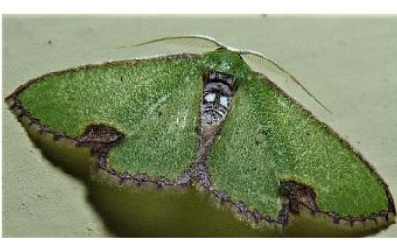

85. Spaniocentra sp.

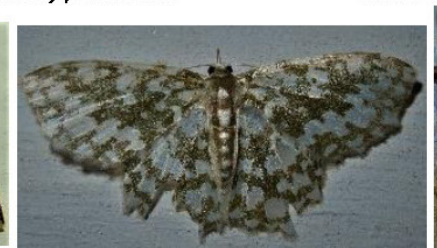

"86. Berta cf. chrysolineata

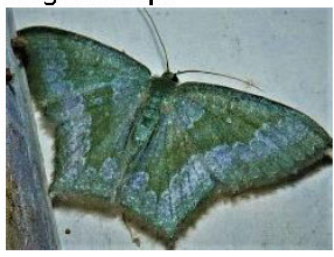

87. Jodis nr undularia

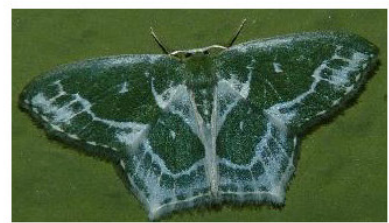

88. Jodis pallescens

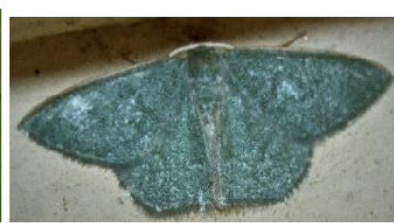

89. Jodis sp.

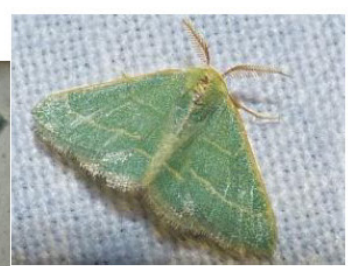

90. Microloxia indecretata

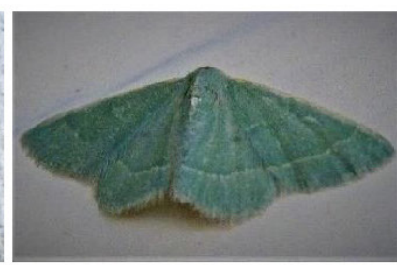

91. Microloxia sp.
Tribe Nemoriini: 92-93

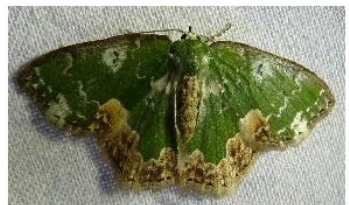

92. Eucyclodes albisparsa

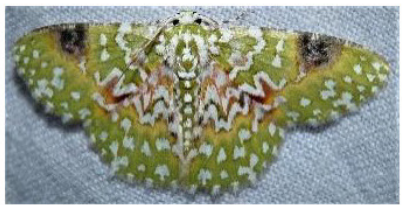

93. Eucyclodes gavissima

Plate 5. Geometrids of Kalakad-Mundanthurai Tiger Reserve

Tribe Ornithospilini: 95-96

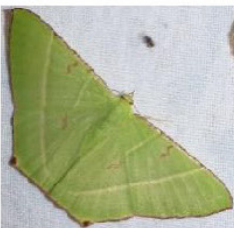

94. Ornithospila lineata

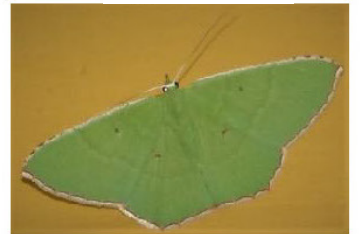

95. Ornithospila submonstrans 
Tribe Pseudoterpnini: 96-98

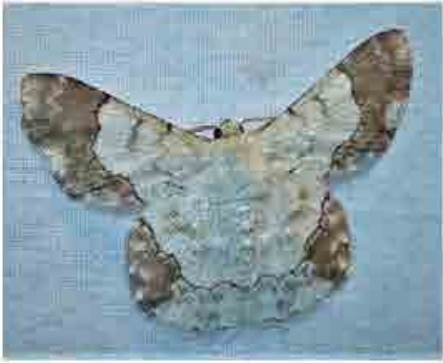

96. Pingasa ruginaria

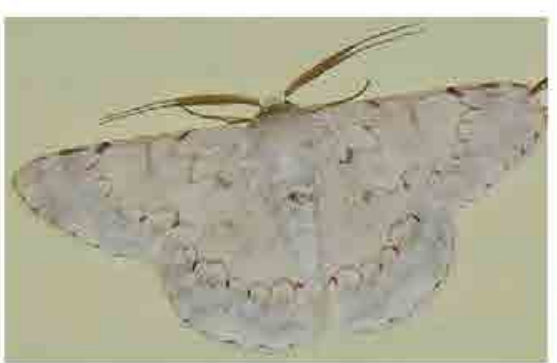

97. Pingasa dispensata ơ

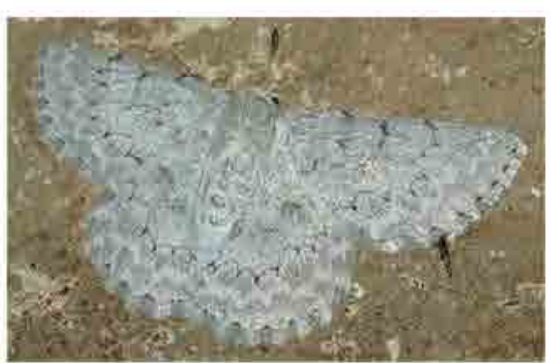

98. Pingasa dispensata $f$

Unidentified Geometrinae: 99-101

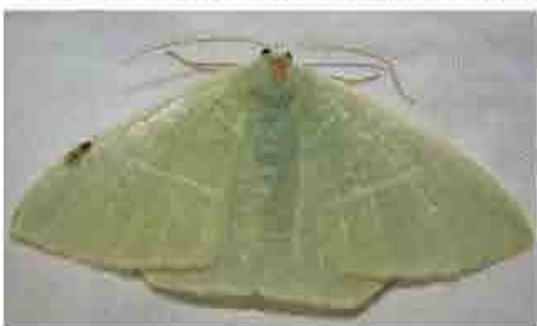

99.

Subfamily Desmobathrinae: 102-105

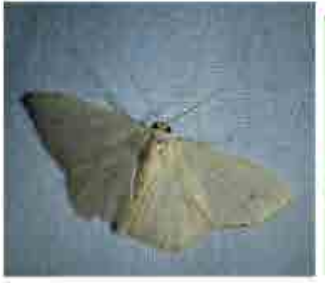

102. Derambila fragilis

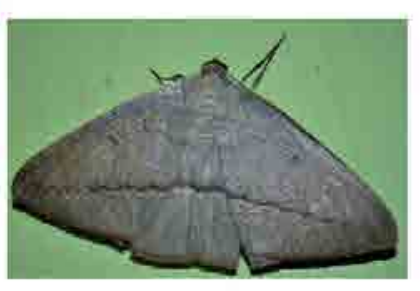

103. Noreia ajaia or

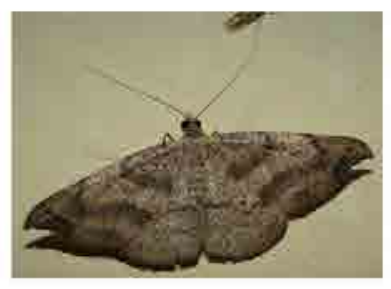

104. Ozola microniaria 우

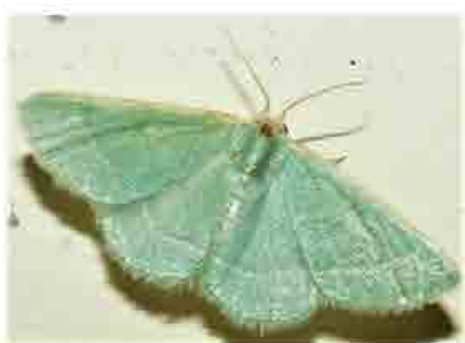

101.

Subfamily Ennominae: 106-274

Tribe Abraxini: 106-123

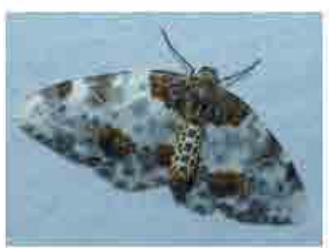

106. Abraxas leucostolo argyrosticta

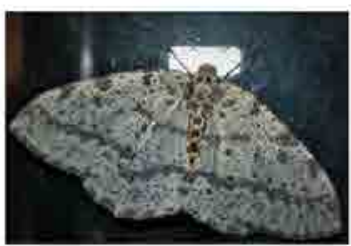

107. Abraxas fasciaria

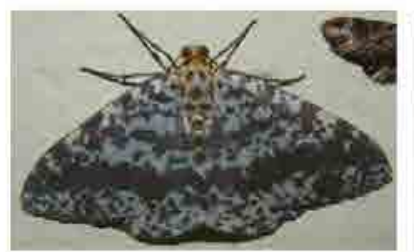

108. Abraxas poliostrota ơ 109.Abraxas poliostrota 우

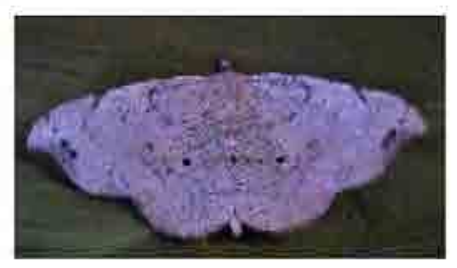

105. Ozola sp.
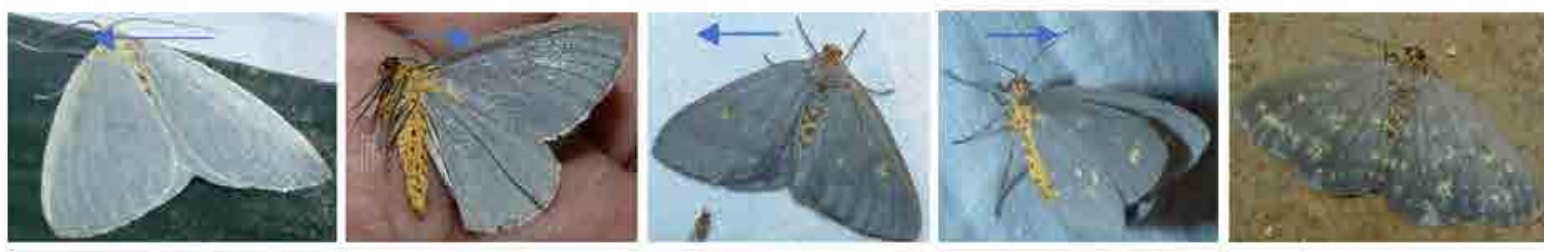

110

Abraxas sp.4 $\rightarrow$ 111.(UN) 112

4

Plate 6. Geometrids of Kalakad-Mundanthurai Tiger Reserve 

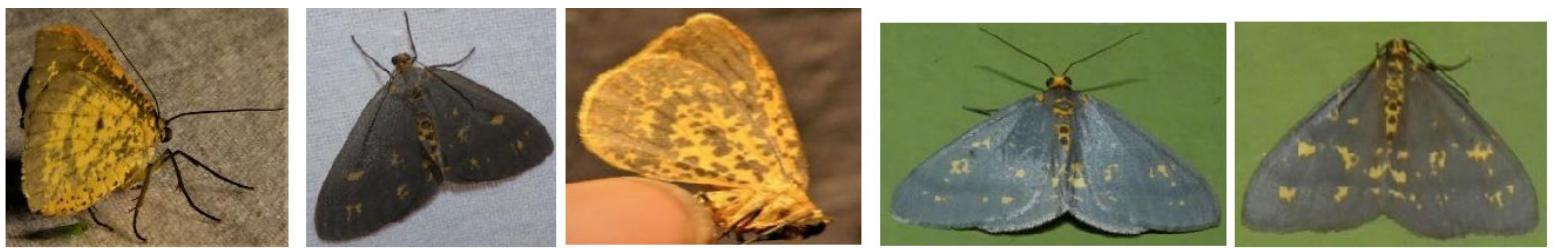

115. Abraxas sp.6 (UN) 116. (UP) $\longleftarrow$ Abraxas sp. $7 \longrightarrow$ 117.(UN) 118.Abraxs sp. /form 119. Abraxas sp./form
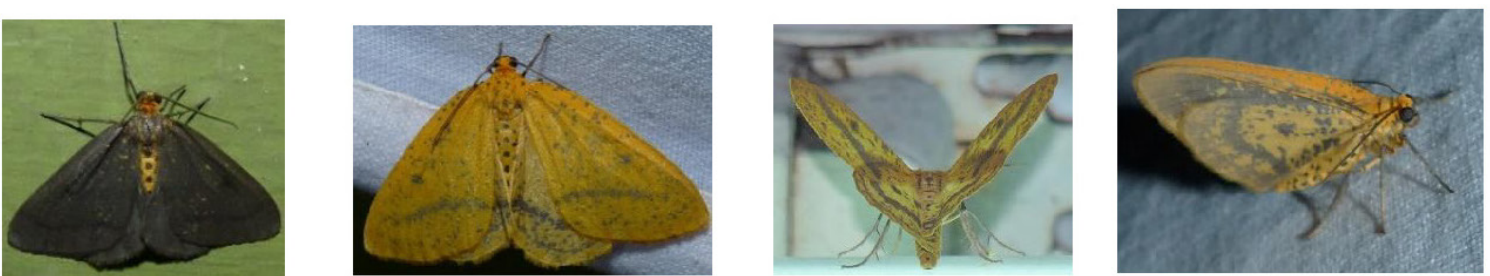

120. Abraxas sp./form

121.

Abraxas sp. 8.(UP)

122. 123. (UN) sp.8

Tribe Baptini: 124-129

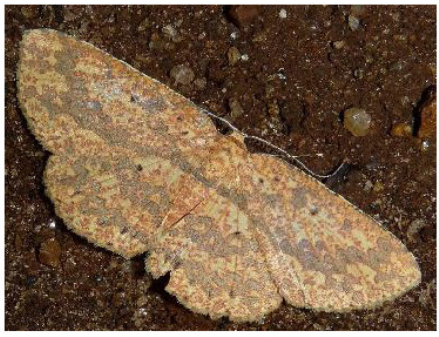

124. Borbacha cf. pardaria

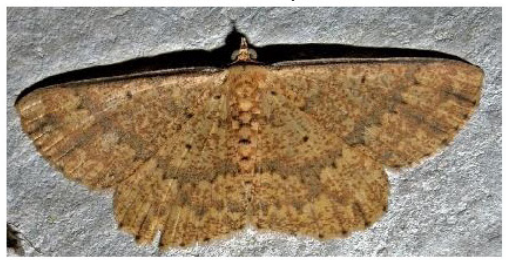

*127. Synegia imitaria

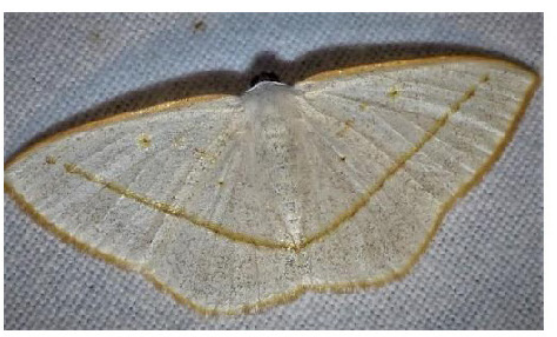

125. Lomographa inamata

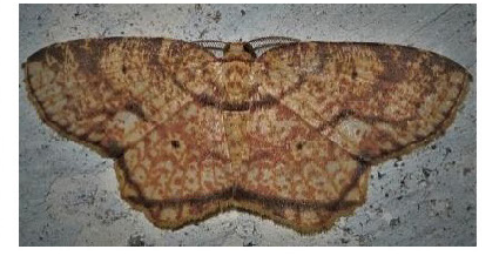

${ }^{*} 128$. Yashmakia erythra o

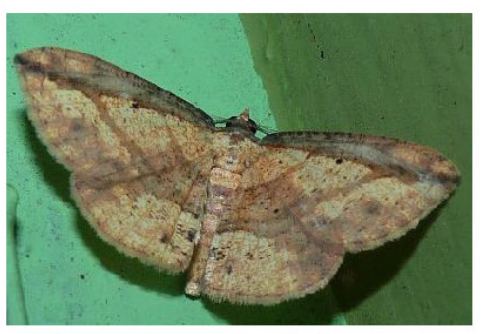

126. Platycerota vitticostata

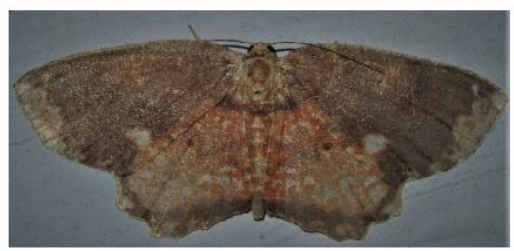

*129. Yashmakia conflagrata?

Tribe Boarmiini: 130-190
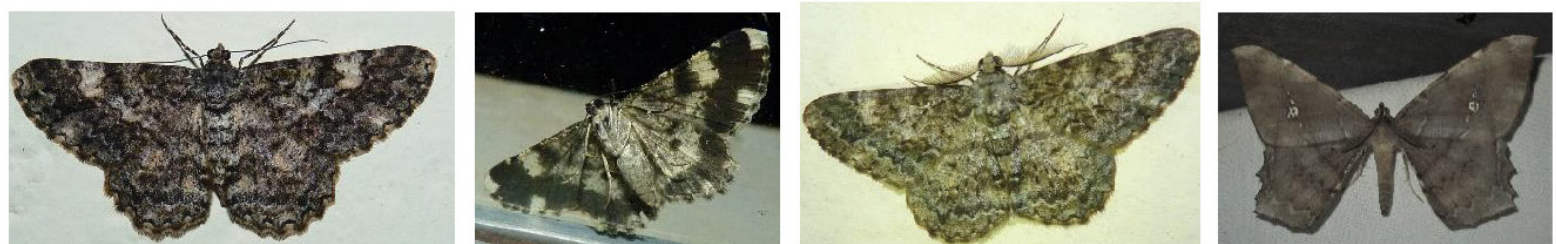

130. Alcis nilgirica + -UP

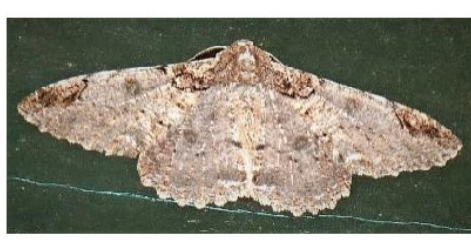

134. Amraica recursaria

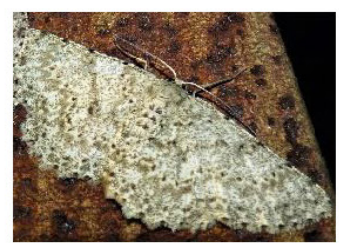

135. Catoria cf. sublavaria + UP \& 136. UN

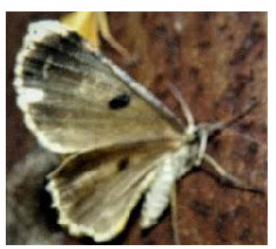

Plate 7. Geometrids of Kalakad-Mundanthurai Tiger Reserve

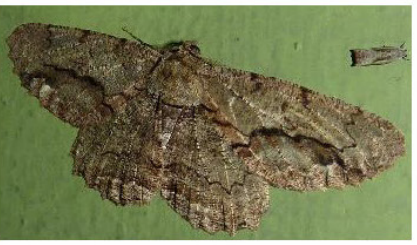

137. Chorodna strixaria 


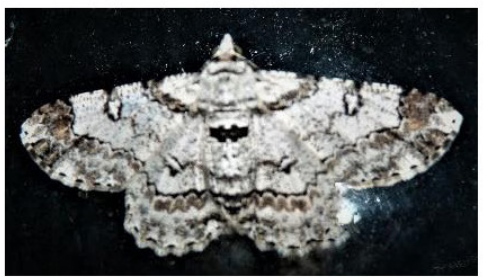

138. Cleora alienaria +U-UP

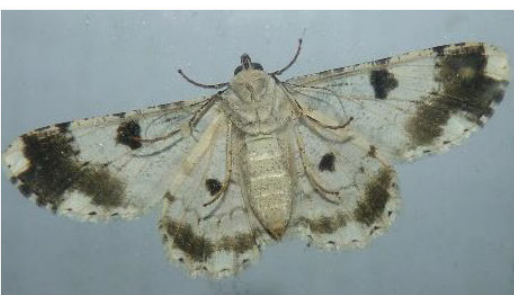

139. Cleora alienaria 9 - UN

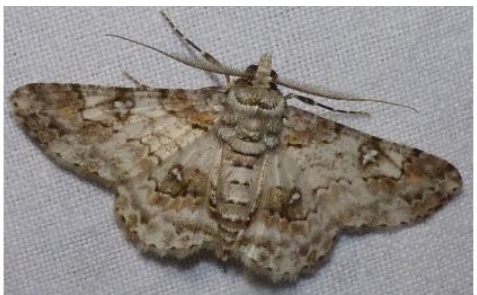

140. Cleora alienaria $\hat{\text { ? }}$

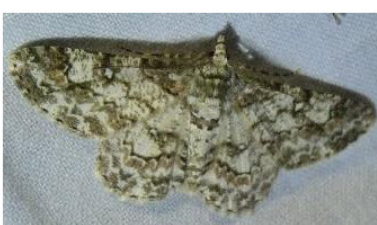

141. Typical form

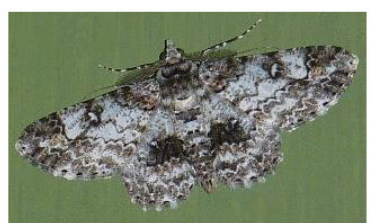

142. Variant

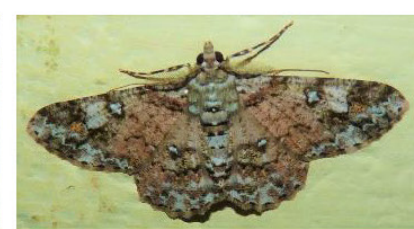

143. Colour variant

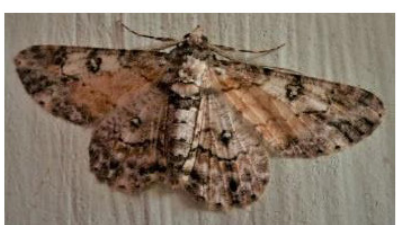

144. Colour variant Cleora alienaria 0

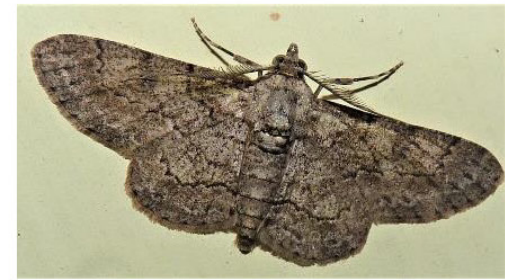

145. Cleora $\mathrm{nr}$ injectaria

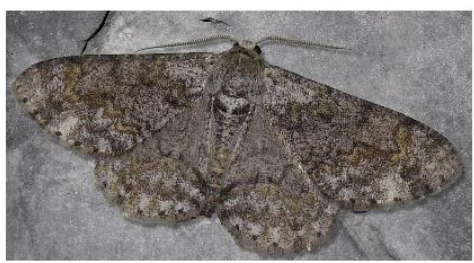

148.Cusiala boarmoides

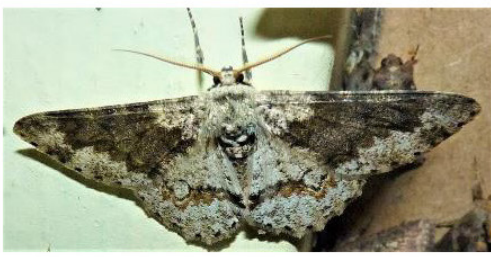

151. Cusiala raptaria, form disterminata -variant

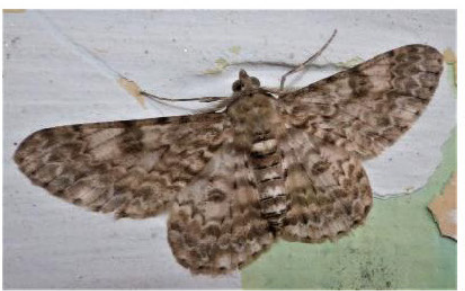

146. Cleora sp. 3

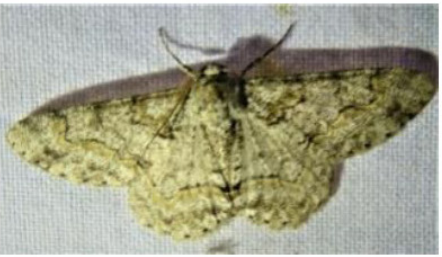

149. Cusiala raptaria

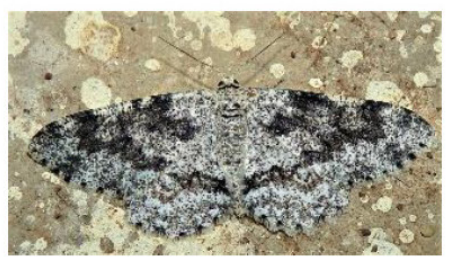

152. Cusiala raptaria, form disterminata - variant

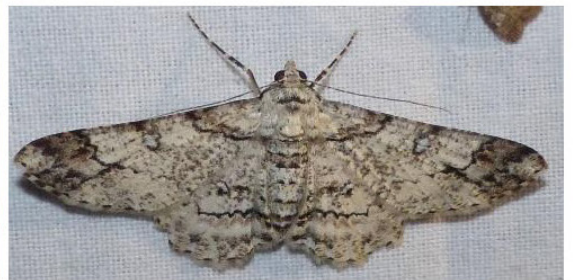

147. Ascotis cf. imparata 9

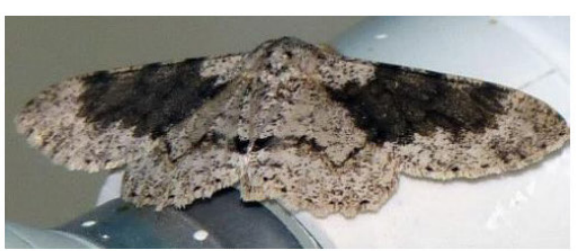

150. Cusiala raptaria form disterminata

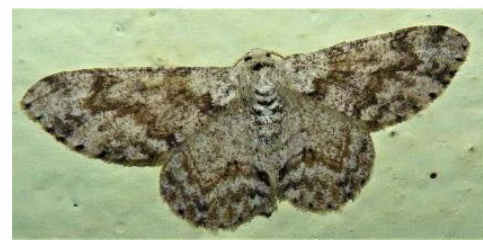

153. Cusiala raptaria, form rufifasciata

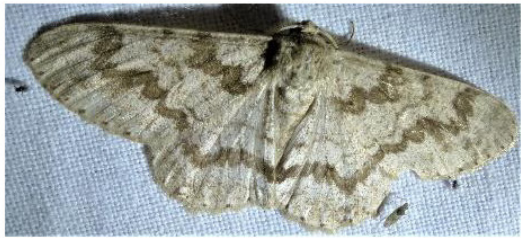

154. C raptaria, form suiasasa

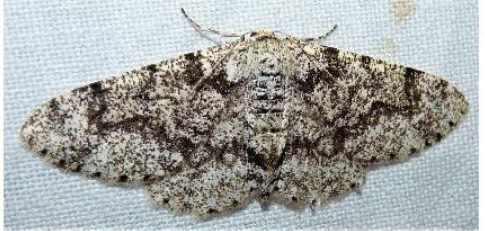

155.

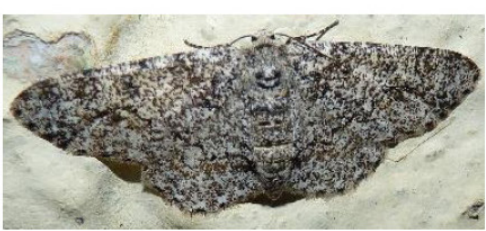

156.

Plate 8. Geometrids of Kalakad-Mundanthurai Tiger Reserve 


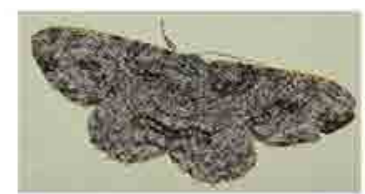

157.

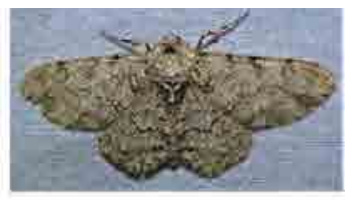

158.

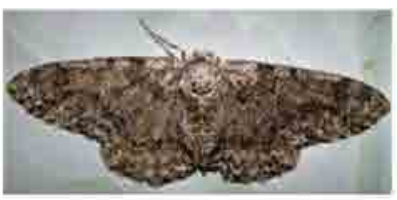

159.

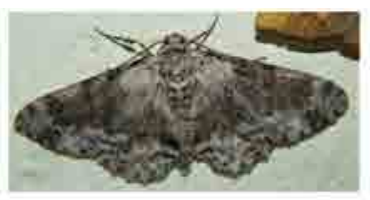

160.

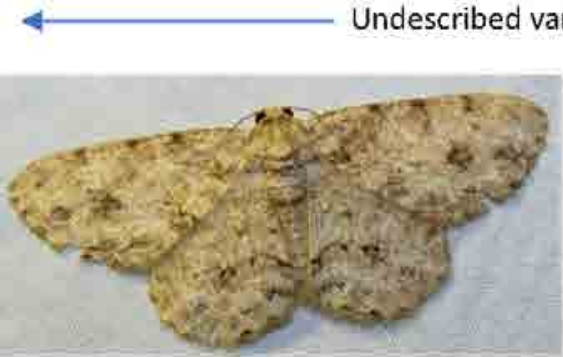

161. Ectropis bhurmitra

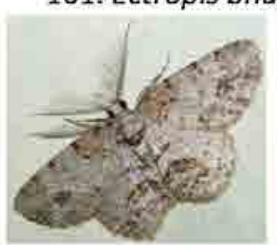

164. 8

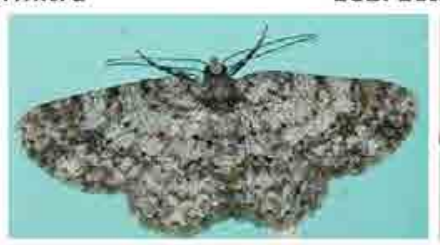

165. 9
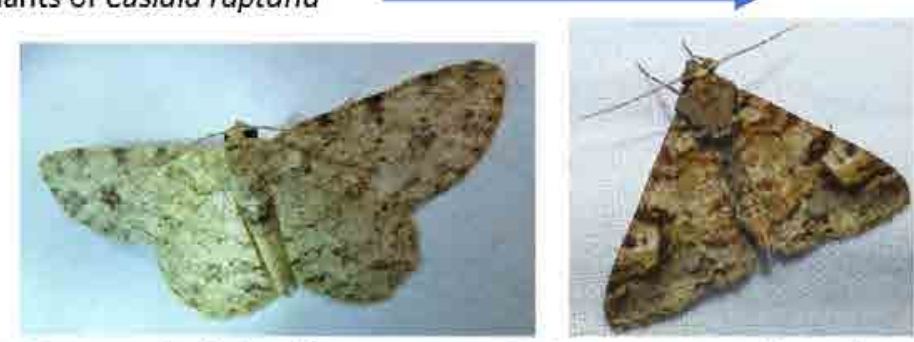

163. Gasterocome polyspathes

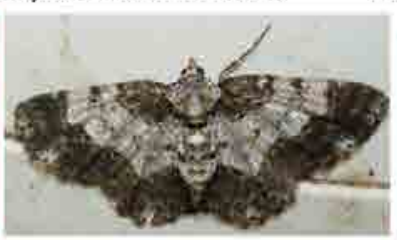

166. 8

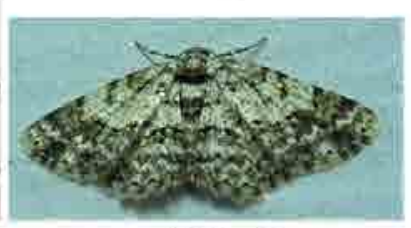

167. ㅇ
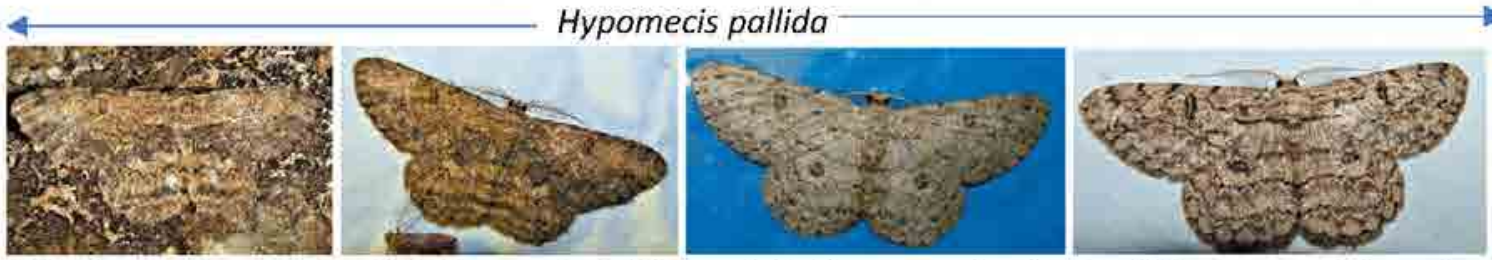

168. Hypomecis separata 169. H separata, dark form 170. Hypomecis tamilensis 171. Hypomecis transcissa

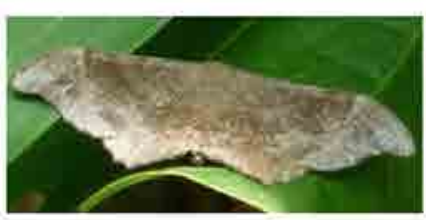

172. Hyposidra talaca

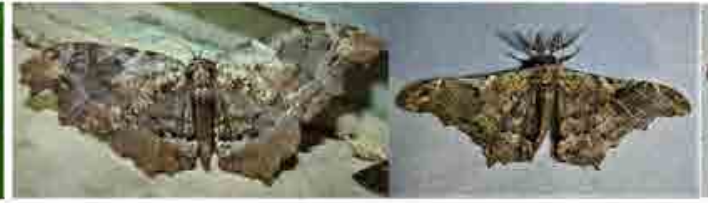

173. Hyposidra violescens of $174 . \mathrm{H}$ violescens 0

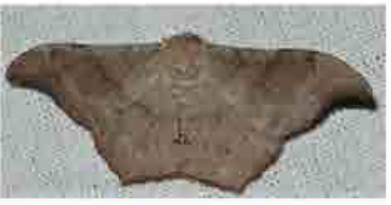

175. Hyposidra sp.

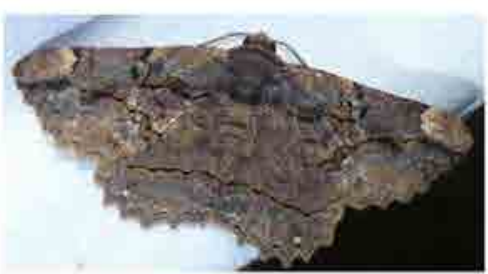

176. Dasyboarmia cf. inouei

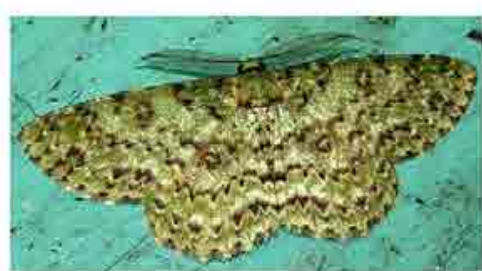

177. Ophthalmitis cf. herbidaria

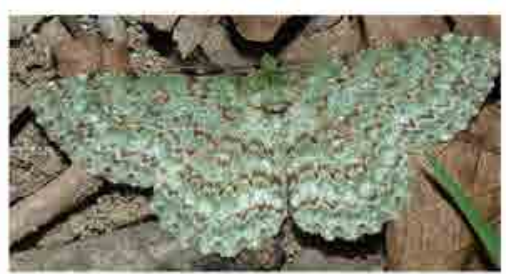

178. Ophthalmitis kalakadaria

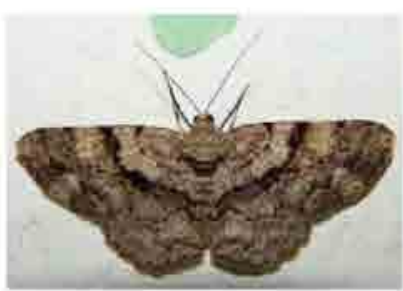

179. Psilalcis cf. subtochracea

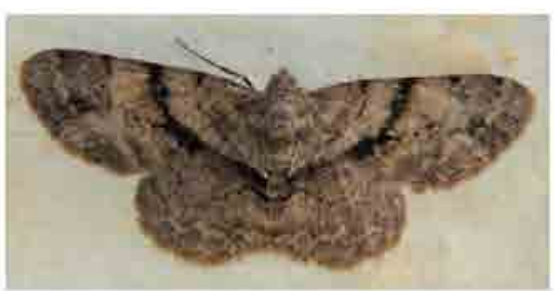

180. Psilalcis cf. subtochracea 8

Plate 9. Geometrids of Kalakad-Mundanthurai Tiger Reserve

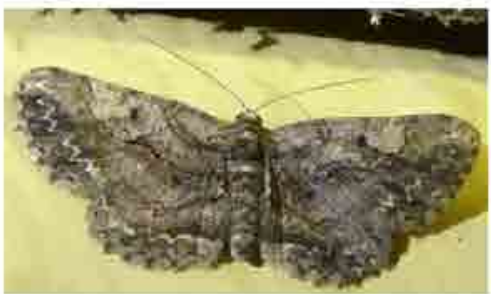

181. Psilalcis sp. 2 


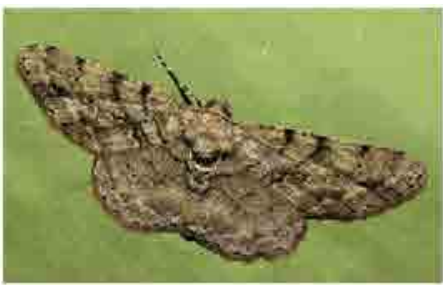

182. Psilalcis sp.3?

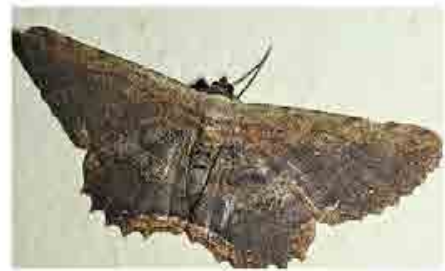

185. Ruttellerona cf. cessaria

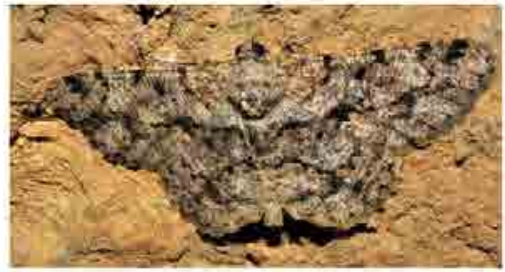

183. Psilalcis sp. 4 ह

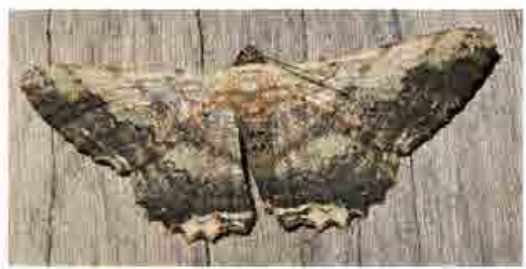

"186. Ruttellerona cf. pseudocessaria

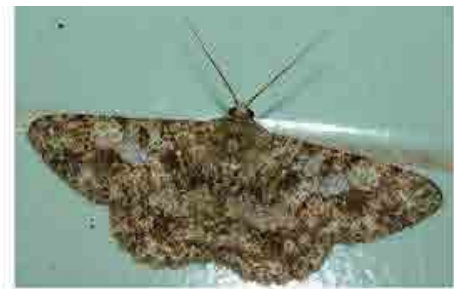

184. Racotis keralaria

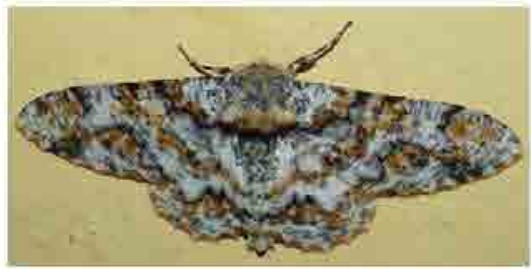

187. Biston strigaria
Unidentified Boarmiini: 188-190

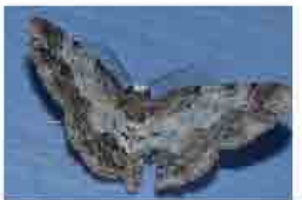

188.

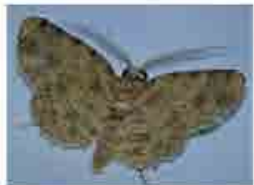

189.

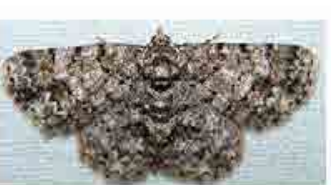

190.

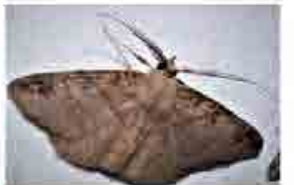

191. Petelia medardaria

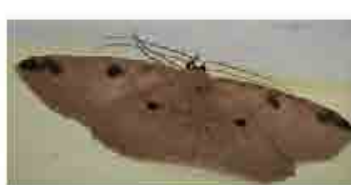

192. Petelia distracta우

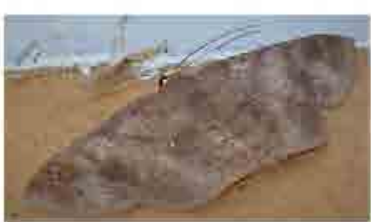

193. Petelia fasciata

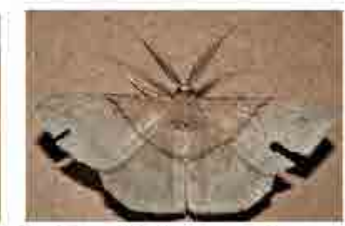

194. Petelia immaculata

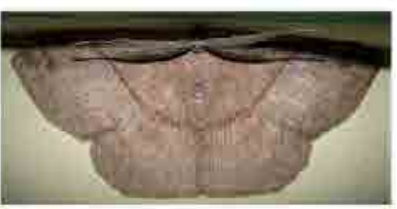

195. Petelia immaculata 3

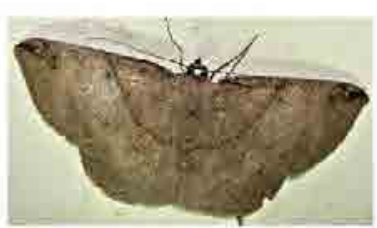

196. Petelia immaculata 9

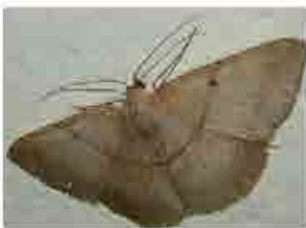

197. Petelia immaculata 우

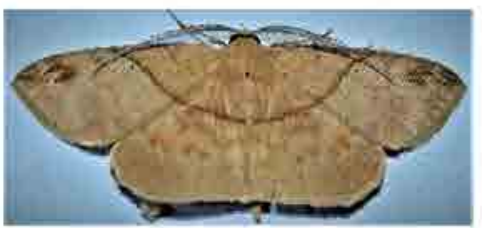

198. Petelia sp.5

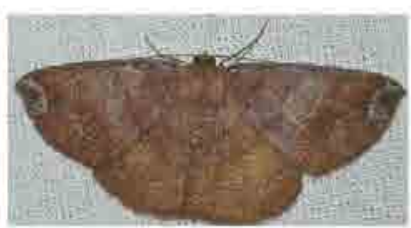

*199. Astygisa sp.

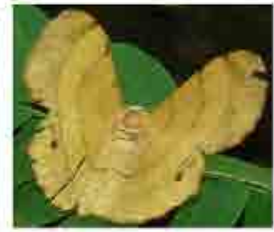

200. Hyperythra lutea

Tribe Cassymini: 201-206

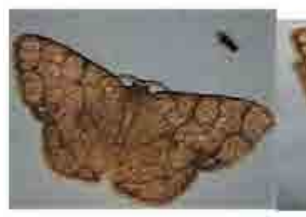

201. Heterostegane subtessellata 8

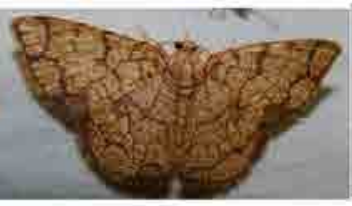

202. Heterostegane subtessellata?

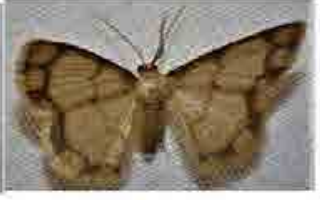

203. Heterostegane cf. tritocampsis ?

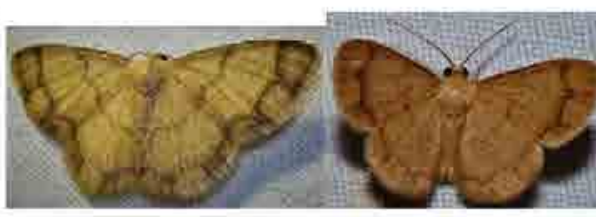

204. Heterostegane 205. Heterostegane cf. tritocampsis ô

sp. 3

Plate 10. Geometrids of Kalakad-Mundanthurai Tiger Reserve 
Tribe: Ennomini: 207-209

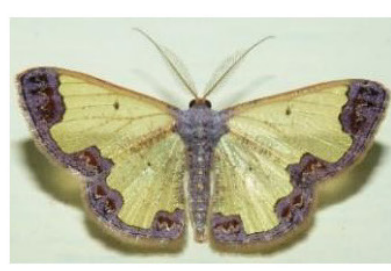

206. Zamarada cf. excisa

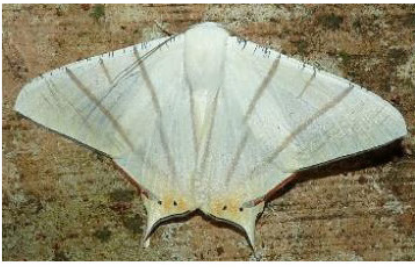

207. Ourapteryx marginata
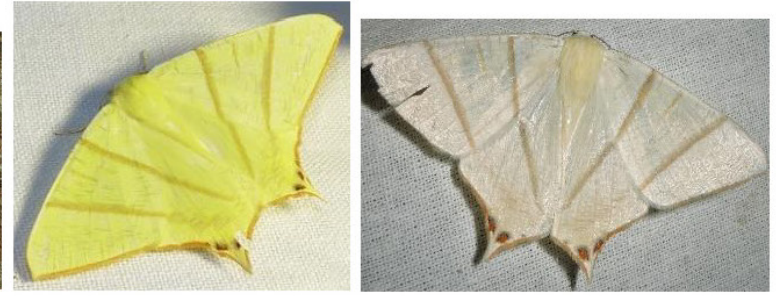

208. Ourapteryx peermaadiata $\longrightarrow 209$. $^{*}$

Tribe Eutoeini: 210-219

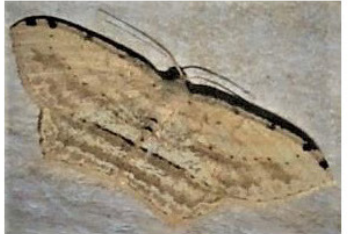

*210. Calletaera postvittata

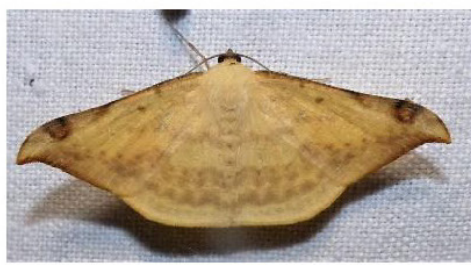

214. Luxiaria phyllosaria

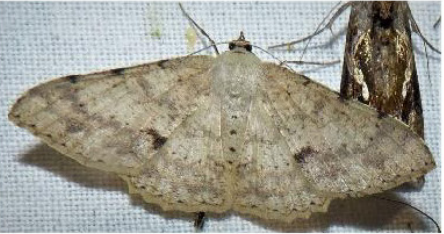

211. Luxiaria emphatica

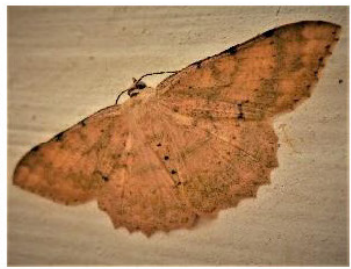

215. Luxiaria sp.4

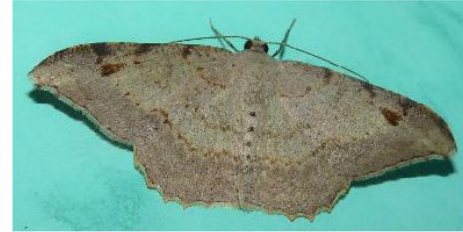

212. $\longleftarrow$ Luxiaria hypaphanes $\longrightarrow 213^{*}$.

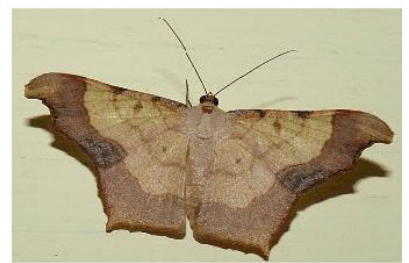

216. Zeheba $\mathrm{nr}$ aureata 9 217. Zeheba $\mathrm{nr}$ aureata

Tribe Gonodontini: 220

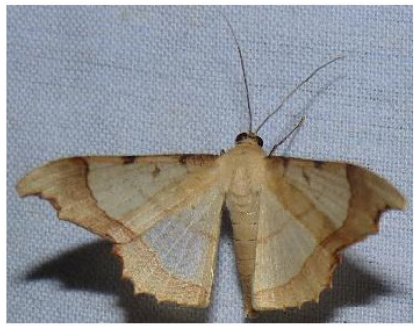

218. Zeheba nr aureata

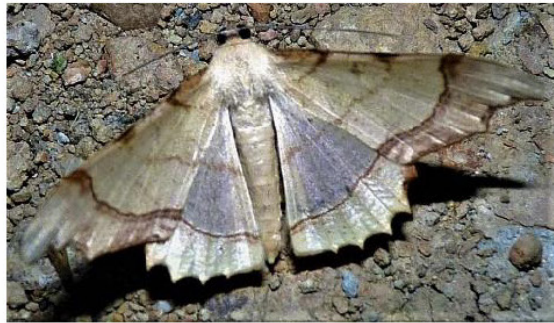

219. Zeheba cf. aureatoides

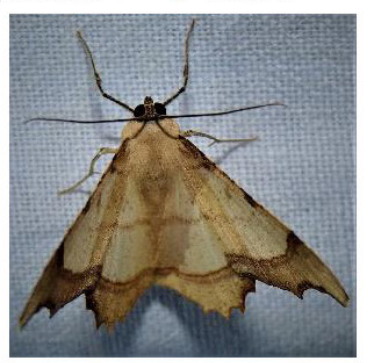

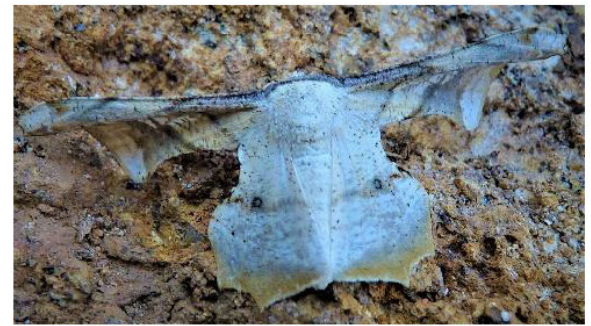

220. Gonodontis pallida

Tribe Hypochrosini: 221-246

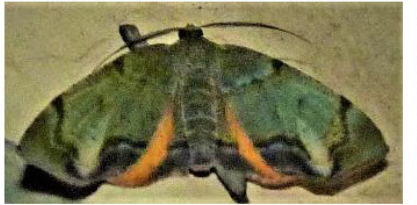

221. $\leftarrow$ Achrosis incitata complex $\longrightarrow 222^{*}$.

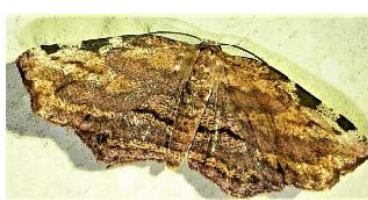

223. Achrosis intexta

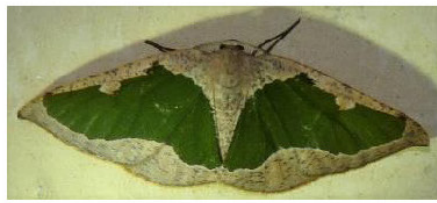

224. Celenna festivaria form formosensis

Plate 11. Geometrids of Kalakad-Mundanthurai Tiger Reserve 


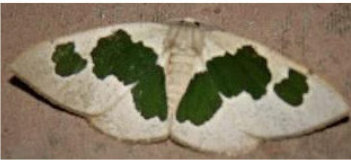

225. Celenna festivaria

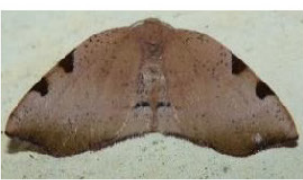

226

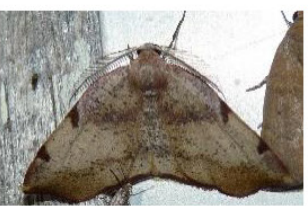

Achrosis sp. 48

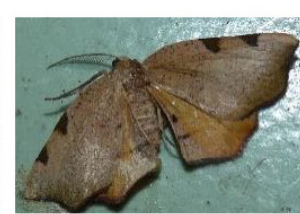

228.

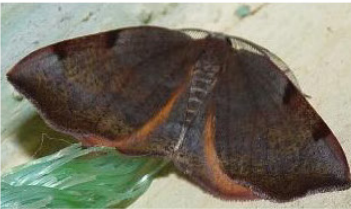

230. Achrosis sp. 4 ?

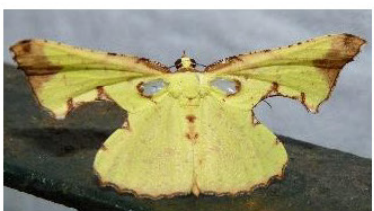

231. Corymica sp.

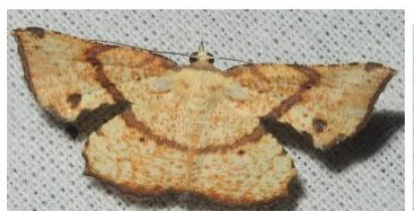

${ }^{*}$ 232. Corymica deducta

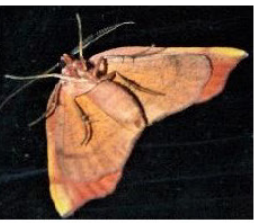

229 .

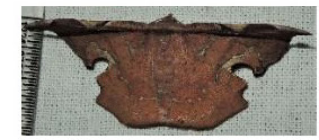

234. 9

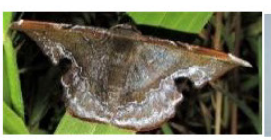

235. ठै

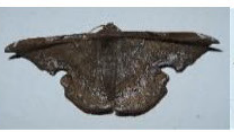

236. 0

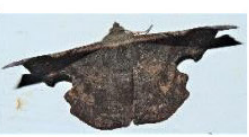

237. 0

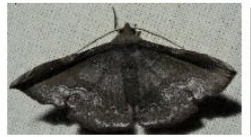

238(a). $\lesssim$

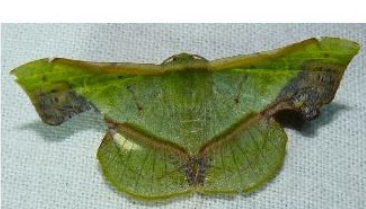

233. Fascellina plagiata
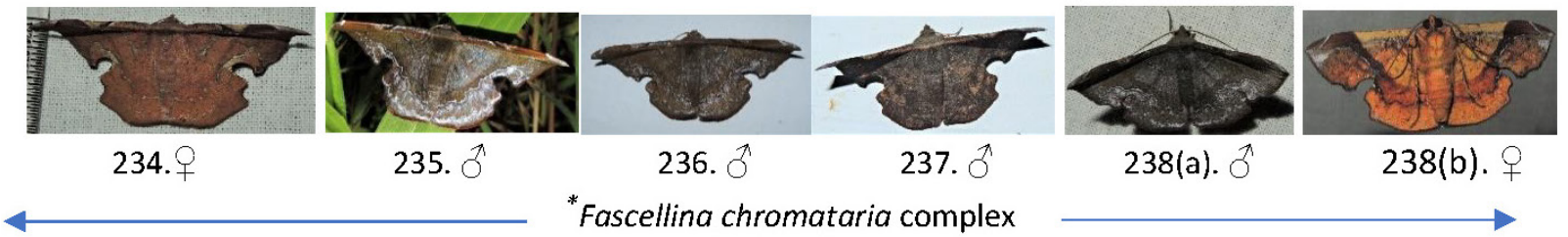

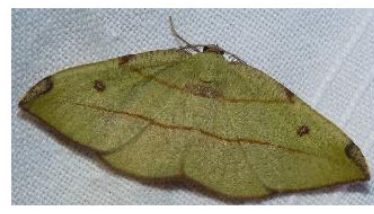

239.

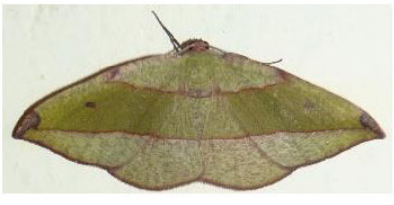

240.

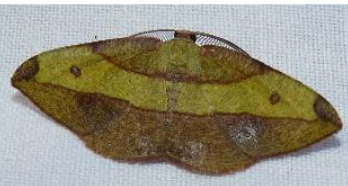

241.

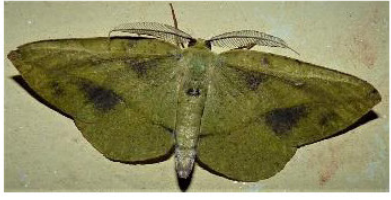

242. Omiza miliaria $\widehat{\delta}$

1 Hypochrosis hyadaria-Hypochrosis chlorozonaria

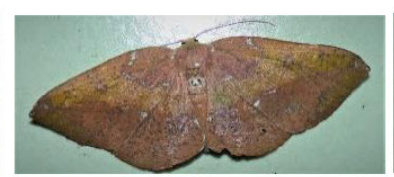

245. Omiza miliaria $?$

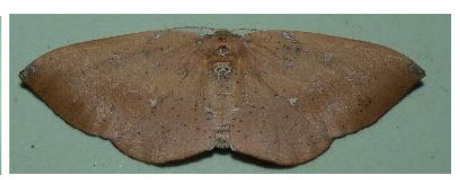

246. Omiza miliaria 9

Tribe Incertae sedis: $247-249$

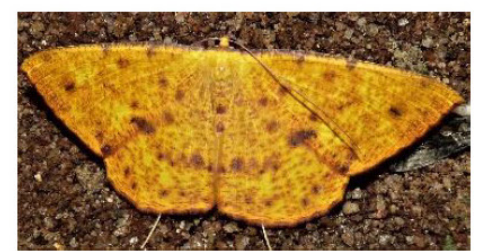

247. Eumelea ludovicata

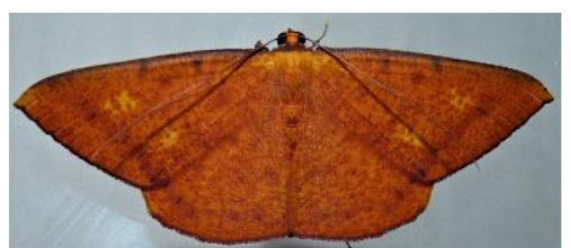

248. (UP)

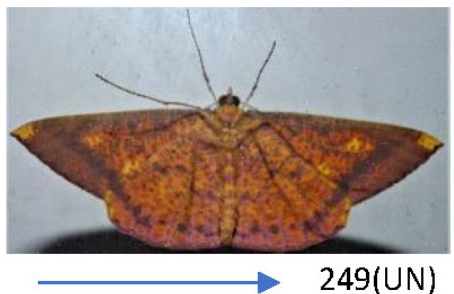

Eumelea sp. 2

249(UN)

Tribe Macariini: 250-269

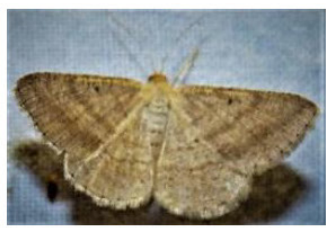

250. Acanthovalva sp.

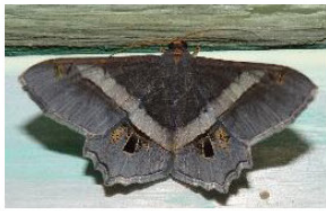

251.

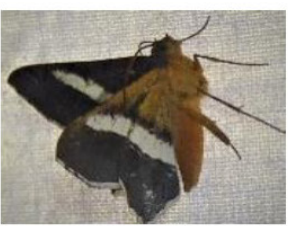

252 .

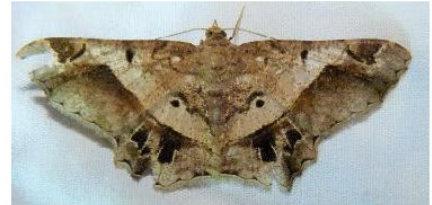

253. Chiasmia myandaria

Plate 12. Geometrids of Kalakad-Mundanthurai Tiger Reserve 


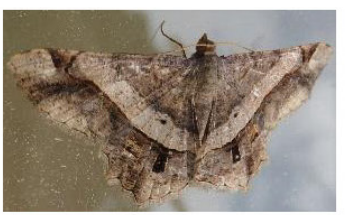

254.Chiasmia inchoata(UP) 255. C.inchoata(UN)

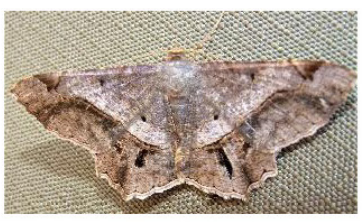

256. C.inchoata-variant

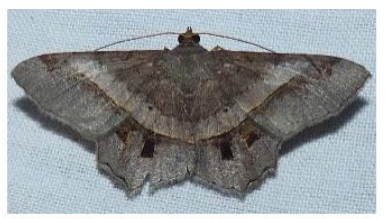

257. Chiasmia nora

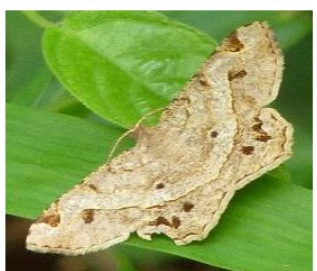

261. Chiasmia triangulata

258. Chiasmia ornataria

*259. Chiasmia ozararia

260. Chiasmia perfusaria

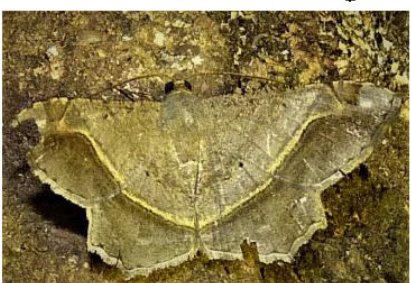

262.Chiasmia cf. normata 263.Chiasmia sp.11

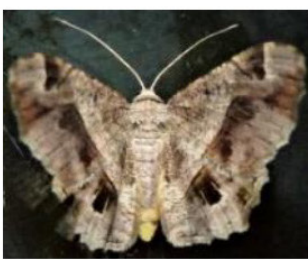

264. Chiasmia sp. 12

265. Chiasmia sp. 13

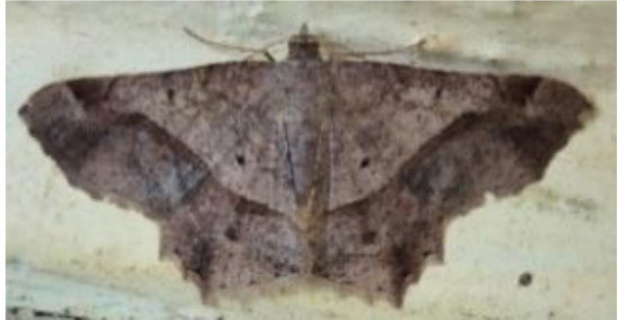

266. Chiasmia sp. 14

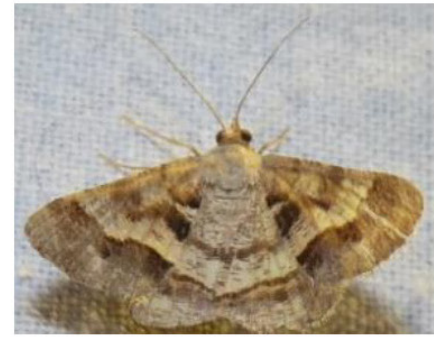

269. Isturgia disputaria

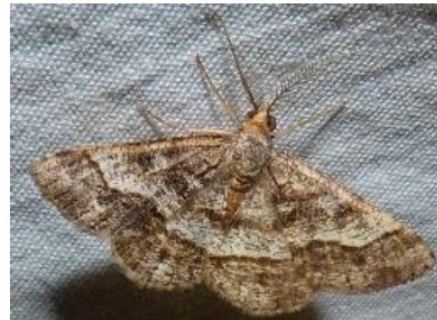

267. 8 Isturgia disputaria group

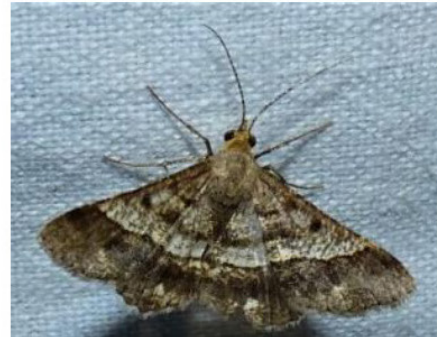

268. ?

Tribe Plutodini: 270-271

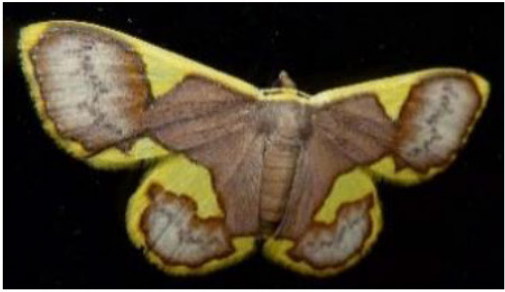

270. Plutodes nilgirica

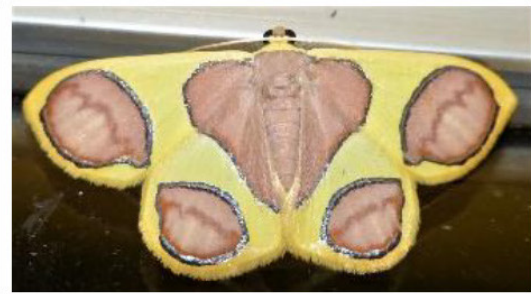

271. Plutodes pseudocyclaria

Tribe Scardamiini: $272-274$

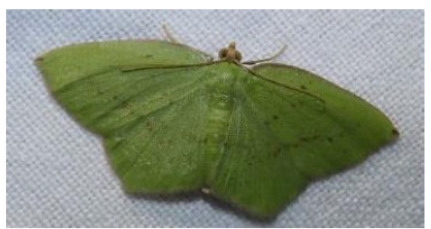

272.

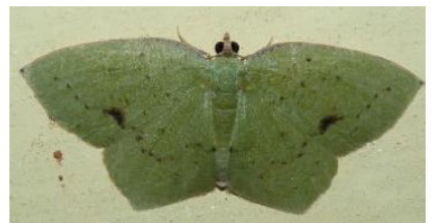

273.

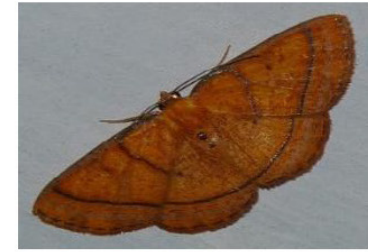

274. Scardamia metallaria

Plate 13. Geometrids of Kalakad-Mundanthurai Tiger Reserve. @ I Images with * are contributions from Thalavaipandi. All others are those of the first author. 
Dr. John Noyes, Natural History Museum, London, UK

Dr. Albert G. Orr, Griffith University, Nathan, Australia

Dr. Sameer Padhye, Katholieke Universiteit Leuven, Belgium

Dr. Nancy van der Poorten, Toronto, Canada

Dr. Kareen Schnabel, NIWA, Wellington, New Zealand

Dr. R.M. Sharma, (Retd.) Scientist, Zoological Survey of India, Pune, India

Dr. Manju Siliwal, WILD, Coimbatore, Tamil Nadu, India

Dr. G.P. Sinha, Botanical Survey of India, Allahabad, India

Dr. K.A. Subramanian, Zoological Survey of India, New Alipore, Kolkata, India

Dr. P.M. Sureshan, Zoological Survey of India, Kozhikode, Kerala, India

Dr. R. Varatharajan, Manipur University, Imphal, Manipur, India

Dr. Eduard Vives, Museu de Ciències Naturals de Barcelona, Terrassa, Spain

Dr. James Young, Hong Kong Lepidopterists' Society, Hong Kong

Dr. R. Sundararaj, Institute of Wood Science \& Technology, Bengaluru, India

Dr. M. Nithyanandan, Environmental Department, La Ala Al Kuwait Real Estate. Co. K.S.C.,

Kuwait

Dr. Himender Bharti, Punjabi University, Punjab, India

Mr. Purnendu Roy, London, UK

Dr. Saito Motoki, The Butterfly Society of Japan, Tokyo, Japan

Dr. Sanjay Sondhi, TITLI TRUST, Kalpavriksh, Dehradun, India

Dr. Nguyen Thi Phuong Lien, Vietnam Academy of Science and Technology, Hanoi, Vietnam

Dr. Nitin Kulkarni, Tropical Research Institute, Jabalpur, India

Dr. Robin Wen Jiang Ngiam, National Parks Board, Singapore

Dr. Lional Monod, Natural History Museum of Geneva, Genève, Switzerland.

Dr. Asheesh Shivam, Nehru Gram Bharti University, Allahabad, India

Dr. Rosana Moreira da Rocha, Universidade Federal do Paraná, Curitiba, Brasi

Dr. Kurt R. Arnold, North Dakota State University, Saxony, Germany

Dr. James M. Carpenter, American Museum of Natural History, New York, USA

Dr. David M. Claborn, Missouri State University, Springfield, USA

Dr. Kareen Schnabel, Marine Biologist, Wellington, New Zealand

Dr. Amazonas Chagas Júnior, Universidade Federal de Mato Grosso, Cuiabá, Brasil

Mr. Monsoon Jyoti Gogoi, Assam University, Silchar, Assam, India

Dr. Heo Chong Chin, Universiti Teknologi MARA (UiTM), Selangor, Malaysia

Dr. R.J. Shiel, University of Adelaide, SA 5005, Australia

Dr. Siddharth Kulkarni, The George Washington University, Washington, USA

Dr. Priyadarsanan Dharma Rajan, ATREE, Bengaluru, India

Dr. Phil Alderslade, CSIRO Marine And Atmospheric Research, Hobart, Australia

Dr. John E.N. Veron, Coral Reef Research, Townsville, Australia

Dr. Daniel Whitmore, State Museum of Natural History Stuttgart, Rosenstein, Germany.

Dr. Yu-Feng Hsu, National Taiwan Normal University, Taipei City, Taiwan

Dr. Keith V. Wolfe, Antioch, California, USA

Dr. Siddharth Kulkarni, The Hormiga Lab, The George Washington University, Washington,

D.C., USA

Dr. Tomas Ditrich, Faculty of Education, University of South Bohemia in Ceske

Budejovice, Czech Republic

Dr. Mihaly Foldvari, Natural History Museum, University of Oslo, Norway

Dr. V.P. Uniyal, Wildlife Institute of India, Dehradun, Uttarakhand 248001, India

Dr. John T.D. Caleb, Zoological Survey of India, Kolkata, West Bengal, India

Dr. Priyadarsanan Dharma Rajan, Ashoka Trust for Research in Ecology and the Environment

(ATREE), Royal Enclave, Bangalore, Karnataka, India

\section{Fishes}

Dr. Neelesh Dahanukar, IISER, Pune, Maharashtra, India

Dr. Topiltzin Contreras MacBeath, Universidad Autónoma del estado de Morelos, México

Dr. Heok Hee Ng, National University of Singapore, Science Drive, Singapore

Dr. Rajeev Raghavan, St. Albert's College, Kochi, Kerala, India

Dr. Robert D. Sluka, Chiltern Gateway Project, A Rocha UK, Southall, Middlesex, UK

Dr. E. Vivekanandan, Central Marine Fisheries Research Institute, Chennai, India

Dr. Davor Zanella, University of Zagreb, Zagreb, Croatia

Dr. A. Biju Kumar, University of Kerala, Thiruvananthapuram, Kerala, India

Dr. Akhilesh K.V., ICAR-Central Marine Fisheries Research Institute, Mumbai Research

Centre, Mumbai, Maharashtra, India

Dr. J.A. Johnson, Wildlife Institute of India, Dehradun, Uttarakhand, India

Amphibians

Dr. Sushil K. Dutta, Indian Institute of Science, Bengaluru, Karnataka, India

Dr. Annemarie Ohler, Muséum national d'Histoire naturelle, Paris, France

\section{Reptiles}

Dr. Gernot Vogel, Heidelberg, Germany

Dr. Raju Vyas, Vadodara, Gujarat, India

Dr. Pritpal S. Soorae, Environment Agency, Abu Dubai, UAE.

Prof. Dr. Wayne J. Fuller, Near East University, Mersin, Turkey

Prof. Chandrashekher U. Rivonker, Goa University, Taleigao Plateau, Goa. India

Dr. S.R. Ganesh, Chennai Snake Park, Chennai, Tamil Nadu, India

Dr. Himansu Sekhar Das, Terrestrial \& Marine Biodiversity, Abu Dhabi, UAE
Birds

Dr. Hem Sagar Baral, Charles Sturt University, NSW Australia

Dr. Chris Bowden, Royal Society for the Protection of Birds, Sandy, UK

Dr. Priya Davidar, Pondicherry University, Kalapet, Puducherry, India

Dr. J.W. Duckworth, IUCN SSC, Bath, UK

Dr. Rajah Jayapal, SACON, Coimbatore, Tamil Nadu, India

Dr. Rajiv S. Kalsi, M.L.N. College, Yamuna Nagar, Haryana, India

Dr. V. Santharam, Rishi Valley Education Centre, Chittoor Dt., Andhra Pradesh, India

Dr. S. Balachandran, Bombay Natural History Society, Mumbai, India

Mr. J. Praveen, Bengaluru, India

Dr. C. Srinivasulu, Osmania University, Hyderabad, India

Dr. K.S. Gopi Sundar, International Crane Foundation, Baraboo, USA

Dr. Gombobaatar Sundev, Professor of Ornithology, Ulaanbaatar, Mongolia

Prof. Reuven Yosef, International Birding \& Research Centre, Eilat, Israel

Dr. Taej Mundkur, Wetlands International, Wageningen, The Netherlands

Dr. Carol Inskipp, Bishop Auckland Co., Durham, UK

Dr. Tim Inskipp, Bishop Auckland Co, Durham, UK

Dr. V. Gokula, National College, Tiruchirappalli, Tamil Nadu, India

Dr. Arkady Lelej, Russian Academy of Sciences, Vladivostok, Russia

Dr. Simon Dowell, Science Director, Chester Zoo, UK

Dr. Mário Gabriel Santiago dos Santos, Universidade de Trás-os-Montes e Alto Douro,

Quinta de Prados, Vila Real, Portugal

Dr. Grant Connette, Smithsonian Institution, Royal, VA, USA

Dr. M. Zafar-ul Islam, Prince Saud Al Faisal Wildlife Research Center, Taif, Saudi Arabia

Mammals

Dr. Giovanni Amori, CNR - Institute of Ecosystem Studies, Rome, Italy

Dr. Anwaruddin Chowdhury, Guwahati, India

Dr. David Mallon, Zoological Society of London, UK

Dr. Shomita Mukherjee, SACON, Coimbatore, Tamil Nadu, India

Dr. Angie Appel, Wild Cat Network, Germany

Dr. P.O. Nameer, Kerala Agricultural University, Thrissur, Kerala, India

Dr. Ian Redmond, UNEP Convention on Migratory Species, Lansdown, UK

Dr. Heidi S. Riddle, Riddle's Elephant and Wildlife Sanctuary, Arkansas, USA

Dr. Karin Schwartz, George Mason University, Fairfax, Virginia.

Dr. Lala A.K. Singh, Bhubaneswar, Orissa, India

Dr. Mewa Singh, Mysore University, Mysore, India

Dr. Paul Racey, University of Exeter, Devon, UK

Dr. Honnavalli N. Kumara, SACON, Anaikatty P.O., Coimbatore, Tamil Nadu, India

Dr. Nishith Dharaiya, HNG University, Patan, Gujarat, India

Dr. Spartaco Gippoliti, Socio Onorario Società Italiana per la Storia della Fauna "Giuseppe

Altobello", Rome, Italy

Dr. Justus Joshua, Green Future Foundation, Tiruchirapalli, Tamil Nadu, India

Dr. H. Raghuram, The American College, Madurai, Tamil Nadu, India

Dr. Paul Bates, Harison Institute, Kent, UK

Dr. Jim Sanderson, Small Wild Cat Conservation Foundation, Hartford, USA

Dr. Dan Challender, University of Kent, Canterbury, UK

Dr. David Mallon, Manchester Metropolitan University, Derbyshire, UK

Dr. Brian L. Cypher, California State University-Stanislaus, Bakersfield, CA

Dr. S.S. Talmale, Zoological Survey of India, Pune, Maharashtra, India

Prof. Karan Bahadur Shah, Budhanilakantha Municipality, Kathmandu, Nepal

Dr. Susan Cheyne, Borneo Nature Foundation International, Palangkaraja, Indonesia

Dr. Hemanta Kafley, Wildlife Sciences, Tarleton State University, Texas, USA

\section{Other Disciplines}

Dr. Aniruddha Belsare, Columbia MO 65203, USA (Veterinary)

Dr. Mandar S. Paingankar, University of Pune, Pune, Maharashtra, India (Molecular)

Dr. Jack Tordoff, Critical Ecosystem Partnership Fund, Arlington, USA (Communities)

Dr. Ulrike Streicher, University of Oregon, Eugene, USA (Veterinary)

Dr. Hari Balasubramanian, EcoAdvisors, Nova Scotia, Canada (Communities)

Dr. Rayanna Hellem Santos Bezerra, Universidade Federal de Sergipe, São Cristóvão, Brazil

Dr. Jamie R. Wood, Landcare Research, Canterbury, New Zealand

Dr. Wendy Collinson-Jonker, Endangered Wildlife Trust, Gauteng, South Africa

Dr. Rajeshkumar G. Jani, Anand Agricultural University, Anand, Gujarat, India

Dr. O.N. Tiwari, Senior Scientist, ICAR-Indian Agricultural Research Institute (IARI), New

Delhi, India

Dr. L.D. Singla, Guru Angad Dev Veterinary and Animal Sciences University, Ludhiana, India

Dr. Rupika S. Rajakaruna, University of Peradeniya, Peradeniya, Sri Lanka

Dr. Bahar Baviskar, Wild-CER, Nagpur, Maharashtra 440013, India

Reviewers 2018-2020

Due to pausity of space, the list of reviewers for $2018-2020$ is available online.

The opinions expressed by the authors do not reflect the views of the Journal of Threatened Taxa, Wildlife Information Liaison Development Society, Zoo Outreach Organization, or any of the partners. The journal, the publisher, the host, and the partners are not responsible for the accuracy of the political boundaries shown in the maps by the authors.

Journal of Threatened Taxa is indexed/abstracted in Bibliography of Systematic Mycology, Biological Abstracts, BIOSIS Previews, CAB Abstracts, EBSCO, Google Scholar, Index Copernicus, Index Fungorum, JournalSeek, National Academy of Agricultural Sciences, NewJour, OCLC WorldCat, SCOPUS, Stanford University Libraries, Virtual Library of Biology, Zoological Records.

NAAS rating (India) 5.64
Print copies of the Journal are available at cost. Write to:

The Managing Editor, JoTT,

c/o Wildlife Information Liaison Development Society,

No. 12, Thiruvannamalai Nagar, Saravanampatti - Kalapatti Road,

Saravanampatti, Coimbatore, Tamil Nadu 641035, India

ravi@threatenedtaxa.org 


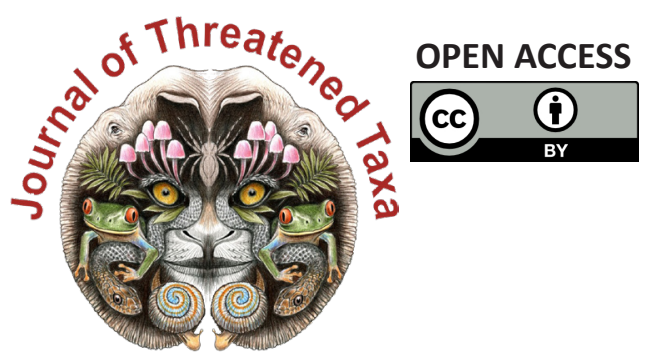

www.threatenedtaxa.org

The Journal of Threatened Taxa (JoTT) is dedicated to building evidence for conservation globally by publishing peer-reviewed articles online every month at a reasonably rapid rate at www.threatenedtaxa.org. All articles published in JoTT are registered under Creative Commons Attribution 4.0 International License unless otherwise mentioned. JoTT allows allows unrestricted use, reproduction, and distribution of articles in any medium by providing adequate credit to the author(s) and the source of publication.

ISSN 0974-7907 (Online) I ISSN $0974-7893$ (Print)

\section{November 2021 | Vol. 13 | No. 13 | Pages: 19887-20142 \\ Date of Publication: 26 November 2021 (Online \& Print) DOI: 10.11609/jott.2021.13.13.19887-20142}

\section{Article}

An inventory of geometrid moths (Lepidoptera: Geometroidea: Geometridae) of KalakadMundanthurai Tiger Reserve, India

- Geetha Iyer, Dieter Stüning \& Sanjay Sondhi, Pp. 19887-19920

\section{Communications}

Roadkills of Lowland Tapir Tapirus terrestris (Mammalia: Perissodactyla: Tapiridae) in one of its last refuges in the Atlantic Forest

- Aureo Banhos, Andressa Gatti, Marcelo Renan de Deus Santos, Leonardo Merçon,

Ilka Westermeyer, Natália Carneiro Ardente, Luis Francisco Oliveira Pereira Gonzaga, Lucas Mendes Barreto, Lucas Damásio, Tomas Lima Rocha, Vitor Roberto Schettino, Renata Valls, Helena Godoy Bergallo, Marcos Vinicius Freitas Silva, Athelson Stefanon Bittencourt, Danielle de Oliveira Moreira \& Ana Carolina Srbek-Araujo, Pp. 19921-19929

Scientific contributions and learning experiences of citizen volunteers with a small cat project in Sanjay Gandhi National Park, Mumbai, India

- Shomita Mukherjee, R. Nandini, P.V. Karunakaran \& Nayan Khanolkar, Pp. 19930-19936

Seasonal food preferences and group activity pattern of Blackbuck Antilope cervicapra (L., 1758) (Mammalia: Cetartiodactyla: Bovidae) in a semi-arid region of western Haryana, India

- Vikram Delu, Dharambir Singh, Sumit Dookia, Priya \& Kiran, Pp. 19937-19947

Studies on the habitats of Grey Francolin Francolinus pondicerianus (J.F. Gmelin, 1789) (Galliformes: Phasianidae) in northern districts of Tamil Nadu, India

- M. Pandian, Pp. 19948-19955

Recovery of vulture population in roosting and scavenging areas of Bastar and Bijapur, Chhattisgarh, India

- Sushil Kumar Dutta, Muntaz Khan, P.R.S. Nagi, Santosh Durgam \& Surabhi Dutta, Pp. 19956-19963

A geographical assessment of Chariganga and Arpara Beel (wetlands) of Nadia, West Bengal as a habitat of wetland birds

- Mehedi Hasan Mandal, Arindam Roy \& Giyasuddin Siddique, Pp. 19964-19975

Phenotypic plasticity in Barilius vagra (Hamilton, 1822) (Teleostei: Danionidae) from two geographically distinct river basins of Indian Himalaya

- Sumit Kumar, Sharali Sharma \& Deepak Singh, Pp. 19976-19984

Taxonomic notes, a new species, and a key to Indian species of the click beetle genus Cryptalaus Ôhira, 1967 (Coleoptera: Elateridae: Agrypninae)

- Harshad Parekar \& Amol Patwardhan, Pp. 19985-19999

Niche overlap of benthic macrofauna in a tropical estuary: diurnal variation

- Mário Herculano de Oliveira, Lidiane Gomes de Lima, Caroline Stefani da Silva Lima, Jéssica de Oliveira Lima Gomes, Franciely Ferreira Paiva, Graciele de Barros, Carlinda Railly Medeiros \& Joseline Molozzi, Pp. 20000-20010

Diversity of aquatic insects and biomonitoring of water quality in the upper Ganga River, a Ramsar site: a preliminary assessment

- Kritish De, Arkojyoti Sarkar, Kritika Singh, Virendra Prasad Uniyal, Jeyaraj Antony Johnson \& Syed Ainul Hussain, Pp. 20011-20018

Patterns of forest cover loss in the terrestrial Key Biodiversity Areas in the Philippines: critical habitat conservation priorities

- Bernard Peter O. Daipan, Pp. 20019-20032

The woody flora of Shettihalli Wildlife Sanctuary, central Western Ghats of Karnataka, India - A checklist

- Kanda Naveen Babu, Kurian Ayushi, Vincy K. Wilson, Narayanan Ayyappan \&

Narayanaswamy Parthasarathy, Pp. 20033-20055

Reproductive biology of Ophiorrhiza caudata C.E.C.Fisch. (Rubiaceae), an endemic and endangered creeping perennial herb of the Western Ghats, India

- Maria Theresa, Appukuttan Kamalabai Sreekala \& Jayalakshmi Mohanlal, Pp. 20056-20065
Short Communications

Successful rescue, medical management, rehabilitation, and translocation of a Red Panda Ailurus fulgens (Mammalia: Carnivora: Ailuridae) in Arunachal Pradesh, India - Jahan Ahmed, Sorang Tadap, Millo Tasser, Koj Rinya, Nekibuddin Ahmed \& Sunil Kyarong, Pp. 20066-20071

A rare photographic record of Eurasian Otter Lutra lutra with a note on its habitat from the Bhagirathi Basin, western Himalaya, India

- Ranjana Pal, Aashna Sharma, Vineet Kumar Dubey, Tapajit Bhattacharya, Jeyaraj Antony Johnson, Kuppusamy Sivakumar \& Sambandam Sathyakumar, Pp. 20072-20077

The first record of Medog Gliding Frog Rhacophorus translineatus Wu, 1977 (Anura: Rhacophoridae) from Chhukha District, Bhutan

- Sonam Lhendup \& Bal Krishna Koirala, Pp. 20078-20083

First record of a freshwater crab, Maydelliathelphusa masoniana (Henderson, 1893) (Decapoda: Brachyura: Gecarcinucidae) from West Bengal, India

- Ram Krishna Das, Pp. 20084-20089

Butterflies of Amrabad Tiger Reserve, Telangana, India

- Deepa Jaiswal, B. Bharath, M. Karuthapandi, Shrikant Jadhav, S. Prabakaran \& S. Rehanuma Sulthana, Pp. 20090-20097

An enumeration of the flowering plants of Kyongnosla Alpine Sanctuary in eastern Sikkim, India

- Sudhansu Sekhar Dash, Subhajit Lahiri \& Ashiho Asoshii Mao, Pp. 20098-20117

A new record of psychrotrophic Paecilomyces formosus (Eurotiales: Ascomycota) from India: morphological and molecular characterization

- Skarma Nonzom \& Geeta Sumbali, Pp. 20118-20123

Notes

Study on incidence and pathology of gastrointestinal parasitic infections in Nilgai Boselaphus tragocamelus in Hisar, Haryana, India

- Maneesh Sharma, B.L. Jangir, D. Lather, G.A. Chandratre, V. Nehra, K.K. Jakhar \& G. Narang, Pp. 20124-20127

An unusual vocalization of Brown Hawk-Owl Ninox scutulata (Raffles, 1822) (Aves:

Strigiformes: Strigidae) recorded from Kerala, India

- Riju P. Nair \& Shine Raj Tholkudiyil, Pp. 20128-20129

New distribution data on the genus Maripanthus Maddison, 2020 (Araneae: Salticidae) from southern India

- A. Asima, John T.D. Caleb, Dhruv A. Prajapati \& G. Prasad, Pp. 20130-20132

On the IUCN status of Boesenbergia albolutea and B. rubrolutea (Zingiberaceae) and typification of $B$. rubrolutea

- K. Aishwarya \& M. Sabu, Pp. 20133-20135

New records of mass seeding Cephalostachyum latifolium Munro (Poaceae) along the midelevation broadleaved forest of Sarpang district, Bhutan

- Jigme Tenzin, Sangay Nidup \& Dago Dorji, Pp. 20136-20139

Response

If habitat heterogeneity is effective for conservation of butterflies in urban landscapes of Delhi, India?' Unethical publication based on data manipulation

- Sanjay Keshari Das \& Rita Singh, Pp. 20140-20142

Publisher \& Host
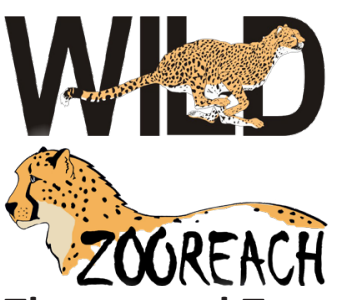

Threatened Taxa 